\title{
Computer simulation and analysis of self-assembled alkylthiol monolayers on the surface of liquid mercury
}

\author{
Dissertation \\ zur Erlangung des mathematisch-naturwissenschaftlichen Doktorgrades \\ "Doctor rerum naturalium" \\ der Georg-August-Universität Göttingen \\ im Promotionsprogramm ProPhys \\ der Georg-August University School of Science (GAUSS)
}

vorgelegt von

Anton Iakovlev

aus Charkiw, Ukraine

Göttingen, 2016 


\section{Betreuungsausschuss}

Prof. Dr. Marcus Müller, Institut für Theoretische Physik, Georg-August-Universität Göttingen

Prof. Dr. Tim Salditt, Institut für Röntgenphysik, Georg-August-Universität Göttingen

Mitglieder der Prüfungskommission

Referent: Prof. Dr. Marcus Müller, Institut für Theoretische Physik, Georg-AugustUniversität Göttingen

Korreferent: Prof. Dr. Tim Salditt, Institut für Röntgenphysik, Georg-August-Universität Göttingen

Weitere Mitglieder der Prüfungskommission:

Prof. Dr. Peter Blöchl, Fakultät für Physik, Georg-August-Universität Göttingen

Prof. Dr. Andreas Tilgner, Institut für Geophysik, Georg-August-Universität Göttingen Dr. H. Jelger Risselada, Institut für Organische und Biomolekulare Chemie, GeorgAugust-Universität Göttingen

Prof. Dr. Dmitry Bedrov, Department of Materials Science and Engineering, University of Utah, USA

Tag der mündlichen Prüfung: 22.07.2016 


\section{Acknowledgments}

I am deeply grateful to my supervisor, Prof. Dr. Marcus Müller, for his indispensable support on my way to the graduation. His keen sense of fundamental physical processes has constantly guided me and made possible to realize this work. I would also like to expresses my gratitude to Prof. Dr. Dmitry Bedrov from the University of Utah (USA) for close and fruitful collaboration as well as for providing useful insights into the self-assembly of alkyl-based surfactants on liquid mercury, which have substantially accelerated the completion of the current work. Moreover, the useful and stimulating discussions with my colleagues from the Technion (Israel), Boris Haimov and Boaz Pokroy, who performed the optical tensiometry experiments, are greatly acknowledged.

I also thank all the members of our research group for their support and time spent together in Göttingen.

The financial support from the Volkswagen Foundation within the joint German-Israeli program under the Grant VW-ZN2726 is highly acknowledged. The computational resources from the GWDG Göttingen, the HLRN Hannover/Berlin, and the JSC Jülich, Germany are gratefully acknowledged as well.

I devote this work to my parents, whose love and care have been always accompanying me. 


\section{Abstract}

In this dissertation I investigate the structure and thermodynamics of alkylthiol (thiol) surfactants on the surface of liquid mercury $(\mathrm{Hg})$ by means of the large-scale Molecular Dynamics (MD) techniques. Simulations in the canonical as well as in the constant temperature-constant stress ensembles are employed in order to comprehensively study these systems at various conditions. Mercury is treated atomistically, whereas the alkylthiol surfactants are modeled within the united-atom formalism, in which alkyl and methyl groups are represented as separate superatoms. The most of results on the self-assembly are presented for octadecanethiol and dodecanethiol systems on liquid mercury. Special care is devoted to the choice of an appropriate model for the modeling of the surface of liquid mercury, because of its unique properties, such as strong surface layering and very high surface tension as for a liquid at room temperatures

I start by analyzing the latest most successful models of liquid mercury in their ability to reproduce the above properties of liquid mercury surface. For this purpose I use classical MD simulations in the canonical and isothermal-isobaric ensembles as well as Monte Carlo techniques as an auxiliary tool. Specifically, atomistic models of liquid mercury such as (i) the density-independent Raabe model, (ii) the density-independent double-exponent model, (iii) the ad-hoc density-dependent double-exponent model, and (iv) two modifications of the embedded-atom models, were compared with each other. Additionally, the liquid-state theory is used to rationalize the phase behavior of these models. Based on the detailed evaluation of the above models I propose an optimized density-independent force field for liquid mercury, which yields the description of the surface of liquid mercury, which is comparable to the generally more accurate embedded-atom models. At the same time my optimized force field for liquid $\mathrm{Hg}$ allows to gain substantial savings in computational time in comparison to the these models.

In the following, I investigate the surface coverage-driven self-assembly of alkylthiols on liquid mercury by the MD simulations in the canonical ensemble. Two co- 
existence regions are found, namely, (i) the coexistence of the agglomerated alkylthiols laying flat (laying-down) with their tails on mercury with the patches of the bare $\mathrm{Hg}$ surface, and (ii) the coexistence of the laying-down thiols with the highly-ordered crystalline islands of the standing-up alkylthiols. It is shown that the crystalline phase is not formed immediately as the full coverage of the laying-down molecules is achieved (as one would expect from the microscopic picture), but rather the monolayer of the oversaturated laying-down molecules continues to exist up to a specific threshold value of the surface coverage. This finding perfectly agrees with the general predictions of the condensation/evaporation theory for finite systems. The deviations of the surface tension from a plateau level in the coexistence region between the laying-down and crystalline alkylthiol phases can be well explained by the corrections due to the line tension of the boundary between the two phases derived in the simplest approximation to this theory. The structure of the crystalline thiolate islands on liquid mercury is characterized in detail and the oblique packing of the alkylthiol headgroups is found.

Finally, the influence of such factors as temperature, surfactant morphology, alkylthiol tail length, lateral pressure and the alkylthiol packings in the crystalline phase higher than that found at coexistence of the crystalline islands with the laying-down phase is investigated. The MD simulations of the temperature and morphology effects are performed in the canonical ensemble for the crystalline alkylthiol islands at coexistence with the respective laying-down phases. The temperature, at which the crystalline island of alkylthiols on liquid mercury "melts", is estimated. By comparing different types of alkylthiol binding to $\mathrm{Hg}$ atoms it is found that the crystalline phase of alkylthiols, in which a single surfactant is attached to a single mercury atom, features a higher level of disorder compared to the respective phases of alkylthiol, when two surfactants can bind to the same mercury atom. To study the effects of different tail lengths, lateral pressure and alkyl thiol packing on the crystalline phases MD simulations in the constant temperature-constant stress ensemble are utilized. This approach allows to avoid the effect of the finite boundaries of the crystalline thiolate islands and to obtain "purified" estimates of their structure. The simulation of the alkylthiols of various lengths reveal no substantial differences. The simulations under lateral compression indicate that the crystalline alkylthiol monolayers on liquid $\mathrm{Hg}$ are more stable upon increase of the lateral pressure compared to Langmuir monolayers on water. My simulations also point out that thiol monolayers undergo a tilting transition upon increase of the lateral packing in the crystalline phases of the standing-up thiols. 


\section{Contents}

1 Introduction 1

1.1 Self-assembly in nature and science . . . . . . . . . . . . . . 1

1.2 Self-assembly on liquid mercury $\ldots \ldots \ldots$. . . . . . . . . . . . . . . . . . . . . 6

1.3 Outline . . . . . . . . . . . . . . . . . . . 13

$\begin{array}{lll}2 \text { Methods } & 15\end{array}$

2.1 Molecular Dynamics . . . . . . . . . . . . . . . . . 15

$2.1 .1 \quad$ Velocity-Verlet integrator . . . . . . . . . . . . . . 15

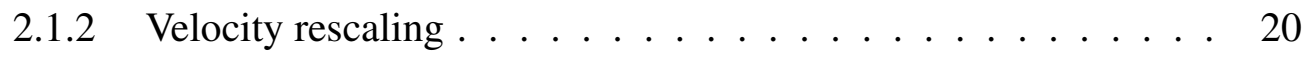

2.1 .3 Berendsen thermo- and barostats . . . . . . . . . . . . . . . . 21

2.1.4 Extended-phase-space approach for simulations in canonical $(N V T)$ and constant stress $(\mathcal{P})$-temperature $(N \mathcal{P} T)$ ensembles $\ldots . \quad 22$

2.1 .5 Multiple time-step scheme . . . . . . . . . . . . . . . . 24

2.2 Monte Carlo simulation . . . . . . . . . . . . . . . . . . . 25

2.3 Liquid-state theory . . . . . . . . . . . . . . . . . . 27

\begin{tabular}{|lll}
3 & Simulation of the surface of liquid mercury & 30
\end{tabular}

3.1 Models of liquid mercury in the literature and the need for a new model 32

3.1 .1 Lennard-Jones model . . . . . . . . . . . . . . . . 32

3.1 .2 Density-independent double-exponent model . . . . . . . . . 33

3.1 .3 Density-dependent double-exponent model . . . . . . . . . . 33

3.1 .4 Raabe model . . . . . . . . . . . . . . . . . . . 36

3.1 .5 Embedded-atom models . . . . . . . . . . . . . . . . . 36

3.1 .6 Simulation details $\ldots \ldots \ldots$. . . . . . . . . . . . . . . . 39

3.1 .7 Results . . . . . . . . . . . . . . . . . . . 40

$3.2 \quad$ Optimized model for liquid mercury $\ldots \ldots \ldots \ldots$. . . . . . . 44

3.2 .1 Derivation . . . . . . . . . . . . . . . . . . . . . . 44

3.2 .2 Validation . . . . . . . . . . . . . . . . . . 47 
3.3 Discussions and conclusions $\ldots \ldots \ldots \ldots$

4 Molecular coverage induced self-assembly of thiols on liquid mercury 51

4.1 Simulation details . . . . . . . . . . . . . . . 52

4.1 .1 Force fields . . . . . . . . . . . . . . . . . 52

4.1.2 Setup of molecular systems and equilibration: Preliminary insights into the self-assembly of thiols on liquid $\mathrm{Hg} \ldots \ldots 58$

4.1 .3 Computational efficiency of LAMMPS . . . . . . . . . . 65

4.2 Coexistence of various alkylthiol phases on mercury . . . . . . . . . . . . . . 66

$4.2 .1 \quad$ Laying-down vs. standing-up phases . . . . . . . . . . . . . 66

4.2 .2 Density profiles . . . . . . . . . . . . . . 70

$4.2 .3 \quad$ Surface density distributions . . . . . . . . . . . . . 72

4.2 .4 Surface tension-surface coverage $(\gamma-\sigma)$ isotherms . . . . . . 72

4.3 Structure in the islands of standing-up thiols . . . . . . . . . . . . 77

4.3 .1 Molecular orientations . . . . . . . . . . . . . . 77

4.3 .2 Packing of the headgroups . . . . . . . . . . . . . 81

4.4 Discussions and conclusions $\ldots \ldots \ldots$. . . . . . . . . . 83

5 In-depth study of densely packed alkylthiol phases on liquid mercury $\quad 86$

5.1 Simulation details . . . . . . . . . . . . . . . . . 87

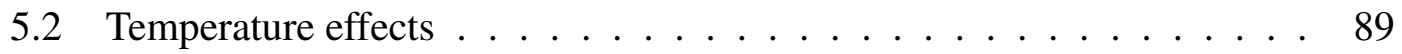

5.3 Influence of molecular morphology: case of the surfactants without S$\mathrm{Hg}-\mathrm{S}$ bond $\ldots \ldots \ldots \ldots \ldots 1 \ldots \ldots \ldots \ldots$

5.4 Chain length effects $\ldots \ldots \ldots$. . . . . . . . . . . . . 96

5.5 Pressure effects . . . . . . . . . . . . . . . . . . . 98

5.6 Effects of surface coverage in the dense SAM and influence of the interaction strength between bound mercury atoms . . . . . . . . . 99

5.7 Conclusions and Discussions $\ldots \ldots \ldots$. . . . . . . . . . . 102

$\begin{array}{lll}6 & \text { Final remarks and outlook } & 104\end{array}$

\begin{tabular}{|lll}
\hline A & Lists of systems simulated in Chap. 4 & 109
\end{tabular}

\begin{tabular}{ll}
\hline Bibliography & 113
\end{tabular}

\begin{tabular}{ll}
\hline List of publications & 125
\end{tabular} 


\section{1 | Introduction}

\subsection{Self-assembly in nature and science}

According to John A. Pelesko, "self-assembly refers to the spontaneous formation of organized structures through a stochastic process that involves pre-existing components, is reversible, and can be controlled by proper design of the components, the environment, and the driving force" [1]. This is probably one of the most general definitions of the self-assembly. Self-assembly processes are ubiquitous in nature and one can find results of their action on all length and interaction scales, e.g. in the form of seashells or spiral galaxies. Numerous illustrations of self-assembly are also present in abundance in various solutions (micellization), colloids, molecular bilayers, liquid crystals, emulsions, as well as in all sorts of biological systems [2]. The scope of this work is devoted to the molecular self-assembly at interfaces.

The classical example of self-assembled structures at interfaces are Langmuir monolayers of amphiphilic molecules at the interface of water. An example of such a monolayer is depicted in Fig. 1.1 for the case of palmitic acid $\left(15\left(\mathrm{CH}_{2}\right) \mathrm{COOH}\right)$ on water [3]. A molecule constituting such a monolayer consists of a polar hydrophilic headgroup and of a hydrophobic, often neutral, tail. The hydrophilic headgroup is anchored on water via electrostatic interactions, whereas the hydrophobic tails stay tilted at $\sim 5^{\circ}-29^{\circ}[3]$ to the surface normal on water and interact via the Van der Waals forces among themselves. This intermolecular interaction between the tails is responsible for the long-range order in the Langmuir films. As a rule the long-range order in a Langmuir self-assembled film increases as the surfactant surface coverage, $\sigma$, (or its inverse, the area per surfactant) increases (decreases). During this process the monolayer undergoes various phase transitions ranging from a dilute gas phase via low-ordered liquid phase up to highly- 


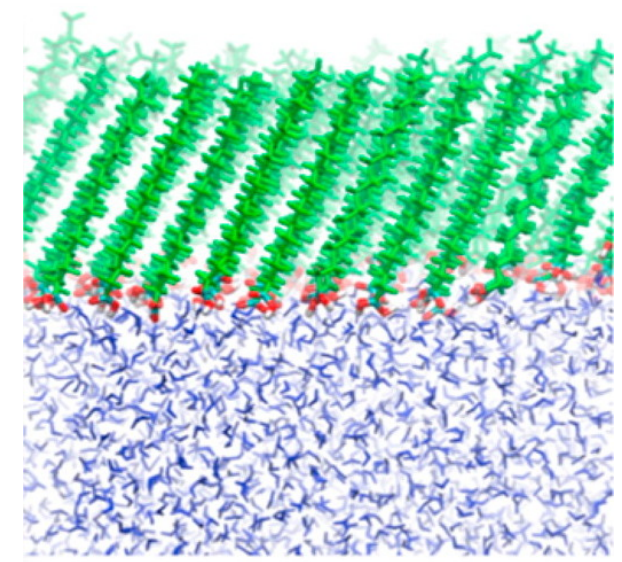

Figure 1.1: A snapshot from a molecular simulation illustrating the Langmuir monolayer of palmitic acid $\left(15\left(\mathrm{CH}_{2}\right) \mathrm{COOH}\right)$ on water for an area per molecule of $22 \AA^{2}$ and a surface pressure of $5.6 \mathrm{mN} / \mathrm{m}$ at $T=300 \mathrm{~K}$. Color code: alkyl tails (green); polar $\mathrm{COOH}$ group, (red); water (blue). Adapted with permission from [W. Lin, A. J. Clark, F. Paesani, Langmuir, 31, 2147 (2015)]. Copyright (2016) American Chemical Society.

ordered condensed amphiphile phases [4]. One can see the signatures of these phase transformations as plateau regions in a surface pressure-area per molecule (or, alternatively, in the surface tension-surface coverage) isotherm, which is sketched in Fig. 1.2 [4]. In this context the surface pressure, $\Pi=\gamma_{0}-\gamma$, means the difference between the surface tension of the bare water, $\gamma_{0}$, and of the water surface with a given number of the surfactants on it, $\gamma$. Usually the surface tension at a given value of $\sigma$ is obtained from tensiometry experiments by measuring the force acting on a suspended plate, which should be completely wetted by the material from the trough (see e.g. [4, 5]). The surfactant surface coverage in such experiments is typically controlled by adjusting the area available to the monolayer via appropriately setting the side barriers in a Langmuir trough (Fig. 1.2 top). Langmuir monolayers at the interfaces with water have been known to mankind over centuries and been a subject of ongoing research (e.g. [3]) since the end of the 18-th century on. A comprehensive review of these systems is given by Kaganer et al. [4].

Another prominent example of organic self-assembled monolayers (SAMs) are alkylthiol (or simply thiol) films on gold $(\mathrm{Au})$ [6-9]. In this case a special ordering of the tails, as well as of the headgroups is epitaxially reinforced by the underlying crystal structure. The headgroups, typically sulfur (S), of the constituent molecules are 


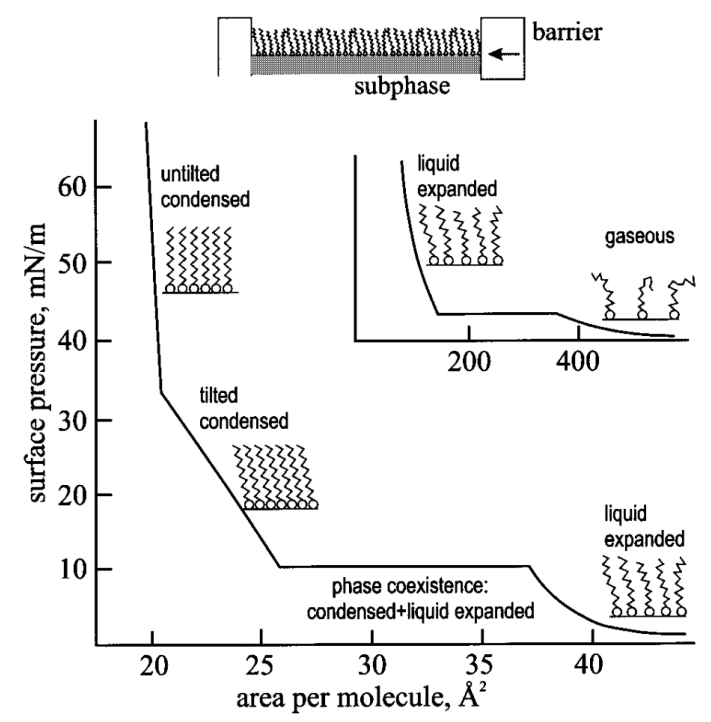

Figure 1.2: A schematic view of a Langmuir trough (top) and a general isotherm of Langmuir monolayer (bottom). Horizontal plateaus in the isotherm correspond to phase coexistence regions at first-order transitions. Reprinted figure with permission from [V. M. Kaganer, H. Mohwald, P. Dutta, Rev. Mod. Phys., 71, 779 (2015).] Copyright (2016) by the American Physical Society.

bound to the Au substrate via covalent bonds. Less frequently selen is used as a headgroup [7, 10]. Alkythiol SAMs on other crystalline substrates such as graphene, gallium arsenide, silver, zinc selenide, copper, germanium etc. have been also experimentally studied [6, 11-16]. Alkylthiol SAMs on gold gained enormous attention from experimental physicists (see e.g. Refs. [6-9, 17- 25]). The alkylthiol SAMs allow to modify surface properties of solid materials in a controlled way, since the thiol endgroups can be easily exchanged [6]. This finds numerous applications in nano- and biotechnology, e.g. for creation of biocompatible surfaces, (bio)sensors, corrosion and wear protection, nanopatterning, controlling of such macroscopic properties as wetting, adhesion, friction etc. [6-8, 26]. Seminal experimental observations were described by Nuzzo et al. [23] and Poirier et al. [25]. The former proposed the $(\sqrt{3} \times \sqrt{3}) R 30^{\circ}$ packing of the sulfur headgroups that is commensurate with the underlying Au (111) surface [23], which is schematically depicted in Fig. 1.3 . The notation $(\sqrt{3} \times \sqrt{3}) R 30^{\circ}$ denotes the hexagonal arrangement of the sulfur headgroups, which is rotated by $30^{\circ}$ relatively to the underlying $\mathrm{Au}$ (111) surface and has the nearest-neighbor distance of $d_{a u} \sqrt{3}$ with 


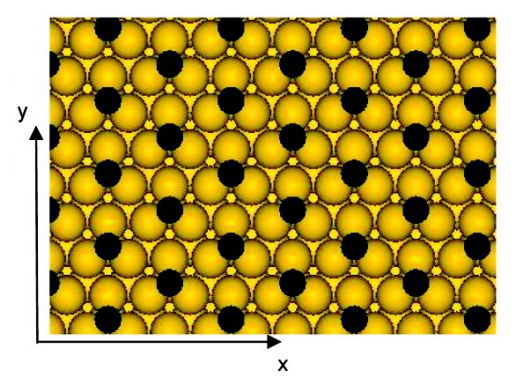

Figure 1.3: Schematic view of the typical $(\sqrt{3} \times \sqrt{3}) R 30^{\circ}$ packing of sulfur headgroups (black) on $\mathrm{Au}$ (111) surface (yellow). The sulfur headgroups are arranged in a hexagonal manner with the nearest-neighbor distance of $d_{a u} \sqrt{3}$, where $d_{a u}=2.88 \AA$ is the nearest-neighbor distance of the hexagonal structure of the gold atoms in the substrate. The unit cell of the sulfur groups is rotated by $30^{\circ}$ with respect to the underlying $\mathrm{Au}$ (111) surface. Adapted with permission from [L. Ramin, A. Jabbarzadeh, Langmuir, 28, 4102 (2012)]. Copyright (2016) American Chemical Society.

$d_{a u}$ being the lattice spacing of Au. Poirier et al. gave the first description of the selfassembly mechanism on $\mathrm{Au}$ (111) [25]. In the case of other crystalline substrates the order of the thiol SAMs has been also found to be commensurate with the one of the underlying crystal lattice [6].

SAMs are usually created by a molecular deposition of thiols from a gas or a liquid phase onto a substrate [6--9, 27]. This process usually involves multiple stages, which are sketched in Fig. 1.4. Here, unlike the Langmuir monolayers, the alkyl tails do not experience strong repulsive interaction with the substrate and adsorb initially onto the metal surface with their tails laying flat on it (Fig. 1.4 a). This stage typically lasts 2-15 min. In the following molecules in such conformations will be called layingdown molecules. The layer of the laying-down thiols is subsequently completed as the surfactant surface coverage, $\sigma$, grows (Fig. $1.4 \mathrm{p}$ ). When $\sigma$ exceeds the value of the

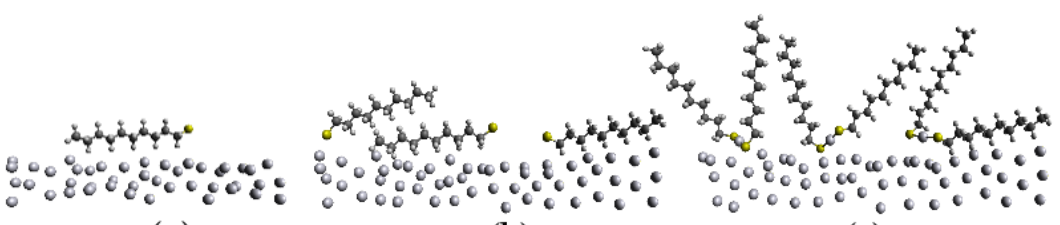

(a) (b)

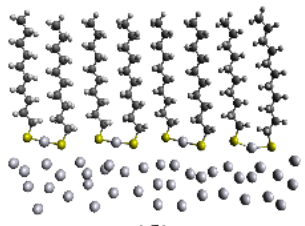

(d)

Figure 1.4: Schematic view of the alkylthiol self-assembly on the surface of liquid mercury. This chart illustrates general stages of alkylthiol self-assembly on metal surfaces. 
full coverage of the laying-down conformations, the backbones of thiols reorient and the nucleation of the standing-up phase is observed (Fig. 1.4k), where thiols stand tilted at a sharp angle to the surface normal. This is followed by several protracted stages of the self-assembly, which are often associated with the increase of ordering within the monolayer of the tilted molecules. The final stages of the self-assembly can last from a couple of hours to several days and result in thiol SAMs that feature long-range crystalline order (Fig. 1.4d). The tilt angle of the SAMs on crystalline substrates is typically $30^{\circ}[6]$.

Computer simulation studies of the self-assembled alkylthiol monolayers on metals [28-46] focus on the SAMs on crystalline surfaces (mostly Au) as well. Moreover, in the vast majority of the simulations only the structure of the densely packed monolayers of thiols standing tilted to the surface normal (i.e. at the final stage of the self-assembly) was probed. Apart from several simplified model systems [47-49], no investigation of molecular self-assembly as a function of the number of alkylthiol surfactants on metallic (liquid) surfaces has been attempted.



Figure 1.5: Potential structure of a dense self-assembled monolayer of alkylthiol surfactants on the surface of liquid mercury at room temperature. Color code: alkyl tails, green; sulfur headgroups, yellow; bulk mercury, red; mercury atoms with thiols adsorbed onto them (bound mercury), purple. 


\subsection{Self-assembly on liquid mercury}

Fig. 1.5 illustrates a potential structure of a dense self-assembled monolayer of alkylthiol surfactants on the surface of liquid mercury at room temperature. The reader can see that the alkyl tails (green) are tilted to the surface normal at a sharp angle. Furthermore, all the molecules display a collective azimuthal tilt direction. The sulfur headgroups (yellow) feature a highly ordered two-dimensional (2D) arrangement, as well as the mercury atoms covalently bound to the sulfurs (purple) do. In the following the latter type of atoms will be called the bound mercury. The whole SAM structure sits on a very dense and strongly stratified, but yet liquid, surface of mercury (red). In Chap. 4 we will see that it is in fact much more intuitive and easier to analyze the unit cell of the bound mercury atoms, than the one of the sulfurs, which will in any case follow the bound mercury.

The seamless, liquid, yet, at the same time, very dense surface of liquid mercury $(\mathrm{Hg})$ makes it possible to create thiol SAMs, which are free of the characteristics of underlying crystal substrates and accompanying defects providing a number of benefits over traditional crystalline materials. These exceptional properties of liquid $\mathrm{Hg}$ make it especially suitable for investigations of charge transfer through organic films, where liquid mercury is typically used in the form of a drop-electrode by being placed on top of the SAM that is preadsorbed on another crystalline electrode [50]. This architecture of a metal- or semiconductor-molecule-metal junction does not deteriorate the structure of the SAM [51]. One could also cover a $\mathrm{Hg}$ droplet electrode with an organic layer prior to putting it into contact with the other SAM on the crystalline electrode. Such a technique is applied in order to inhibit the amalgamation of the crystalline electrode by mercury [52]. All these features serve for the wide usage of thiol SAMs in the rapidly expanding field of organic electronics [50-55].

At the same time very little is known about the properties of the alkylthiol SAMs on liquid mercury. An original and simple idea on how to study the effect of a microscopic thiol self-assembly on a liquid metal was recently proposed by our colleagues, Boris Haimov and Boaz Pokroy, from the Technion (Haifa, Israel) [27]. This idea links the microscopic molecular self-assembly on the liquid $\mathrm{Hg}$ surface with such macroscopic quantity as surface tension, $\gamma$, and is based on the observation that the adsorption of thiols onto a sessile droplet of liquid $\mathrm{Hg}$ results in a pronounced decrease of its sur- 
face tension. If one knows the local pressure difference, $\Delta P$, at the surface of the $\mathrm{Hg}$ droplet in the gravity field and its principal curvature radii, $R_{1}$ and $R_{2}$, it is straightforward to calculate $\gamma$ by using the Young-Laplace equation, $\Delta P=\gamma\left(1 / R_{1}+1 / R_{2}\right)$, where $\Delta P=g z \Delta \rho$ and $\Delta \rho$ being the change of the density across the droplet surface, $g$ is the gravitational constant and $z$ is the height of the chosen point at the surface of the $\mathrm{Hg}$ droplet, and $R_{1}$ and $R_{2}$ are calculated from the shape of the $\mathrm{Hg}$ droplet via image recognition techniques [27]. The particular advantages of this method, which is known as the optical tensiometry, are that it allows to track the effect of the thiol adsorption from a solution (say ethanol) onto a liquid mercury droplet in real time and that it does not rely on contact angle measurements that involve the tension of the liquid and vapor with the supporting substrate. Using this methodology it was shown that thiols of longer tail lengths reduce the surface tension of a mercury droplet in ethanol faster than the ones with shorter tails [27]. At first glance this might seem counterintuitive, since longer molecules are expected to diffuse slower. My explanation of this observation invokes a quite simple argument. One has to distinguish between the rate of adsorption, $k_{\sigma}=d \sigma / d t$, and the rate of reduction of the surface tension, $k_{\gamma}=-d \gamma(\sigma) / d t$. Here $\gamma, \sigma$ and $t$ stand for the surface tension of the $\mathrm{Hg}$ droplet, surface coverage of surfactants on the droplet and time, respectively. In the initial stage of adsorption the adsorbed molecules resemble a dilute two-dimensional (2D) gas of molecules laying flat with their tails on $\mathrm{Hg}$, and the reduction of the surface tension is proportional to the number of adsorbed molecules, $k_{\gamma}=k_{b} T k_{\sigma}$, where $k_{b}$ is the Boltzmann constant. In the experimentally relevant regime, however, lateral interactions between the molecules on the $\mathrm{Hg}$ surface become important, and the surface tension at a particular value of $\sigma$ may be captured by the Volmer isotherm [56]

$$
\gamma=\gamma_{0}-\frac{k_{b} T \sigma}{1-a_{t h} \sigma}
$$

which links the equilibrium value of surface tension with the respective value of the surfactant surface coverage, $\sigma$. In the above equation $\gamma_{0}$ is the value of the surface tension for a surfactant-free surface, and $a_{t h}$ may be interpreted as the excluded area per thiol and plays essentially the role of the second-order virial coefficient. By differentiating the Volmer isotherm with respect to time one obtains the following expression for the 
rate of reduction of the surface tension

$$
k_{\gamma}=\frac{k_{b} T}{\left(1-a_{t h} \sigma\right)^{2}} k_{\sigma} .
$$

The adsorption rate, $k_{\sigma}$, depends on the self-diffusion coefficient, $D$, of the molecule in solution like

$$
k_{\sigma}=\frac{\Delta N}{A \Delta t}=\frac{C_{0} \Delta l}{\Delta t}=\frac{C_{0} \sqrt{2 D \Delta t}}{\Delta t} \sim \sqrt{D}
$$

where $\Delta N$ is the number of molecules that adsorb on the surface of area $A$ in time $\Delta t$, $C_{0}$ is the concentration of the molecules in solution, $\Delta l$ is the diffusion length of the molecules perpendicular to the $\mathrm{Hg}$ surface. $a_{t h}$ scales like the number of monomers (alkyl groups) $n_{m}$, and the self-diffusion coefficient, $D$, scales like $1 / n_{m}$ or like $1 / \sqrt{n_{m}}$ in the Rouse or Zimm model (see e.g. [57]), respectively. Taking this into account and by inserting Eq. 1.3 into Eq. 1.2 we obtain in the least favorable case (Rouse diffusion)

$$
k_{\gamma} \sim \frac{k_{b} T}{n_{m}^{1 / 4}}+2 k_{b} T a_{c h} \sigma n_{m}^{3 / 4},
$$

where $a_{c h}=a_{t h} / n_{m}$ is the surface area per monomer. The above relation provides an order-of-magnitude estimate of how fast the surface tension is reduced by the molecules having different number of monomers (i.e. having different length), and it explains why the rate of reduction of the surface tension increases with the increase of length of the adsorbate molecules, although the diffusive transport becomes slower.

In the adsorption experiments the surface tension, $\gamma$, is measured as a function of adsorption time (see e.g. Fig. 1.6). The optical tensiometry results have also revealed multiple temporal regimes of adsorption of thiols on liquid mercury, namely, a very rapid decrease of the surface tension during the first 2-3 min of adsorption is followed by a much slower subsequent decline of $\gamma$ [27]. This yields evidence, though indirect, of complex structural transformations within the thiol SAM. In order to interpret these findings and to understand the underlying adsorption mechanism one can relate the evolution of $\sigma$ with time through various kinetic models of adsorption (see below) and then, assuming that the corresponding equilibrium values of $\gamma$ at the respective $\sigma(t)$ are given by the Volmer isotherm (Eq. 1.1), try to find out which of the kinetic models most appropriately describes the experimental data. Similar approaches are routinely used in 


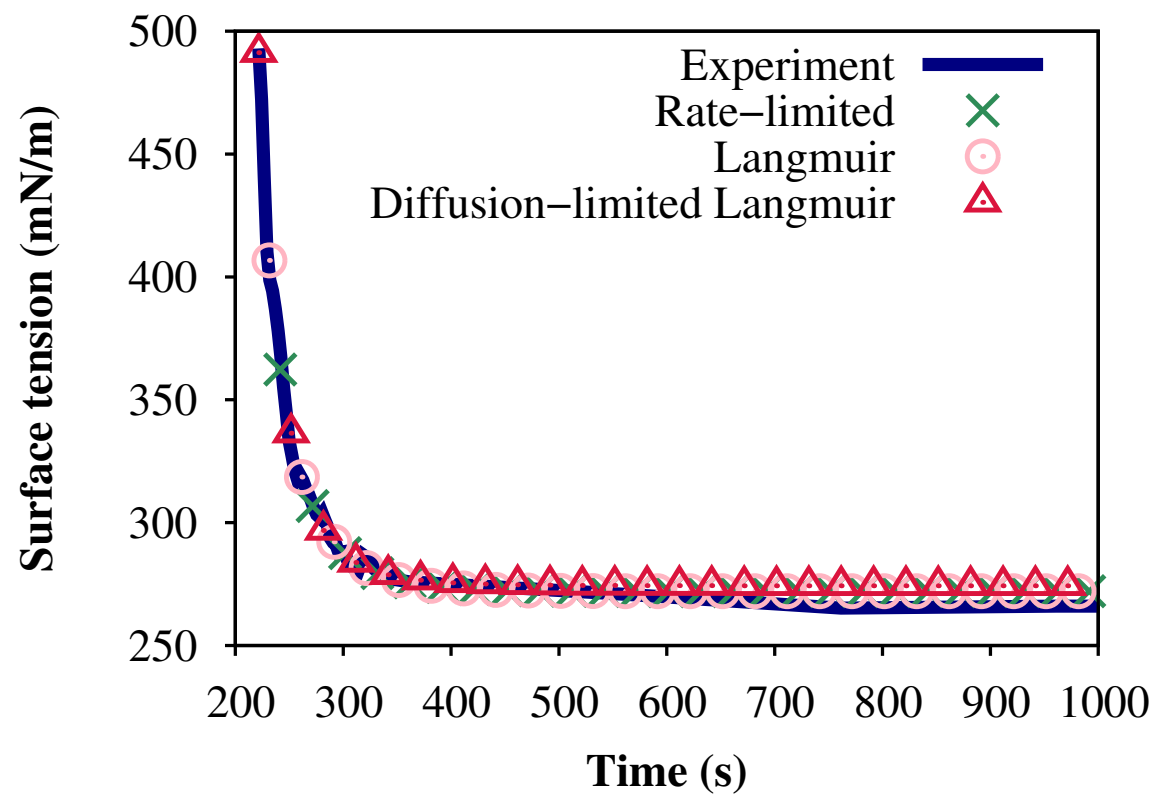

Figure 1.6: Fit of the experimental curve of the surface tension [27] as a function of time with the Volmer equation (Eq. 1.1) under the usage of various adsorption models (Eqs. 1.5 1.7). The plot is shown for the adsorption of dodecanethiols on the liquid mercury droplet in ethanol. The concentration of dodecanethiols in ethanol and temperature were $1 \mathrm{mM}$ and $303 \mathrm{~K}$, respectively.

the studies of Langmuir monolayer [4]. According to the Langmuir model of adsorption the time dependence of $\sigma$ is given by [58]

$$
\sigma(t)=\sigma_{\max }\left(1-e^{-k t}\right)
$$

where $\sigma_{\max }$ is some maximally attainable value of $\sigma$ and $k$ is the rate of adsorption. This model corresponds to a single-stage adsorption process, when a molecule reaching the surface at the rate $k$ becomes immediately adsorbed once it touches the surface. One can perform fitting of the experimental $\gamma(t)$ by the expression obtained by inserting $\sigma(t)$ given by the Langmuir model (Eq. 1.5) into the Volmer isotherm (Eq. 1.1). The resulting fit of the experimental data [27] for the case of dodecanethiol is shown in Fig. 1.6 and the obtained fitting parameters are $k_{b} T \sigma_{\max }=0.036 \mathrm{eV} / \AA^{2}, a_{t h} \sigma_{\max }=$ $-1.45, k=0.023 \mathrm{~s}^{-1}$, which yields $1 / \sigma_{\max }=0.73 \AA^{2}$ and $a_{t h}=-1.05 \AA^{2}$ for $T=$ $303 \mathrm{~K}$ (temperature in the experiment [27]). 
In the case of the diffusive transport of the molecules to the substrate the diffusionlimited Langmuir model describes the change of $\sigma$ with time [58]

$$
\sigma(t)=\sigma_{\max }\left(1-e^{-\tilde{k} \sqrt{t}}\right)
$$

Likewise, by inserting Eq. 1.6 into Eq. 1.1 and performing the fitting of the experimental $\gamma(t)$, one obtains $k_{b} T \sigma_{\text {max }}=0.0021 \mathrm{eV} / \AA^{2}, a_{t h} \sigma_{\text {max }}=0.853, \tilde{k}=0.529 \mathrm{~s}^{-1 / 2}, 1 / \sigma_{\text {max }}=$ $12.23 \AA^{2}$, and $a_{t h}=10.432 \AA^{2}$. The respective fit is shown in Fig. 1.6 . In this case the diffusion coefficient, $D$, of dodecanethiols in ethanol equals to $4.1 \mathrm{~cm}^{2} / \mathrm{s}$, which compares well with typical diffusion constants for thiol systems [27]. This estimate was obtained by using $D=\tilde{k}^{2} \pi \sigma_{\max }^{2} /\left(4 C_{0}\right)$ [59] with $C_{0}=1 \mathrm{mM}$ being the concentration of dodecanethiol in ethanol as used in the experiments [27].

Finally, in a more complex scenario when a molecule can first be adsorbed into a precursor state, from which it can either desorb back into the solution or be irreversibly adsorbed onto the surface, the kinetics is described by the rate-limited model [58]

$$
\sigma(t)=\sigma_{\max }\left(\frac{e^{k^{\prime} t}-1}{e^{k^{\prime} t}+k_{E}}\right)
$$

where $k^{\prime}=\left(1+k_{E}\right) k$ and $k_{E}$ accounts for the adsorption into the precursor state. Similarly, by inserting Eq. 1.7 into Eq. 1.1 and carrying out the fitting of the experimental data one obtains $k_{b} T \sigma_{\max }=0.0068 \mathrm{eV} / \AA^{2}, a_{t h} \sigma_{\max }=0.53, k_{E}=-0.81, k^{\prime}=0.023 \mathrm{~s}^{-1}$, and $1 / \sigma_{\max }=3.82 \AA^{2}, a_{t h}=2.04 \AA^{2}$. The respective fit is shown in Fig. 1.6 .

The usage of any of the above adsorption models fits well the experimental data (Fig. 1.6), but the physically meaningful $\left(1 / \sigma_{\max }>10 \AA^{2}\right)$ fitting parameters were obtained only in the case of the diffusion-limited adsorption model (see also [27]) indicating that the adsorption of thiols onto the $\mathrm{Hg}$ droplet in ethanol occurs through a diffusive transport of thiols towards the surface with a subsequent chemical adsorption onto it. This behavior contrasts to the case of thiol adsorption onto gold, where the rate-limited model (Eq. 1.7) was describing the adsorption process better [58]. My analysis of the optical tensiometry results have been presented alongside the experiments in Ref. [27].

The above adsorption experiments and the respective fits give, of course, only an idea of the self-assembly on liquid mercury for the initial stages and provide no structural data. But what happens at the later stages of the self-assembly, when the thiol sur- 

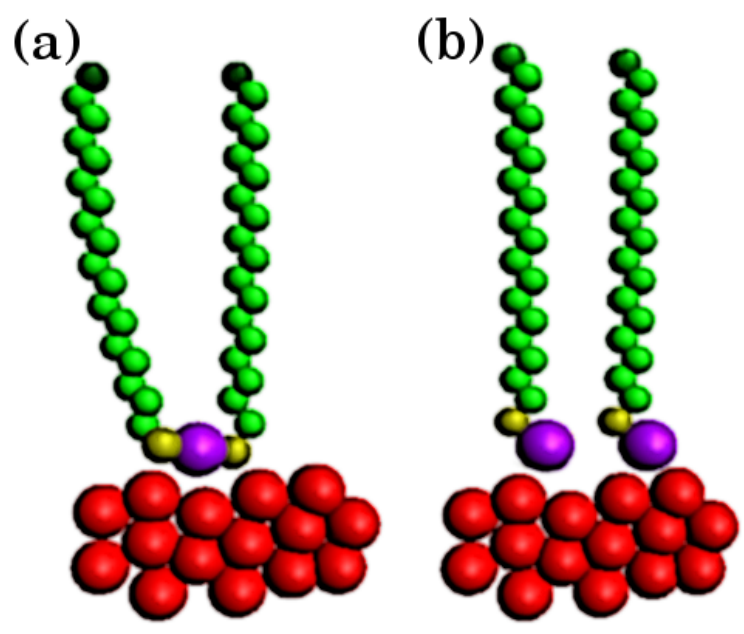

Figure 1.7: Schematic view of possible chemical binding patterns of an alkylthiol surfactant onto the surface of liquid mercury. (a) A surfactant complex created by two alkylthiol molecules and a single $\mathrm{Hg}$ atom $(\mathrm{R}-\mathrm{S}-\mathrm{Hg}-\mathrm{S}-\mathrm{R})$, in which each thiol is attached to a single $\mathrm{Hg}$ atom via a covalent bond between its sulfur headgroup and one of the surface $\mathrm{Hg}$ atoms. The angle between the resulting subsequent $\mathrm{S}-\mathrm{Hg}$ and $\mathrm{Hg}-$ $\mathrm{S}$ bonds ( $\mathrm{S}-\mathrm{Hg}-\mathrm{S}$ bond) is $\sim 180^{\circ}$. (b) A surfactant complex without $\mathrm{S}-\mathrm{Hg}-\mathrm{S}$ bond $(\mathrm{Hg}-\mathrm{S}-\mathrm{R})$. Here a single thiol surfactant is covalently bonded to one of the surface $\mathrm{Hg}$ atoms. Color code: methyl group $\left(\mathrm{CH}_{3}\right)$, dark green; alkyl group $\left(\mathrm{CH}_{2}\right)$, green; sulfur headgroup (S), yellow; a mercury atom incorporated withing the $\mathrm{S}-\mathrm{Hg}-\mathrm{S}$ bond, also denoted as the bound mercury $\left(\mathrm{Hg}^{*}\right)$, purple; bulk mercury $(\mathrm{Hg})$, red. This figure also illustrates the united-atom approach for molecular simulations, where $\mathrm{CH}_{3}$ and $\mathrm{CH}_{2}$ groups are modeled as single "super" atoms, which are described by some effective interactions chosen such that to maximally realistic reproduce the properties of the real substances (see e.g. the OPLS potentials [60])

face coverage is high and more complex structures are formed? What are the possible thiol phases on liquid $\mathrm{Hg}$ and the respective conformational transformations that may cause the long relaxation times seen in the adsorption experiments? Are there, against common believe, defects in the structure of SAMs on the surface of liquid mercury? What is the structure of those phases and defects? Are the thiols already chemisorbed in the low coverage regime, when they lay flat with their tails on the $\mathrm{Hg}$ surface? Which scenario is more realistic: Two thiol molecules form a surfactant complex bound by a single surface $\mathrm{Hg}$ atom (Fig. 1.7a) or a single thiol molecule attaches to a single surface $\mathrm{Hg}$ atom (Fig. 1.7p)? Finally, what are the packings of the headgroups and alkyl tails?

The reported experimental data on the structure and self-assembly of thiol SAMs on 
$\mathrm{Hg}$ is quite scarce and partially contradictive. The first Grazing Incidence Diffraction (GID) X-Ray measurements on this systems by the group of Deutsch et al. did not find evidence of ordered SAMs on $\mathrm{Hg}$ [61]. In the next work [62] the same group using GID techniques predicted an oblique unit cell for the sulfur headgroups with one sulfur per unit cell and the base vectors of very similar lengths; and the results of the previous GID measurements [61] were attributed to the beam damage of the sample [62]. As we shall see in Chap. 5, such a unit cell may in principle exist under the condition that a single thiol surfactant is attached to a one surface mercury atom (Fig. 1.7p). Nevertheless, such scenario seems to be unlikely, because in the prevailing majority of mercuro-organic compounds mercury has a valence of two [63]. In the following publications Deutsch et al. conjectured a rectangular non-centered unit cell with two sulfurs per unit cell [64, 65] indicating that two thiols were attached to a single surface $\mathrm{Hg}$ atom (Fig. 1.7 a). The results of the preceding measurements [62], where a single sulfur per unit cell was proposed, one should probably also ascribe to the effects due to a beam damage. It should be noted that an interpretation of the X-Ray data as an oblique unit cell with two sulfurs could not be excluded [65]. Despite this, the quantitative characteristics of the unit cell have been only estimated under the conjecture of a rectangular packing for a single system of octadecanethiols (SC18) at $T=299 \mathrm{~K}$. Accordingly, the lengths of the base vectors of the unit cell were 5.52 and $8.42 \AA$ yielding the thiol surface coverage of $4.3 \mathrm{~nm}^{-2}$ for the dense SAM of thiols [64, 65]. In addition, tensiometry measurements on thiol SAMs on liquid $\mathrm{Hg}$ indicated coexistence regions of single molecular layers laying parallel to the mercury surface and of standing molecules tilted with respect to the surface normal [64]. Low density 2D gas phase of thiols on liquid $\mathrm{Hg}$ was deduced from the fits by the Volmer isotherm of the data for the thiol surface coverage lower than the one of the first completely filled layer of laying molecules [64]. Fourier-transformed infrared spectroscopy results have also confirmed an increasing ordering in the alkylthiol SAMs on liquid $\mathrm{Hg}$ with the increase in the thiol surface coverage [52], but no interpretation of this data in terms of the molecular packing and respective unit cells has been given.

For the sake of completeness, it should be also mentioned that phase coexistence regions were found for alcohols [66], fatty acid [67], as well as for normal alkanes on the surface of liquid mercury [5]. In the case of alcohols such phases as single, double, triple and quadruple layers of the molecules parallel to the $\mathrm{Hg}$ surface as well as a monolayer 
of molecules standing upright have been detected [66]. This is probably the most rich phase behavior seen on liquid $\mathrm{Hg}$ so far. Fatty acids have a phase diagram similar to that of alcohols with the main difference that no triple or quadruple multilayers of molecules laying flat on the $\mathrm{Hg}$ surface were seen [67]. Finally, normal alkanes exhibit phases of single, double and triple layers of molecules laying on the $\mathrm{Hg}$ surface prior to the formation of the respective alkane bulk phases [5].

\subsection{Outline}

In the present work I make the first step towards understanding the self-assembly of alkylthiols on liquid mercury by large-scale molecular simulations. There has been no previous endeavor to model these systems using modern simulation techniques. The development of computer simulations of organic self-assembled monolayers on liquid mercury appears to be crucial in order to answer the above questions, and to achieve an understanding of the SAM structure and thermodynamics on $\mathrm{Hg}$, as well as to facilitate the future advancement in the areas of application briefly discussed in the previous section. In my thesis I mainly focus on the investigation of the alkylthiol conformation on liquid mercury as a function of the molecular surface coverage. On the way to this final goal one has first to decide to which extent the features of the substrate are important. A naive choice would be to model the liquid mercury as a flat surface as is done, for instance, in some simulations of Langmuir monolayers on water [68, 69] or thiol SAMs on gold [34, 38, 45, 46]. Such choice would inevitably erase all the details of the chemical binding of thiols to mercury. Unfortunately, one cannot also simply hold the positions of the mercury atoms fixed in the simulation (as in the majority of cases for SAMs on $\mathrm{Au}$ ), since this will completely neglect all the SAM relaxations permitted by the liquid nature of mercury at room temperature. This is why my computational model allows to the atoms in the substrate, i.e. the $\mathrm{Hg}$ atoms, to move according to the dynamic equations of motion. As the reader shall see in Chap. 4, the number of $\mathrm{Hg}$ atoms that one has to simulate in this case appears to be exceedingly large, $\sim 3 \times 10^{5}$, compared to several thousands of Au atoms, which were allowed to move in a few computer studies of SAMs on Au [32, 37, 42, 43]. This is necessary in order to account for the pronounced surface layering of liquid mercury (as explained in Chap. 3). Therefore, I devote special attention in my thesis to the computationally efficient simulation 
of the surface of liquid mercury. In the next chapter I will introduce the foundations and principles of the simulation techniques used herein. Then in Chap. 3 the most successful models of liquid mercury suitable for the intended large-scale simulations will be evaluated with respect to their ability to simulate the surface of liquid mercury at room temperatures. Based on the results of this evaluation I will propose an optimized force field for liquid mercury. Afterwards, in Chap. 4 the alkylthiol molecular conformation for various surface coverages will be simulated and analyzed on the example of octa(SC18) and dodecanethiols (SC12). Following, in Chap. 5 the influence of such factors as temperature, molecular morphology, chain length, and lateral pressure on the thiol SAMs on liquid mercury will be studied. In the end I will finish with final remarks and conclusions. 


\section{2 | Methods}

In this chapter the techniques used to simulate and rationalize the obtained results for the bare surface of liquid mercury as well as for the alkylthiol SAMs on it are discussed. In the beginning, in Sec. 2.1, classical Molecular Dynamics methods for the simulations in various statistical ensembles are covered, since they are used through out the thesis as the main tool for the relaxation of the SAMs on $\mathrm{Hg}$ towards equilibrium and studying their respective equilibrium structural and thermodynamic properties. In Sec. 2.2 the Metropolis Monte Carlo method is explained, which is used in Chap. 3 to support the Molecular Dynamics results for some of the investigated models of liquid mercury. Finally, in Sec. 2.3 the liquid-state theory is described, which appears to be especially convenient for understanding the equation of state of the atomistic models of liquid mercury with density-dependent interactions.

\subsection{Molecular Dynamics}

\subsubsection{Velocity-Verlet integrator}

The essence of classical Molecular Dynamics (MD) simulation techniques consists in a numerical solution of the classical Hamiltonian equations of motion for $N$ particles that represent a system under investigation (see e.g. [70]). When the system is subjected to no constraints, these equations result in the system of $6 \mathrm{~N}$ coupled equations

$$
\begin{gathered}
\dot{\mathbf{r}}_{i}=\frac{\partial H}{\partial \mathbf{p}_{i}}=\frac{\mathbf{p}_{i}}{m_{i}}, \\
\dot{\mathbf{p}}_{i}=-\frac{\partial H}{\partial \mathbf{r}_{i}}=\mathbf{F}_{i},
\end{gathered}
$$


where the dot over a symbol means a time derivative, $m_{i}$ is the mass of the $i$-th particle, $H=\sum_{i=1}^{N}\left|\mathbf{p}_{i}\right|^{2} / 2 m_{i}+U_{\text {tot }}\left(\left\{\mathbf{r}_{i}\right\}\right)$ is the classical Hamiltonian of the system with $U_{t o t}$ being the total potential energy of the system, and $\mathbf{r}_{i}$ and $\mathbf{p}_{i}$ are three dimensional (3D) position and momentum of the $i$-th particle, respectively. Finally, $\mathbf{F}_{i}=-\partial U_{t o t} / \partial \mathbf{r}_{i}$ in Eq. $2.1 \mathrm{~b}$ is the total force on the $i$-th particle. The set of all particle coordinates and momenta $\left\{\mathbf{r}_{i}, \mathbf{p}_{i}\right\} \equiv \mathbf{r}, \mathbf{p}$ constitutes the total phase space of such Hamiltonian system. The divergence of the $6 N$-dimensional velocity field, $\left\{\dot{\mathbf{r}}_{i}, \dot{\mathbf{p}}_{i}\right\}$, generated by the Hamiltonian equations (Eqs. 2.1) at a time instance $t$ is called phase-space compressibility of our system (see e.g. [71])

$$
\kappa(\mathbf{r}, \mathbf{p})=\sum_{i=1}^{N}\left(\frac{\partial \dot{\mathbf{r}}_{i}}{\partial \mathbf{r}_{i}}+\frac{\partial \dot{\mathbf{p}}_{i}}{\partial \mathbf{p}_{i}}\right)
$$

It is a characteristic trait of all Hamiltonian systems that $\kappa(\mathbf{r}, \mathbf{p})=0$, as one can be easily convinced by direct calculation. This has the immediate consequence that the Lioville's theorem for a Hamiltonian system is satisfied, namely, that the phase-space volume element

$$
d \Gamma=\prod_{i=1}^{N} d \mathbf{r}_{i} d \mathbf{p}_{i}
$$

stays constant along the phase-space trajectories [71]. The stability of any numerical scheme for the integration of the equations of motion is closely related to the ability of such a scheme to fulfill the above requirement of the Lioville's theorem [71].

The most widely used (due to its numerical stability) discretized solution to the Hamiltonian equations is known as the velocity-Verlet integrator (see e.g. [71])

$$
\begin{aligned}
\mathbf{r}_{i}(t+\Delta t) & =\mathbf{r}_{i}(t)+\frac{\mathbf{p}_{i}(t)}{m_{i}} \Delta t+\frac{\mathbf{F}_{i}(t)}{2 m_{i}} \Delta t^{2} \\
\mathbf{p}_{i}(t+\Delta t) & =\mathbf{p}_{i}(t)+\frac{\mathbf{F}_{i}(t)+\mathbf{F}_{i}(t+\Delta t)}{2} \Delta t
\end{aligned}
$$

where the first equation is simply the second-order Taylor expansion of $\mathbf{r}_{i}(t+\Delta t)$, and the second equation is also obtained from the respective second-order Taylor expansion

$$
\mathbf{p}_{i}(t+\Delta t)=\mathbf{p}_{i}(t)+\mathbf{F}_{i}(t) \Delta t+\frac{\dot{\mathbf{F}}_{i}(t)}{2} \Delta t^{2}
$$


where $\dot{\mathbf{F}}_{i}(t)=\left(\mathbf{F}_{i}(t+\Delta t)-\mathbf{F}_{i}(t)\right) / \Delta t$ was used.

Alternatively, one can obtain the velocity-Verlet solution as a result of the direct action of the classical propagation operator, $\exp i \hat{L} t$, on a state in the phase space [71]

$$
\left(\begin{array}{l}
\mathbf{r}_{t} \\
\mathbf{p}_{t}
\end{array}\right)=e^{i \hat{L} t}\left(\begin{array}{l}
\mathbf{r}_{0} \\
\mathbf{p}_{0}
\end{array}\right),
$$

where the index 0 denotes an initial state of the system at $t_{0}=0$ and the operator $i \hat{L} t$ represents the classical Liouville operator defined as [71]

$$
i \hat{L}=\sum_{i=1}^{N}\left(\dot{\mathbf{r}}_{i} \frac{\partial}{\partial \mathbf{r}_{i}}+\dot{\mathbf{p}}_{i} \frac{\partial}{\partial \mathbf{p}_{i}}\right)
$$

Eq. 2.6 is just a formal consequence of the rewriting of the equation of motion (Eqs.2.1) through the Liouville operator as [71]

$$
\left(\begin{array}{c}
\dot{\mathbf{r}} \\
\dot{\mathbf{p}}
\end{array}\right)=i \hat{L}\left(\begin{array}{l}
\mathbf{r} \\
\mathbf{p}
\end{array}\right) \text {. }
$$

The form of the solution of the equations of motion (not only Hamiltonian ones) given by Eq. 2.6 is a very powerful tool, which opens the doors for the effective development and implementation of numerical integration schemes for systems with a Liouville operator of virtually any complexity [71, 72]. The key ingredient of any of such schemes is the factorization of complex propagators by applying the Trotter expansion (see e.g. [71, 72]). In our case the Liouville operator (Eq. 2.7) is straightforward to decompose into two contributions [71]

$$
i \hat{L}=i \hat{L}_{1}+i \hat{L}_{2}
$$

where the subpropagators $i \hat{L}_{1}$ and $i \hat{L}_{2}$ do not commute and are given by

$$
\begin{aligned}
& i \hat{L}_{1}=\sum_{i=1}^{N} \dot{\mathbf{r}}_{i} \frac{\partial}{\partial \mathbf{r}_{i}}=\sum_{i=1}^{N} \frac{\mathbf{p}_{i}}{m_{i}} \frac{\partial}{\partial \mathbf{r}_{i}} \equiv \frac{\mathbf{p}}{m} \frac{\partial}{\partial \mathbf{r}} \\
& i \hat{L}_{2}=\sum_{i=1}^{N} \dot{\mathbf{p}}_{i} \frac{\partial}{\partial \mathbf{p}_{i}}=\sum_{i=1}^{N} \mathbf{F}_{i} \frac{\partial}{\partial \mathbf{p}_{i}} \equiv \mathbf{F} \frac{\partial}{\partial \mathbf{p}}
\end{aligned}
$$

Now by applying the Trotter expansion we obtain up to the terms of the order of $\Delta t^{3}$ 


$$
e^{i \hat{L} \Delta t}=e^{i \hat{L}_{2} \Delta t / 2} e^{i \hat{L}_{1} \Delta t} e^{i \hat{L}_{2} \Delta t / 2}
$$

where $\Delta t$ is the time-step of the numerical integration procedure. Such symmetric factorization of the total propagator ensures that the resulting factorized operator retains the measure-preserving property discussed in the previous section [71]. Now following the lines of Ref. [72] we act subsequently with our factorized propagator (Eq. 2.11) on some initial state of the phase space at time $t_{0}=0$

$$
e^{i \hat{L}_{2} \Delta t / 2} e^{i \hat{L}_{1} \Delta t} e^{i \hat{L}_{2} \Delta t / 2}\left(\begin{array}{c}
\mathbf{r}_{0} \\
\mathbf{p}_{0}
\end{array}\right)=e^{i \hat{L}_{2} \Delta t / 2} e^{i \hat{L}_{1} \Delta t}\left(\begin{array}{c}
\mathbf{r}_{0} \\
\mathbf{p}_{\Delta t / 2}
\end{array}\right)
$$

where the action of the right most propagator on the initial state has left the coordinates intact and advanced the momenta from time 0 to $\Delta t / 2$ as

$$
\mathbf{p}_{\Delta t / 2}=\mathbf{p}_{0}+\mathbf{F}_{0} \frac{\Delta t}{2}
$$

Next, the action of $e^{i \hat{L}_{1} \Delta t}$ on the state $\left(\begin{array}{c}\mathbf{r}_{0} \\ \mathbf{p}_{\Delta t / 2}\end{array}\right)$ results in another intermediate state $\left(\begin{array}{c}\mathbf{r}_{\Delta t} \\ \mathbf{p}_{\Delta t / 2}\end{array}\right)$, where

$$
\mathbf{r}_{\Delta t}=\mathbf{r}_{0}+\Delta t \frac{\mathbf{p}_{\Delta t / 2}}{m}
$$

Finally, the last action of $e^{i \hat{L}_{2} \Delta t / 2}$ on $\left(\begin{array}{c}\mathbf{r}_{\Delta t} \\ \mathbf{p}_{\Delta t / 2}\end{array}\right)$ yields $\left(\begin{array}{c}\mathbf{r}_{\Delta t} \\ \mathbf{p}_{\Delta t}\end{array}\right)$ by taking the momenta from $t=\Delta t / 2$ to $t=\Delta t$ with the help of

$$
\mathbf{p}_{\Delta t}=\mathbf{p}_{\Delta t / 2}+\mathbf{F}_{\Delta t} \frac{\Delta t}{2}
$$

By inserting Eq. 2.13 into Eqs. 2.14 and 2.15 we recover the first and second velocityVerlet equations (Eqs. 2.4), respectively. In the above derivations the action of an exponential operator $e^{a \partial / \partial x}$ is determined by simply expanding it into Taylor series, which for $a \ll 1$ yields

$$
e^{a \frac{\partial}{\partial x}} x=\left(1+a \frac{\partial}{\partial x}\right) x=x+a
$$


Additionally, it should be noted that the action of $e^{a \partial / \partial x}$ on $f(x)$ returns the function $f(x+a)$ [71]. Here $x$ is any variable belonging to the phase space, i.e. either $\mathbf{r}$ or $\mathbf{p}$, and $f$ is the function defined over the whole phase space $\mathbf{r}, \mathbf{p}$ or over its subsets $\mathbf{r}$ and $\mathbf{p}$ separately. As an example of such function may serve the total potential energy of the system $U_{t o t}(\mathbf{r})$ or the force on a particular particle. Basically we see that the integration of the equations of motion is reduced to the sequential application of a set of translation operators, whose the most general form is given by Eq. 2.16, on the phase space of the system under investigation. This methodology was introduced by Martyna and his colleagues and is known as the direct translation technique [71, 73]. This technique makes it especially convenient to implement the numerical integration of the equations of motion in the computer code as a set of linear operations carrying out subsequent shifts of $\mathbf{r}$ and $\mathbf{p}$ in order to advance the system from time $t$ to $t+\Delta t$.

Giving Eqs. 2.4 one can generate temporal particle trajectories in the phase space and, assuming the studied system is ergodic, calculate various observables through the time averaging of the instant values along the trajectories. Ergodicity implies that averages in a respective statistical ensemble are equivalent to temporal averages in a molecular simulation [74].

Quite often it is possible to represent the interaction between atoms via pair interactions as a sum over all atom pairs in the simulation box. In order to mimic a behavior of an infinite system in a computer simulation one applies periodic boundary conditions (see e.g [70]), according to which the simulation box is periodically repeated in all dimensions. In this case for the calculation of the pair forces one only takes those periodic images of the particles that correspond to the shortest pair distances. This is called the minimal image convention [70]. Usually, to accelerate the force computation, one introduces a so-called cut-off radius, $r_{c}$, and considers for the force calculations only those pairs of atoms, which lie within $r_{c}$. The cut-off radius is chosen large enough such that $U\left(r_{c}\right)$ is close to zero in order to minimize the effect of the interaction truncation. Alternatively, for liquid systems the long-range corrections for the intermolecular distances larger than $r_{c}$ can be estimated analytically if the pair correlation function at these distances is approximately unity [70]. Further time savings are achieved with the help of the Verlet neighbor lists, according to which each particle has a list of its neighbors within $r_{c}+\Delta d$ [70], where $\Delta d$ is the so-called skin length. According to this procedure one does not have to loop over all particles in the simulation box to determine 
whether or not they lie within $r_{c}$, and the total force on a chosen particle is computed only from the pair contributions of neighboring particles that belong to the neighbor list. Typically the amount of particles and, thus, respective pair interactions, contained in the neighbor list (i.e. within a sphere of radius $r_{c}+\Delta d$ ) is much smaller compared to the total number of all atom pairs, $\sim N^{2}$, in the whole simulation box. In the course of a simulation the neighbor lists are typically updated when a mean-squared displacement of at least one particle in the simulation box exceeds the skin length, $\Delta d$. Sometimes it is more practical to use linked lists and a cell-structured simulation box instead of the Verlet neighbor list [70]. In this case to each cell of the simulation box a linked list is assigned that contains all the particles belonging to the respective cell.

\subsubsection{Velocity rescaling}

The sampling of the equations of motion according to Eqs. 2.4 naturally produces particle trajectories in a microcanonical ensemble $(N V E)$. In the majority of experiments though the temperature is held constant. The easiest way to fix the temperature would be to use a velocity rescaling.

The equipartition theorem (see e.g. Ref. [75]) allows to write the average kinetic energy of the system as

$$
\left\langle\sum_{i=1}^{N} \frac{m_{i} \mathbf{v}_{\mathbf{i}}^{2}}{2}\right\rangle=\frac{3}{2} N k_{b} T,
$$

where $\mathbf{v}_{\mathbf{i}}$ is the velocity of particle $i, k_{b}$ is the Boltzmann constant and $T$ is the current temperature of the system. Now let us assume that the velocities are rescaled every time-step, $\Delta t$, by a factor, $\lambda_{T}$, in order to yield a target temperature, $T_{0}$,

$$
\left\langle\sum_{i=1}^{N} \frac{m_{i}\left(\lambda_{T} \mathbf{v}_{\mathbf{i}}\right)^{2}}{2}\right\rangle=\frac{3}{2} N k_{b} \lambda_{T}^{2} T=\frac{3}{2} N k_{b} T_{0} .
$$

In this case it is straight forward to see that

$$
\lambda_{T}=\sqrt{\frac{T_{0}}{T}} .
$$

Such rescaling allows to fix the temperature at a desired value, but at the same time it completely eliminates temperature fluctuations. Therefore, the mean values calculated 
with the help of the velocity rescaling will correspond to the canonical ensemble (NVT) [76] but the distributions and dynamics will be, of course, wrong. In order to allow for temperature fluctuation the LAMMPS package for large-scale MD simulations gives an opportunity to perform the velocity rescaling in given time intervals, $d_{t}$, larger than the time-step [77]. This would correspond to the stochastic collisions of the simulated particles with imaginary thermostat particles at the average frequency of $1 / d_{t}$. Such frequency for a given system should be chosen in such a way that the particle positions and velocities fluctuate accordingly to the canonical ensemble [76]. Additionally, one can perform the rescaling of velocities only if the current temperature falls beyond some pre-set temperature window around $T_{0}$. The dynamics in such simulations will still not correspond to the true one for the NVT ensemble.

\subsubsection{Berendsen thermo- and barostats}

According to the Berendsen thermostat [78] the temperature is relaxed to the target one, $T_{0}$, such that the change of the temperature at every time-step is given by

$$
\frac{d T}{d t}=\frac{T_{0}-T}{\tau_{T}}
$$

where $\tau_{T}$ controls the rate of the temperature relaxation, which nominally corresponds to the velocity rescaling at every time-step by a factor of [78]

$$
\lambda_{T}=\left[1+\frac{\Delta t}{\tau_{T}}\left(\frac{T_{0}}{T}-1\right)\right]^{1 / 2}
$$

For the pressure relaxation to the target value, $P_{0}$, Berendsen has shown that the change in pressure for an isotropic system for a single time-step

$$
\frac{d P}{d t}=\frac{P_{0}-P}{\tau_{P}}
$$

technically corresponds to the simultaneous rescaling of the particle coordinates and system extents by the factor of [78]

$$
\lambda_{P}=\left[1-\frac{\Delta t \beta_{T}}{\tau_{P}}\left(P_{0}-P\right)\right]^{1 / 3},
$$


where $\tau_{P}$ steers the relaxation of pressure to the desired one, $P_{0}$, and $\beta_{T}=-(\partial V / \partial P) / V$ is the isothermal compressibility of the investigated system and $V$ is the volume of the system. The microscopic pressure, $P$, is calculated according to (see e.g. [70])

$$
P=\frac{N k_{b} T}{V}+\frac{\sum_{i=1}^{N} \mathbf{r}_{i} \mathbf{F}_{i}}{3 V}
$$

where the 1 st and 2 nd terms are the ideal gas and virial contributions, respectively. In both cases of thermo- and barostatting according to the Berendsen procedure the fluctuations of $T$ and $P$ are completely absent. Implying that despite the average values of the physical quantities of interest would correspond to the NVT and/or NPT ensembles, the fluctuations and dynamics will not. An extension of this method for the triclinic systems with simulation boxes of any shape is also possible [78].

\subsubsection{Extended-phase-space approach for simulations in canonical $(N V T)$ and constant stress $(\mathcal{P})$-temperature $(N \mathcal{P} T)$ ensembles}

We just saw that the usage of the Berendsen thermo- and barostats for the MD simulations in the $N V T$ or NPT ensembles literally kills the temporal fluctuations of $T$ and $P$. An extended-phase-space (or simply extended-variable) approach allows to carry out Molecular Dynamics simulations in the true $N V T, N P T$ and/or $N \mathcal{P} T$ ( $\mathcal{P}$ is the stress tensor) and preserves the true distribution functions and dynamics (see e.g. [79]). For this purpose equations of motion for additional unphysical variables, $\chi$, and their respective momenta, $\dot{\chi}$, as well as for the conventional physical variables, $\mathbf{r}$ and $\mathbf{p}$, are formulated and solved side by side. These unphysical degrees of freedom and the way they are coupled to the physical ones are chosen very carefully such that the distribution of the physical variables $\mathbf{r}$ and $\mathbf{p}$ corresponds to a desired statistical ensemble, after the unphysical variables have been integrated out [71-73, 80-82]. The total phase space of an extended system is then defined as a direct product of the phase space of the

physical degrees of freedom $\{\mathbf{r}, \mathbf{p}\}$ with the phase space of unphysical ones, $\{\chi, \dot{\chi}\}$. Extended systems are generally referred to as non-Hamiltonian ones. The reader can find a good introduction into the dynamics of the non-Hamiltonian systems in Refs. [71, 72]. Here I merely note that, as a rule, for the non-Hamiltonian systems $\kappa(\mathbf{r}, \mathbf{p}) \neq 0$ and the phase-space volume element $d \Gamma$ (Eq. 2.3) is no longer preserved. Instead, the invariant 
measure is a properly normalized volume element defined through the overall number of all degrees of freedom of the extended system as [71, 72]

$$
d \Gamma_{e x t}=\sqrt{\operatorname{det} G} d \chi d \dot{\chi} d \Gamma
$$

where $G$ is the metric tensor of the extended system, which is usually time-dependent. For a Hamiltonian system $\operatorname{det} G=1$.

The derivation of the computer simulation schemes for the non-Hamiltonian dynamics of extended systems is most conveniently done by using the formalism of the Liouville operator introduced in Sec. 2.1.1, which is readily extended for the case of extended-variable systems by defining the generalized Liouville operator as [71]

$$
i \hat{L}=\sum_{i=1}^{N}\left(\dot{\mathbf{r}}_{i} \frac{\partial}{\partial \mathbf{r}_{i}}+\dot{\mathbf{p}}_{i} \frac{\partial}{\partial \mathbf{p}_{i}}\right)+\dot{\chi} \frac{\partial}{\partial \chi}+\ddot{\chi} \frac{\partial}{\partial \dot{\chi}} .
$$

A generalized propagator of an extended system is defined through the generalized Liouville operator (Eq. 2.26) exactly as it has been previously done for the Hamiltonian system. The choice of a particular Trotter factorization scheme for the generalized propagator for the numerical integration of the equations of motion depends, of course, on a specific problem. In the case of NVT ensemble one typically couples a chain of additional thermostat variables to the momenta of physical particles. This is known as the Nosé-Hoover-chain method and was originally proposed by Martyna et al. [80]. For the isotropic barostatting (NPT ensemble) one couples the isotropic fluctuations of the volume to both real particles positions and momenta and then a Nosé-Hoover chain thermostat to both, real particles and volume fluctuations [81]. For a better temperature control a separate Nosé-Hoover chain thermostat can be assigned separately to the particles degrees of freedom and volume fluctuations. The equations of motion for the NVT and NPT ensembles are presented in Refs. [80] and [71, 81], respectively. The equations of motion for the case when the dimensions and shape of the simulation box at a fixed temperature may adjust to the very general conditions imposed by the external stress tensor, $\mathcal{P},(N \mathcal{P} T$ ensemble) are given by Shinoda et al. [82]. Generally speaking, no explicit discretization of the respective equations of motion is needed, because, as discussed above, the numerical integration can be realized by the means of the direct translation technique [71, 73]. For some relatively simple cases, such as simulations in 
$N V T$ and NPT ensembles, explicit integrators have been derived in the literature [71]. An explicit algorithm also exists for the case of the semi-isotropic NPT ensemble [81], i.e. when the diagonal components of the pressure tensor may be fixed independently and all off-diagonal elements are set to zero. The MD simulations in this thesis for the true $N V T$ and $N \mathcal{P} T$ ensembles (as described in this section) were performed in the LAMMPS package [77], which uses the very general form of the equations of motion given by Shinoda et al. [82] and follows the prescriptions for the numerical integration from Ref. [71].

\subsubsection{Multiple time-step scheme}

The multiple time-step MD method, the reversible reference system propagator algorithm, has been developed by Tuckerman and co-workers and is generally known under the alias rRESPA [71, 73, 83]. This methodology allows for the time reversible integration of the equations of motion for systems with molecular interactions that have various characteristic time scales. Below I am going to introduce the main idea behind the rRESPA techniques using a simple example following the lines of Ref. [71]. Multiple time-step algorithms may be most naturally obtained from the Liouville operator formalism described in Sec. 2.1.1. Assume for a moment that the Liouville operator has three contributions

$$
i \hat{L}=i \hat{L}_{1}+i \hat{L}_{2}+i \hat{L}_{3}
$$

where

$$
\begin{aligned}
i \hat{L}_{1} & =\frac{\mathbf{p}}{m} \frac{\partial}{\partial \mathbf{r}} \\
i \hat{L}_{2} & =\mathbf{F}_{\text {fast }} \frac{\partial}{\partial \mathbf{p}} \\
i \hat{L}_{3} & =\mathbf{F}_{\text {slow }} \frac{\partial}{\partial \mathbf{p}},
\end{aligned}
$$

where $\mathbf{F}_{\text {fast }}$ and $\mathbf{F}_{\text {slow }}$ are rapidly and slowly varying forces, respectively. The propagator for such Liouville operator is then written as [71]

$$
e^{i \hat{L} \Delta t}=e^{i \hat{L}_{1} \Delta t} e^{i \hat{L}_{2} \Delta t} e^{i \hat{L}_{3} \Delta t}=e^{i \hat{L}_{3} \Delta t / 2} e^{i \hat{L}_{1} \Delta t} e^{i \hat{L}_{2} \Delta t} e^{i \hat{L}_{3} \Delta t / 2}
$$


where in the last step the Trotter expansion (see Eq. 2.11) was used. Now by applying again the Trotter expansion for the product of subpropagators $e^{i \hat{L}_{1} \Delta t} e^{i \hat{L}_{2} \Delta t}$ we arrive at the final expression for the factorized propagator for the case of two time-steps, $\Delta t$ and $\Delta t / n,[71]$

$$
e^{i \hat{L} \Delta t}=e^{i \hat{L}_{3} \Delta t / 2}\left[e^{i \hat{L}_{2} \Delta t / 2 n} e^{i \hat{L}_{1} \Delta t / n} e^{i \hat{L}_{2} \Delta t / 2 n}\right]^{n} e^{i \hat{L}_{3} \Delta t / 2} .
$$

The operator in the square brackets advances the system in time with the time-step $\Delta t / n$ $\mathrm{n}$ times accordingly to the fast varying force $\mathbf{F}_{\text {fast }}$, whereas the system is propagated only twice per time-step $\Delta t$ for the slow motions according to $\mathbf{F}_{\text {slow }}$. Such approach appears to be indispensable for reaching larger simulation times, and, thus, better statistics, in the simulations of basically all molecular systems, because typically chemical bonds in molecules fluctuate much faster compared to the slow characteristic motion due to Van der Waals intermolecular forces.

\subsection{Monte Carlo simulation}

In contrast to the Molecular Dynamics discussed above, one does not normally simulate a propagation of a system in a physical space-time in a typical Monte Carlo (MC) simulation, but rather generates a stochastic sequence of molecular configurations according to the chosen statistical ensemble and computes the observables as ensemble averages over these configurations. A very nice and comprehensible account of Monte Carlo simulations is given by Landau and Binder in Ref. [84] as well as in the book of Frenkel and Smit [74]. It should be also mentioned that an extension of the traditional Monte Carlo approach, a so-called kinetic Monte Carlo [85], also exists that enables the simulation of temporal evolution. This method allows to simulate physical times that in principle may be much larger compared to the ones from the MD method (depending on the application), but strictly speaking such temporal evolution is not deterministic and, thus, lacks of the time reversibility. In this section we limit ourselves with the classical Monte Carlo method.

For a classical system the probability of the $n$-th microscopic state with some particular choice of $\left\{\mathbf{r}_{i}, \mathbf{p}_{i}\right\}$ is given by [74]

$$
P_{n}=\frac{e^{-\beta\left(\sum_{i=1}^{N} \mathbf{p}_{i}^{2} /(2 m)+U\left(\left\{\mathbf{r}_{i}\right\}\right)\right)}}{h^{3 N} N ! Q},
$$


where $h$ is the Planck constant, $Q$ is the partition function

$$
\begin{aligned}
Q & =\frac{\int \prod_{i=1}^{N} d \mathbf{r}_{i} d \mathbf{p}_{i} e^{-\beta\left(\sum_{i=1}^{N} \mathbf{p}_{i}^{2} /(2 m)+U\left(\left\{\mathbf{r}_{i}\right\}\right)\right)}}{h^{3 N} N !} \\
& =\left(\frac{2 m \pi^{1 / 2}}{\beta}\right)^{3 N} \frac{\int \prod_{i=1}^{N} d \mathbf{r}_{i} e^{-\beta\left(U\left(\mathbf{r}_{i}\right)\right)}}{h^{3 N} N !} \\
& =\left(\frac{2 m \pi^{1 / 2}}{\beta}\right)^{3 N} Z,
\end{aligned}
$$

where $Z=\int \prod_{i=1}^{N} d \mathbf{r}_{i} e^{-\beta U\left(\mathbf{r}_{i}\right)} /\left(h^{3 N} N\right.$ !). Giving the above expression the probability of the $n$-th microscopic state $\left\{\mathbf{r}_{i}, \mathbf{p}_{i}\right\}$ takes on the following form

$$
P_{n}=\frac{e^{-\beta U_{n}}}{Z}
$$

where $U_{n} \equiv U\left(\left\{\mathbf{r}_{i}\right\}\right)$ is the potential energy corresponding to the $n$-th microscopic state. To generate a stochastic sequence of states giving the above probability is basically unrealistic, because $Z$ is quite often unknown or computationally very difficult to be calculated (one has to calculate a $3 \mathrm{~N}$-dimensional configurational integral for that). So, instead, one generates the sequence of states of the system as a Markov chain using the transition probability $W_{n-1 \mid n}$ for the transition from the state $n-1$ to the state $n$. A defining property of the Markov chain is that $W_{n-1 \mid n}$ depends only on the preceding state. An encyclopedic overview of the Markov chains is given in the handbook of stochastic methods by Gardiner [86]. In the equilibrium the exact choice of $W_{n-1 \mid n}$ is dictated by the detailed balance condition [74]

$$
P_{n-1} W_{n-1 \mid n}=P_{n} W_{n \mid n-1},
$$

meaning that the conditional probability of being in the state $n$ if the preceding state was $n-1$ equals to the conditional probability of being in the state $n-1$ if the preceding state was $n$. It follows from Eq. 2.34

$$
W_{n-1 \mid n}=\frac{P_{n}}{P_{n-1}} W_{n \mid n-1}=e^{-\beta\left(U_{n}-U_{n-1}\right)} W_{n \mid n-1} .
$$


By choosing

$$
W_{n-1 \mid n}=\min \left(1, e^{-\beta\left(U_{n}-U_{n-1}\right)}\right)
$$

the detailed balance condition (Eq. 2.34 will be fulfilled. The above choice of $W_{n-1 \mid n}$ also means that the state of lower energy is always accepted. Such sampling scheme is called the Metropolis Monte Carlo method and was introduced for the first time by Metropolis et al. for the computation of the equation of state of the 2D hard spheres [87]. The usage of the cut-off radius, periodic boundary conditions and neighbor lists is readily incorporated into the Monte Carlo scheme [70, 84, 88]. For the Monte Carlo simulations performed in this thesis (see Chap. 3) I have created my own C++ objectorient source code.

\subsection{Liquid-state theory}

In the contemporary times numerical simulation techniques, such as Molecular Dynamics and/or Monte Carlo methods discussed above, are the main tools to study the molecular-scale structure and properties of materials. Nevertheless, it is always nice to have a simple theory, which can help to interpret the results of a molecular simulation. In this section I describe the classical liquid-state theory that is later employed to rationalize the equation of state of various models for the atomistic simulation of liquid mercury. This approach has been also presented in one of my recent publication [89]. The liquid-state theory is based on the routinely used Weeks-Chandler-Andersen (WCA) decomposition of the pair potential $U(r)$ into the reference repulsive part $U_{r}(r)$ and purely attractive interaction $U_{a}(r)$ as [90, 91]

$$
U(r)=U_{r}(r)+U_{a}(r),
$$

where

$$
U_{r}(r)= \begin{cases}U(r)-U\left(r_{\text {min }}\right) & \text { if } r \leq r_{\text {min }} \\ 0 & \text { otherwise }\end{cases}
$$

and

$$
U_{a}(r)= \begin{cases}U\left(r_{\text {min }}\right) & \text { if } r \leq r_{\text {min }} \\ U(r) & \text { otherwise }\end{cases}
$$


with $r_{\min }$ being the minimum of the pair potential $U(r)$, and $r$ is the distance between two atoms. The free energy of the system will consist then out of the ideal gas contribution, $F_{i d}$, and two contributions $F_{r}$ and $F_{a}$ due to the repulsive and attractive parts of the pair potential, respectively,

$$
\frac{\beta}{V} F=F_{i d}+F_{r}+F_{a},
$$

where $\beta=1 /\left(k_{b} T\right)$, and the prefactor in front of $F$ is chosen out of convenience. The ideal gas contribution is given by (see e.g. Ref. [75])

$$
F_{i d}=\rho \ln \frac{\rho \Lambda_{\mathrm{T}}^{3}}{e}
$$

where $\Lambda_{\mathrm{T}}=\sqrt{\frac{2 \pi \hbar^{2} \beta}{m}}$ is the de-Broglie wavelength, $m$ is the mass of an atom, and $\hbar$ is the reduced Plank constant, and $\rho$ is the number density. The free energy of the reference repulsive system is obtained from the Carnahan-Starling equation of state [92] and reads as [93]

$$
F_{r}=\rho \frac{4 \eta-3 \eta^{2}}{(1-\eta)^{2}}
$$

with $\eta$ being the packing fraction defined as

$$
\eta=\frac{\pi \rho d^{3}}{6}
$$

where $d$ is the effective hard-sphere (HS) diameter of an atom and is given by [94]

$$
d=\int_{0}^{r_{\min }} d r\left(1-e^{-\beta U_{r}(r)}\right) .
$$

Finally, the free energy of the purely attractive part of the pair potential is obtained from the Barker-Henderson perturbation theory [94], according to which the attraction is treated as the perturbation to the reference repulsive system. The first non-vanishing contribution due to the attraction to the free energy is given by [91, 94]

$$
F_{a}=\frac{\rho^{2}}{2} \int_{0}^{r_{\mathrm{c}}} d^{3} r \beta U_{a}(r) g_{h s}(r),
$$


where $g_{h s}(r)$ is the HS pair correlation function [95].

Giving Eq. 2.40 one can now estimate the free energy for a desired thermodynamic state characterized by a given pair of $\rho$ and $T$ values. In order to calculate the coexistence density the free energy versus density curve, $F(\rho)$, is constructed and a doubletangent construction [96] is used as illustrated in the following chapter. 


\section{Simulation of the surface of liquid mercury}

Mercury is the only known metallic element, which is liquid at room temperatures and has a very high density of $\sim 13550 \mathrm{~kg} / \mathrm{m}^{3}$ [97]. This is approximately 13.5 times larger than that of water. Moreover, unlike conventional liquids, the surface of liquid mercury at room temperature features a strong surface layering at the boundary to a less denser substance [98] and an extremely high surface tension, i.e. $\gamma \approx 0.487 \mathrm{~N} / \mathrm{m}$ for the mercury/air interface [99]. For comparison the surface tension of water at the interface with air only amounts to $\sim 0.07 \mathrm{~N} / \mathrm{m}$ [99]. Chemically mercury is a very inert element, meaning that it is hard to oxidize [63]. Additionally, at the temperature of liquid helium mercury becomes superconducting [100]. The metallic nature of mercury appears only in the clusters of more than 100 atoms [101]. Most of the peculiar properties of liquid mercury are attributed to relativistic effects [63, 101, 102], according to which the $6 \mathrm{~s}$ electrons move at velocities comparable to the speed of light under the influence of the high positive charge of the $\mathrm{Hg}$ ion core, and, respectively, their masses are magnified leading to the shrinkage of the $6 s$ electron orbital compared to the other elements of the same group (e.g. zinc or cadmium) [63].

Due to its unique properties mercury is widely used in organic electronics in the form of a drop-electrode, which can be easily manipulated at room temperature (see e. g. Ref. [50]). Moreover, liquid mercury can be used to produce highly conducting nanowires [103-106]. On the other hand, mercury is used as a main constituent or produced as a byproduct in energy production [107], fluorescent bulbs [108], gold mining [109] etc. Because of the high toxicity of mercury [109-111] the reduction of the mercury emission into the environment is one of the main issues related to the usage of 
mercury. For this purpose various organic systems were designed for the localization of mercury [112-114]. Intriguing structures have been recently discovered by irradiating alkylthiol monolayers on the surface of liquid mercury with high frequency sonic waves [115]. These experiments revealed the formation of sheet-like monocrystallites consisting of a mercury backbone with thiol molecules symmetrically attached to it from two sides. Evidence for a possible existence of such structures has been also seen before [62]. The exact mechanism of formation and structure of such aggregates is not yet completely understood.

A thermodynamically robust framework for the large-scale simulation of mercury allowing us to predict its behavior for these applications would facilitate further progress in these areas. Substantial efforts over the last decades have been directed into this direction [101, 116-129]. In this chapter I investigate the surface tension and density profiles of the surface of liquid mercury at the room temperature, $T=293 \mathrm{~K}$, by means of classical MD techniques. With this perspective atomistic models such as the DI double-exponent potential [121, 130] and its empirical density-dependent (DD) modification [126], as well as the Raabe potential [120] and recently proposed embedded-atom models (EAMs) for liquid $\mathrm{Hg}$ [131-133] are evaluated in detail. The results, which I obtained in the current chapter, have been recently published in my joint paper together with D. Bedrov and M. Müller [89], and, the outline and discussions of this chapter follow closely this paper. First, I cover various models of liquid mercury for atomistic simulations available in the literature. Afterwards I present the respective results for the surface tension and density profiles. As Louis has pointed out, an ad-hoc inclusion of a density-dependent coefficients into a density-independent pair potential may cause undesirable and at first sight non-obvious artifacts [134]. This is why I investigate with special care the effect of the density dependence of the mercury interactions on the liquid-vapor coexistence of liquid mercury. The simulation results on the phase behavior of liquid mercury are in good agreement with the semi-analytic calculations from the classical liquid-state theory. Moreover, complementary Monte Carlo simulations support my conclusions for the DI and DD models. As the reader shall see, the EAM models yield values of the surface tension that are the closest to the experimental one. On the contrary, the DI model results in the lowest value of the surface tension, but at the same time it is the most computationally efficient and thermodynamically robust model among the studied ones in this work. Thus, the question is posed if it is possible 
to choose a parameter set for the DI model that would improve the agreement of the surface tension with experiment while preserving the experimental coexistence density of the liquid mercury with its vapor. In the end of this chapter I answer this question positively and present a corresponding optimization procedure, which can be applied at any desired temperature.

\subsection{Models of liquid mercury in the literature and the need for a new model}

\subsubsection{Lennard-Jones model}

The simplest model for liquid mercury is a Lennard-Jones liquid, in which each interaction center represents a single $\mathrm{Hg}$ atom. In the Lennard-Jones (LJ) potential

$$
U(r)=4 \varepsilon\left[\left(\frac{\sigma_{0}}{r}\right)^{12}-\left(\frac{\sigma_{0}}{r}\right)^{6}\right]
$$

$\sigma_{0}$ and $\varepsilon$ denote characteristic length and energy scales, which one has to determine in order to relate the model to experimental values of the coexistence density and surface tension of liquid mercury. Since the surface tension of liquid mercury at the room temperature is extremely large, this thermodynamic state is close to the triple point of the LJ fluid. At this point the vapor, liquid and crystal phases coexist with each other and the liquid-vapor surface tension, $\gamma$, is the highest. In the LJ model the triple point occurs at the values of the reduced temperature, $T^{*}$, density, $\rho^{*}$, and liquid-vapor surface tension, $\gamma^{*}$, of $0.7,0.8$ [135, 136], and 1.137 [137], respectively. These reduced dimensionless quantities are connected with the conventional ones by [135-137]

$$
\begin{aligned}
T^{*} & =k_{\mathrm{B}} T / \varepsilon \\
\rho^{*} & =\rho \sigma_{0}^{3} \\
\gamma^{*} & =\gamma \sigma_{0}^{2} / \varepsilon
\end{aligned}
$$

Let us assume that the $\mathrm{LJ}$ system has the same triple point as the liquid $\mathrm{Hg}$ at $T=235 \mathrm{~K}$ and $\rho=13.69 \mathrm{~g} / \mathrm{cm}^{3}$ in order to recover $\varepsilon$ and $\sigma_{0}$. This yields $\varepsilon \approx 0.66713 \mathrm{kcal} / \mathrm{mol}$, 
$\sigma_{0} \approx 2.695896 \AA$ and, consequently, the LJ prediction for the maximal value of liquidvapor surface tension of $\sim 0.07 \mathrm{~N} / \mathrm{m}$. We see that such value is considerably smaller than the experimental one, $\sim 0.5 \mathrm{~N} / \mathrm{m}$, at the room temperature. This estimation of the surface tension of liquid mercury from the LJ model unambiguously discloses the inadequacy of the LJ description of the surface of the liquid mercury and demonstrates a clear need for a more advanced model, which can simultaneously reproduce the liquid density and surface tension of liquid mercury at room temperatures.

\subsubsection{Density-independent double-exponent model}

Early pair potentials for the interactions in the bulk of liquid mercury derived from the experimental structure factors demonstrated that the pair interaction of liquid mercury was strongly dependent on the thermodynamic state (defined by temperature and density) of mercury bulk systems [117, 118]. In order to take this into account in a more systematic way a previously proposed double-exponent potential for liquid metals [130] was made state-dependent by adjusting the interaction strength, $A_{1}$, in a wide temperature rage in order to mimic the liquid part of the mercury phase diagram [121]. The resulting potential is given by [121]

$$
U(r)=A_{0} e^{-a r}-A_{1}(T) e^{-b\left(r-R_{0}\right)^{2}},
$$

where the values of the parameters at $T=293 \mathrm{~K}$ are $A_{0}=1901990.928 \times 10^{9} \mathrm{kcal} / \mathrm{mol}$, $A_{1}=0.971 \mathrm{kcal} / \mathrm{mol} a=12.48 \AA^{-1}, b=0.44 \AA^{-2}$ and $R_{0}=3.56 \AA$ [121]. The first term describes the Born-Meyer repulsion and the second term in Eq. 3.3 determines the attractive part of the potential, which has the minimum at $R_{0}$. The parameter $b$ is the inverse squared range of the attractive interaction. Despite being successful in describing Hg bulk systems, this model failed to reproduce a strongly stratified surface of liquid mercury [122].

\subsubsection{Density-dependent double-exponent model}

A decent agreement with the experimental density profiles has been obtained in the self-consistent quantum Monte Carlo [123, 124] and in the ab initio Molecular Dynamics [125] simulations. In each of these approaches the computation of the interatomic 
interactions involves regular recalculations of the respective electronic densities via the Kohn-Sham equation [138] to guarantee that effective density-dependent potentials used to move mercury ions correspond to their actual configuration. Such computer simulation schemes will, though, impose a tremendous computational penalty on the intended large-scale simulations of SAM on liquid $\mathrm{Hg}$ limiting them in time and number of particles. As an alternative the DI model (Eq. 3.3) was ad-hoc modified in attempt to capture the metal-nonmetal transition (as the local density of liquid $\mathrm{Hg}$ falls approaching the surface) by making the coefficient $A_{1}$ density-dependent (DD) [126]

$$
A_{1}=f_{i} f_{j} A_{1}^{M M}+\left[f_{i}\left(1-f_{j}\right)+\left(1-f_{i}\right) f_{j}\right] A_{1}^{M V}+\left(1-f_{i}\right)\left(1-f_{j}\right) A_{1}^{V V},
$$

where $A_{1}^{M M}=0.971 \mathrm{kcal} / \mathrm{mol}, A_{1}^{M V}=1.942 \mathrm{kcal} / \mathrm{mol}, f_{i} \equiv f\left(\rho\left(\mathbf{r}_{\mathbf{i}}\right)\right)$ is a function of the local density $\rho\left(\mathbf{r}_{i}\right)$ at the position of $i$-th atom, and is given by $f(\rho)=1$ if the density is larger than a threshold value $11000 \mathrm{~kg} / \mathrm{m}^{3}$ and it vanishes for $\rho<8000 \mathrm{~kg} / \mathrm{m}^{3}$, and is chosen to be a smooth function in between. Thus only if the local density at the position of a particle is in the intermediate density interval between 8000 and $11000 \mathrm{~kg} / \mathrm{m}^{3}$ does the density dependence of the potential matter. I have tested polynomials of $1 \mathrm{st}, 2 \mathrm{nd}$, 3 rd and 5th order to represent $f(\rho)$, and found that the functional form of $f(\rho)$ does not influence the phase behavior. In my MD simulations I use the $3 \mathrm{rd}$ order polynomial for $f(\rho)$ in order to ensure the continuity of the forces. In the approach from Ref. [126] the form of the density profiles was biased by a pre-determined reference density profile, which is not quite physical and limits the simulations to a few simple geometries (planar and spherical). Therefore, for the calculation of the local density, $\rho\left(\mathbf{r}_{\mathbf{i}}\right)$, at the position of $i$-th particle, $\mathbf{r}_{\mathbf{i}}$, I adopted a standard weighting function method [139], which ensures that the calculation of the local density is independent of geometry. According to this method the local density may be calculated as

$$
\rho\left(\mathbf{r}_{\mathbf{i}}\right)=\sum_{j \neq i} w\left(r_{i j}\right)
$$

with

$$
w\left(r_{i j}\right)=A_{n o r m}\left(r_{i j}^{2}-r_{c}^{2}\right)^{2},
$$


and $r_{i j}=\sqrt{\left(\mathbf{r}_{\mathbf{i}}-\mathbf{r}_{\mathbf{j}}\right)^{2}}$ being the distance between atoms $i$ and $j$. The normalization factor $A_{\text {norm }}$ I define by the condition

$$
4 \pi \int_{0}^{r_{\mathrm{c}}} d r r^{2} w(r)=1
$$

where $r \equiv r_{i j}$ and $r_{\mathrm{c}}=9 \AA$ is the cut-off radius of the pair potential. Having a geometryindependent force field for liquid $\mathrm{Hg}$ is very important for the simulation of the systems, which are relevant for applications. For example, this is important for the systems with a varying shape of $\mathrm{Hg}$ droplets (see e.g. Ref [51]). Ref. [126] gives no estimate for the coefficient $A_{1}^{V V}$, and, instead, it is said therein that its value does not influence the result much. Therefore, in order to obtain consistent results in the limit of low and high densities, I have chosen $A_{1}^{V V}$ to be equal to $A_{1}^{M M}$.

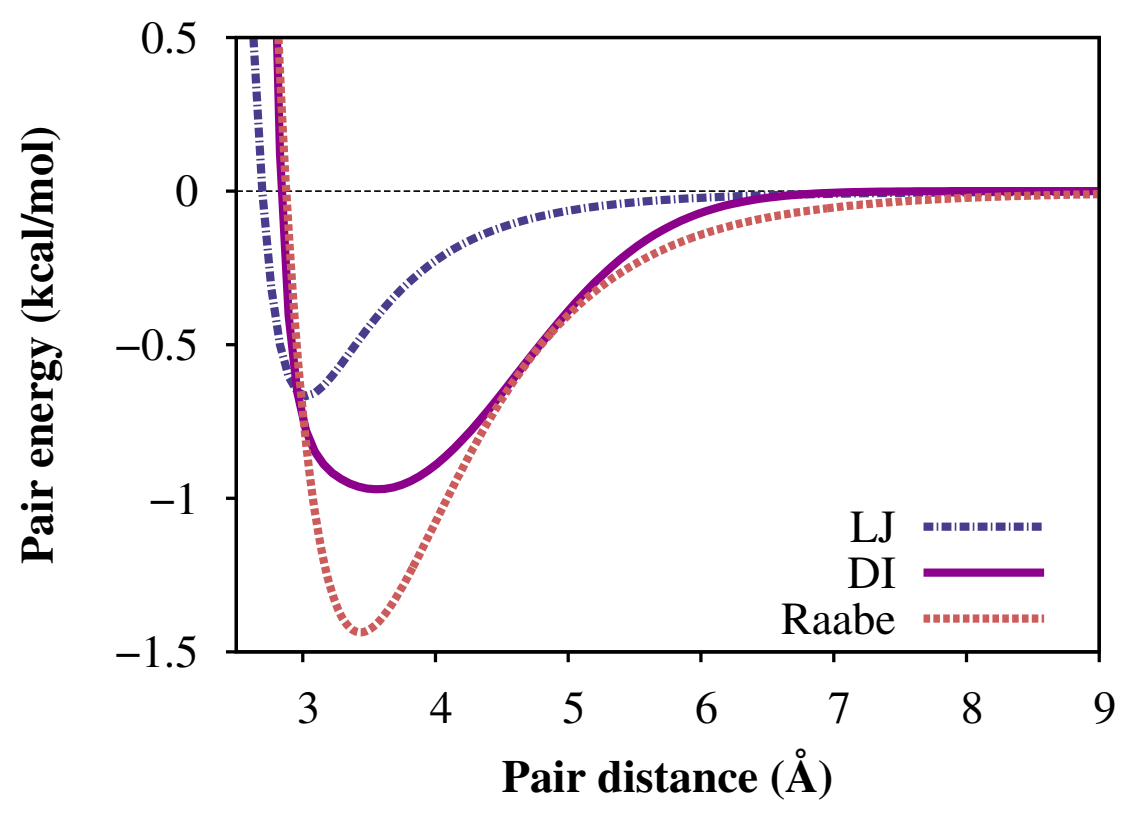

Figure 3.1: Comparison of the LJ, DI and Raabe pair potentials for liquid mercury. The $\mathrm{LJ}$ potential is plotted for the parameters derived in Sec. 3.1.1 at $T=235 \mathrm{~K}$. The DI and Raabe potentials are plotted for $T=293 \mathrm{~K}$. 


\subsubsection{Raabe model}

The Raabe model is another pair potential model evaluated in the following. It is given by [120]

$$
U(r)=\lambda_{E} \sum_{j=3}^{9} a_{2 j}\left(\lambda_{r} r\right)^{-2 j}-a_{s o} r^{-\alpha_{s o}} \exp ^{-\beta_{s o} r}+\frac{C_{9}(T)}{r^{9}} .
$$

The first two terms in the above equation represent the Schwerdtfeger et al. potential, which takes into account relativistic and spin-orbit effects [101]. The set of parameters from Ref. [101] designated therein as "cc-ULB+SO" is used for the first term. Parameters for the second term are given in Ref. [101] as well. The last term in Eq. 3.8 is an empirical temperature-dependent long-range correction proposed by Raabe et al., which allows to reproduce the experimental phase diagram of mercury for a wide range of temperatures starting from $\sim 400 \mathrm{~K}$ [120]. The properties of this model for the surface of liquid $\mathrm{Hg}$ at room temperatures have not been reported in the previous works. Fig. 3.1 offers a comparison of the density independent pair potential for liquid mercury discussed so far. Namely the above figure compares the LJ (Eq. 3.1), DI (Eq. 3.3) and Raabe pair potentials with each other. The reader can see that the LJ potential appears to have a much shorter range of the attractive interaction compared to the DI and Raabe models. On the contrary the Raabe model has the largest attractive range and much stronger "attraction strength" compared to the LJ and DI models. Because of the extended range of attraction of the Raabe model I used the cut-off radius, $r_{c}$, of $11 \AA$ for this model. As is clear from Fig. 3.1, the double-exponent DI potential has a substantially extended attraction well, which, as will become apparent later, is crucial for the correct phase behavior of mercury at low temperatures $(T<400 \mathrm{~K})$. According to my simulations the Raabe model results in the crystallized bulk of mercury already at $T=293 \mathrm{~K}$. Obviously, the too strong attraction strength and long-ranged interaction (compared to the DI model) promote the Hg crystallization in this model. Giving this, I discard the Raabe model in the following discussion.

\subsubsection{Embedded-atom models}

The embedded-atom method (EAM) was initially developed in the beginning of 1980's by Daw and Baskes for the investigation of the hydrogen behavior in and its influence 
on the ground-state properties of Nickel (Ni) and Palladium (Pd) [140, 141]. Later on this method was applied to the transition metals by Foiles and colleagues [142144]. Only very recently, in 2006, this method was extended and used for the atomistic simulation of the bulk of liquid mercury by Belashchenko [133]. In 2013 Belashchenko proposed an embedded-atom model for the case of a strongly compressed bulk of liquid mercury [131, 132]. Below I am going to present both of Belashchenko's EAM models, which I designate in the following as EAM2006 and EAM2013 models, respectively. Generally, a potential energy per atom $i$ at the position $\mathbf{r}_{\mathbf{i}}$ consists of two contributions, an embedded density-dependent energy and a sum of pairwise interactions of the $i$-th atom with its neighbors, and it has the following form (see e.g. Ref. [133])

$$
U_{i}=U_{e m}\left(\rho_{d l}\left(\mathbf{r}_{\mathbf{i}}\right)\right)+\sum_{j \neq i} U\left(r_{i j}\right)
$$

where $U_{e m}$ is the embedding energy due to the many-body electronic interactions, $\rho_{d l}$ is an effective (dimensionless) electron density at position $\mathbf{r}_{i}$, and $U(r)$ is the pair interaction between the $\mathrm{Hg}$ ions. The form of Eq. 3.9 is dictated by the density-functional theory [141]. Thus, the EAM methodology represents a generic framework for the treatment of many-body effects due to underlying electron structure of metals in atomistic simulations. For the EAM2013 model $U_{e m}$ is given by [131, 132]

$$
\begin{aligned}
& U_{e m}=a_{1}+c_{1}\left(\rho_{d l}-\rho_{0}\right)^{2}, \text { with } \rho_{1}<\rho_{d l} \leq \rho_{8}, \\
& U_{e m}=a_{i}+b_{i}\left(\rho_{d l}-\rho_{i-1}\right)+c_{i}\left(\rho_{d l}-\rho_{i-1}\right)^{2}, \text { with } \rho_{i}<\rho_{d l} \leq \rho_{i-1}, i=2, \ldots, 7, \\
& U_{e m}=\left[a_{8}+b_{8}\left(\rho_{d l}-\rho_{7}\right)+c_{8}\left(\rho_{d l}-\rho_{7}\right)^{2}\right]\left[2 \frac{\rho_{d l}}{\rho_{7}}-\left(\frac{\rho_{d l}}{\rho_{7}}\right)^{2}\right], \text { with } \rho_{d l} \leq \rho_{7}, \\
& U_{e m}=a_{9}+b_{9}\left(\rho_{d l}-\rho_{8}\right)+c_{9}\left(\rho_{d l}-\rho_{8}\right)^{m}, \text { with } \rho_{8}<\rho_{d l} \leq \rho_{9}, \\
& U_{e m}=a_{10}+b_{10}\left(\rho_{d l}-\rho_{9}\right)+c_{10}\left(\rho_{d l}-\rho_{9}\right)^{n}, \text { with } \rho_{d l}>\rho_{9},
\end{aligned}
$$

where $a_{1}=-2.0292147, a_{2}=-1.8096637345, a_{3}=-1.69697, a_{4}=-1.3531480$, $a_{5}=-1.188998, a_{6}=-0.884708, a_{7}=-0.132113456, a_{8}=-1.2932, a_{9}=-1.3034$, $a_{10}=60.5636636 \mathrm{kcal} / \mathrm{mol} ; b_{2}=-3.991865, b_{3}=1.1745827, b_{4}=-8.051217, b_{5}=$ $4.4036127, b_{6}=-13.09772987, b_{7}=-5.71708987, b_{8}=19.3770861, b_{9}=7.2579369$, 
$b_{10}=60.65324613 \mathrm{kcal} / \mathrm{mol} ; c_{1}=18.144842, c_{2}=-32.2903, c_{3}=46.129, c_{4}=$ $-69.1935, c_{5}=125.00959, c_{6}=-46.129, c_{7}=-73.8064, c_{8}=92.258, c_{9}=22.6032$, $c_{10}=5.304835 \mathrm{kcal} / \mathrm{mol} ; \mathrm{m}=1.7, \mathrm{n}=3$, as well as $\rho_{0}=1, \rho_{1}=0.89, \rho_{2}=0.81$, $\rho_{3}=0.71, \rho_{4}=0.62, \rho_{5}=0.55, \rho_{6}=0.47, \rho_{7}=0.3, \rho_{8}=1.2, \rho_{9}=2.8$; and $1 \mathrm{eV}=$ $23.0645 \mathrm{kcal} / \mathrm{mol}$ was used to transfer original units [131] to $\mathrm{kcal} / \mathrm{mol}$.

And for the EAM2006 model $U_{e m}$ takes the next form [133]

$$
\begin{aligned}
& U_{e m}=a_{1}+a_{2}\left(\rho_{d l}-\rho_{0}\right)^{2}+a_{3}\left(\rho_{d l}-\rho_{0}\right)^{3}, \text { with } \rho_{d l} \geq 0.8 \rho_{0}, \\
& U_{e m}=a_{4} \sqrt{\rho_{d l}}+a_{5} \rho_{d l}, \text { with } \rho_{d l}<0.8 \rho_{0},
\end{aligned}
$$

where $a_{1}=-3.7549, a_{2}=1.415, a_{3}=31.4369, a_{4}=-2.5710459$ and $a_{5}=-1.31755956$ $\mathrm{kcal} / \mathrm{mol}$, as well as $\rho_{0}=1[133]$. The effective density $\rho_{d l}$ is determined by

$$
\rho_{d l}\left(\mathbf{r}_{\mathbf{i}}\right)=\sum_{j \neq i} \tilde{\psi}\left(r_{i j}\right)
$$

with

$$
\tilde{\psi}(r)=p_{1} \exp \left[-p_{2} r\right]
$$

where for EAM2013 and EAM2006 $p_{1}=4.8019$ [131] and 4.7986 [133], respectively; in both cases $p_{2}=1.3095 \AA$ [131, 133]. The effective pair potential $U(r)$ at $T=293 \mathrm{~K}$ for both models for $2.55 \leq r \leq 8.35 \AA$ is tabulated in Ref. [133], and for $0 \leq r<2.55 \AA$ is defined as [131]

$$
U(r)=(3.906111462-146.32856864(2.55-r)+87.645[\exp (1.96(2.55-r))-1])
$$

where the units of energy are $\mathrm{kcal} / \mathrm{mol}$. At the cut-off radius $r_{c}=8.35 \AA$ the pair potential is set to zero. The minimum of $U(r)$ is $-1.423 \mathrm{kcal} / \mathrm{mol}$ at $r_{\min }=3.18 \AA$.

Finally, in order to calculate the free energy of the EAM models according to the liquid-state theory discussed in Sec. 2.3 the contribution due to the embedded energy, $U_{e m}\left(\rho_{d l}\right)$, has to be added to the free energy represented by Eq. 2.40 . This contribution is given simply by

$$
F_{e m}=\beta \rho U_{e m}\left(\left\langle\rho_{d l}\right\rangle\right),
$$

where the bulk average effective dimensionless density $\left\langle\rho_{d l}\right\rangle$ is calculated from the num- 
ber density of $\mathrm{Hg}$ ions, $\rho$, as [133]

$$
\left\langle\rho_{d l}\right\rangle=4 \pi \rho \int_{0}^{\infty} g(r) \tilde{\psi}(r) r^{2} d r
$$

In my calculations I approximate $g(r)$ with the one for the hard spheres, which appears to be a very good approximation in this case, because the so-computed value of $\left\langle\rho_{d l}\right\rangle$ deviates only by $0.7 \%$ from the corresponding value obtained by using the experimental $g(r)[145]$ for $T=293 \mathrm{~K}$.

\subsubsection{Simulation details}

\section{Molecular Dynamics}

The LAMMPS package [77] is used to carry out MD simulations of the mercury film. It was extended by three $\mathrm{C}++$ modules in order to implement the double-exponent DI and DD potentials, as well as the Raabe potential. The film of liquid mercury was prepared by first initializing $N=2 \times 10^{3} \mathrm{Hg}$ atoms on a simple cubic lattice with $L_{\mathrm{x}}=L_{\mathrm{y}}=24.56 \AA$ and $L_{\mathrm{z}}=80.673 \AA$. Such bulk system was equilibrated for $8 \mathrm{~ns}$. Second, the empty space above and below the $\mathrm{Hg}$ film along the z-axis was added, so that the film would not feel the presence of walls if they were placed at both ends of the system along the z-axis. Afterwards the film is equilibrated for another $2 \mathrm{~ns}$. Since, in the current study, I am not interested in the dynamics, a simple temperature rescaling every 100th step is used to hold the temperature at $293 \mathrm{~K}$. A time step of $1 \mathrm{fs}$ is used throughout this chapter, and periodic boundary conditions are applied in all directions. The measurements are taken every $10 \mathrm{fs}$ during the total simulation time of $16 \mathrm{~ns}$ for all models.

\section{Monte Carlo Simulation}

To validate the MD simulations with the double-exponent DI and DD models I have carried out MC simulations using the Metropolis algorithm with the Verlet neighbor list as described in Chap. 2. The maximal local displacement for each atom along any axis was $0.15 \AA$ so that the acceptance ratio was approximately $56 \%$ and $73 \%$ for the double-exponent DI and DD models, respectively. Initially, the $\mathrm{Hg}$ film is equilibrated for $3 \times 10^{4} \mathrm{MC}$ sweeps, where each of the $2 \times 10^{3}$ atoms was attempted to move once 
in $1 \mathrm{MC}$ sweep. Following, the density profiles were averaged over $1.045 \times 10^{6}$ and $5.35 \times 10^{5}$ sweeps for the double-exponent DI and DD models, respectively.

\subsubsection{Results}

\section{Density profiles and surface tension}

Fig. 3.2 shows the density profiles for the DI, DD, EAM2013 and EAM2006 models of liquid mercury at $T=293 \mathrm{~K}$ as obtained from my simulations. One can see that the DI model produces weak surface density oscillations (as seen previously at different temperatures [122]) but, at the same time, it yields the correct experimental bulk coexistence density, $\rho_{\text {coex }}$, of $13550 \mathrm{~kg} / \mathrm{m}^{3}$ [145], whereas the DD model yields the wrong coexistence density of around $10060 \mathrm{~kg} / \mathrm{m}^{3}$ with somewhat stronger surface density oscillations. As expected, the density profiles from the MD and MC simulation overlay perfectly for the DI and DD models, respectively. Both, the EAM2013 and EAM2006 models, give values of $\rho_{\text {coex }}$ of 13250 and $13180 \mathrm{~kg} / \mathrm{m}^{3}$, respectively. These values are both very close to the above mentioned experimental value of $\rho_{\text {coex }}$. The corresponding density profiles of both EAM models posses strongly oscillating features (Fig. 3.2).

The surface tension, $\gamma$, was calculated with the help of the Kirkwood-Buff relation [146, 147] in the course of the MD simulations as

$$
\gamma=\frac{1}{2} \int d z\left(p_{n}-p_{\tau}\right)
$$

where $p_{n}=p_{z z}$ and $p_{\tau}=\left(p_{x x}+p_{y y}\right) / 2$ are normal and tangential (w.r.t. the liquid $\mathrm{Hg}$ film's surface) stresses given by the diagonal elements of the stress tensor $p_{x x}, p_{y y}$ and $p_{z z}$. Since there are two surfaces (on the top and bottom of the $\mathrm{Hg}$ film), there is the factor of $1 / 2$ in front of Eq. 3.17. For the on-the-fly numeric calculation of $\gamma \mathrm{I}$ have used the discretization scheme of Eq. 3.17 described in Ref. [135]. My simulations yielded the values of $\gamma=0.18,0.23,0.306$ and $0.31 \mathrm{~N} / \mathrm{m}$ for the DI, DD, EAM2013 and EAM2006 models, respectively. One sees that the EAM models give the closest agreement with the experiment value of the surface tension of the bare surface of liquid mercury. Despite of the lowest value of $\gamma$ from the DI model, it represents a potential interest (because of its thermodynamic robustness and computational efficiency) to optimize this model in order to achieve a better agreement with experiments. I am going to explore this 


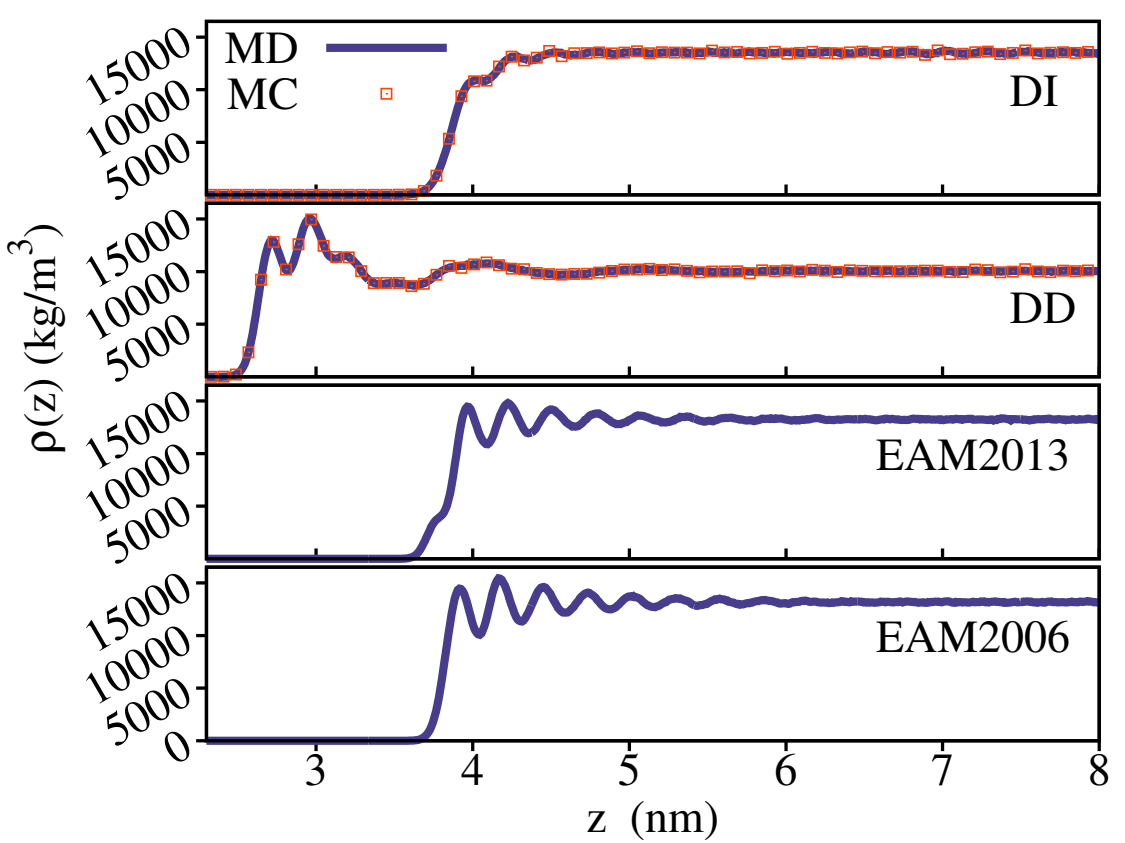

Figure 3.2: Density profiles for the studied atomistic models of the liquid mercury at $T=293 \mathrm{~K}$.

possibility in the next section (Sec. 3.2).

\section{Free energy}

In order to deepen our physical understanding of the influence of the density dependence of the studied mercury models on the phase behavior I employ the liquid-state theory (LST) [90, 92, 94] explained in Sec. 2.3. I start by discussing the Helmholtz free energies (Eq. 2.40) for the DI and DD models, which are shown in Fig. 3.3. The striking distinction of the DI model compared to the DD one is that the DI model features a single region of negative curvature of $F(\rho)$. This curvature region corresponds to the spinodal region inside the liquid-vapor miscibility gap. The DD model, on the contrary, reveals two regions of negative curvature divided by the metastable region in the density range from $8000 \mathrm{~kg} / \mathrm{m}^{3}$ to $11000 \mathrm{~kg} / \mathrm{m}^{3}$. Precisely here is the DD model density-dependent in contrast to the DI model (recall Eq. 3.4). In the following we are going to convince ourselves that such behavior of the free energy of liquid mercury from the DD model dramatically alters the overall phase behavior. As mentioned above, if two thermodynamic states coexist with each other, one can determine their respective 


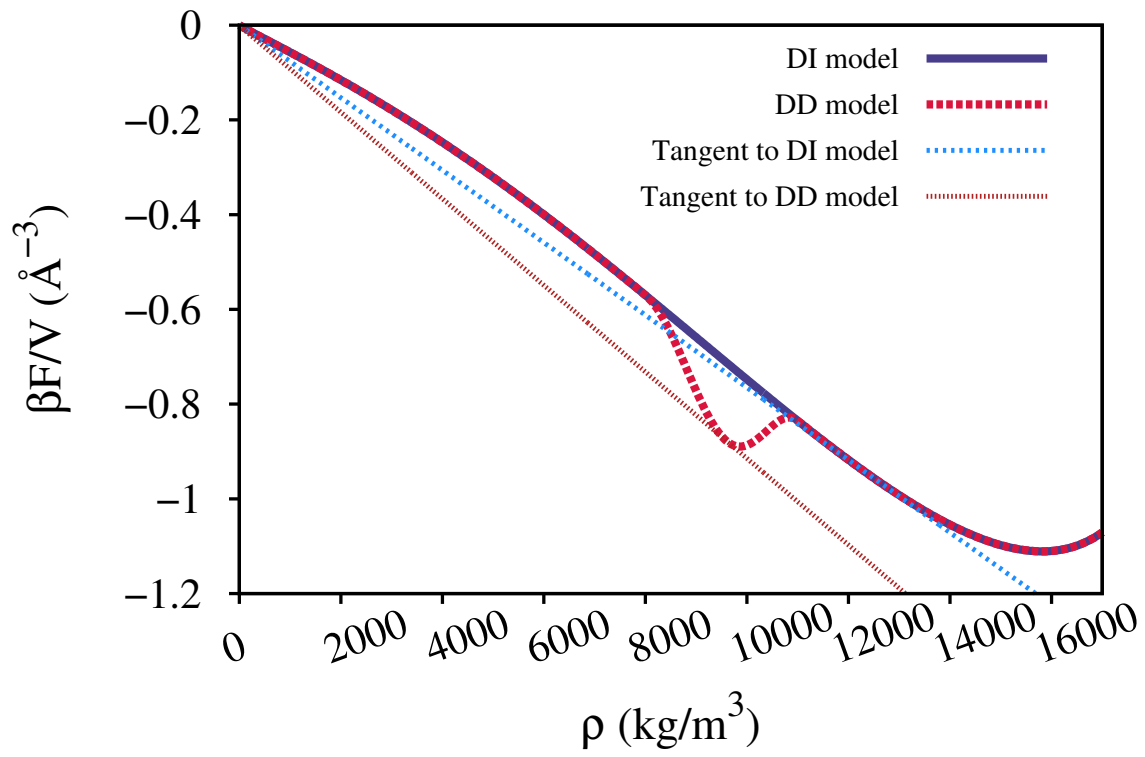

Figure 3.3: Free energy for the DI (dark blue solid line) and DD (red dashed line) models. Straight lines present the double tangents to the free energy of the DI (blue dashed line) and DD (dark red double short dashed line) models.

densities from the double tangent construction to the free energy versus density curve (see e.g. Ref. [96]), because both coexisting phases must have the same pressure and chemical potential values. This means basically that the points, at which the double tangent contacts the free energy curve, define two systems (and the densities thereof), which can coexist with each other.

As one can infer from Fig. 3.3, the coexistence density $\rho_{\text {coex }}$ of liquid mercury with its vapor is $12320 \mathrm{~kg} / \mathrm{m}^{3}$ and $9560 \mathrm{~kg} / \mathrm{m}^{3}$ for the DI and DD models, respectively. Of course, my LST results are very approximate by virtue of the simple approximations invoked by the LST approach. Nevertheless, they allow to gain a clear understanding of what happens when one inserts a density dependence in the DI pair potential on an ad-hoc basis. Namely, the additional dip in the free energy for the DD model with its minimum in the vicinity of $10000 \mathrm{~kg} / \mathrm{m}^{3}$ inhibits the coexistence of liquid mercury at a higher density of $13550 \mathrm{~kg} / \mathrm{m}^{3}$ with its vapor. As a result we observe two coexistence regions. The first coexistence region corresponds to the coexistence of a $\mathrm{Hg}$ vapor with the low-density liquid of about $9560 \mathrm{~kg} / \mathrm{m}^{3}$. And the second coexistence region cor- 


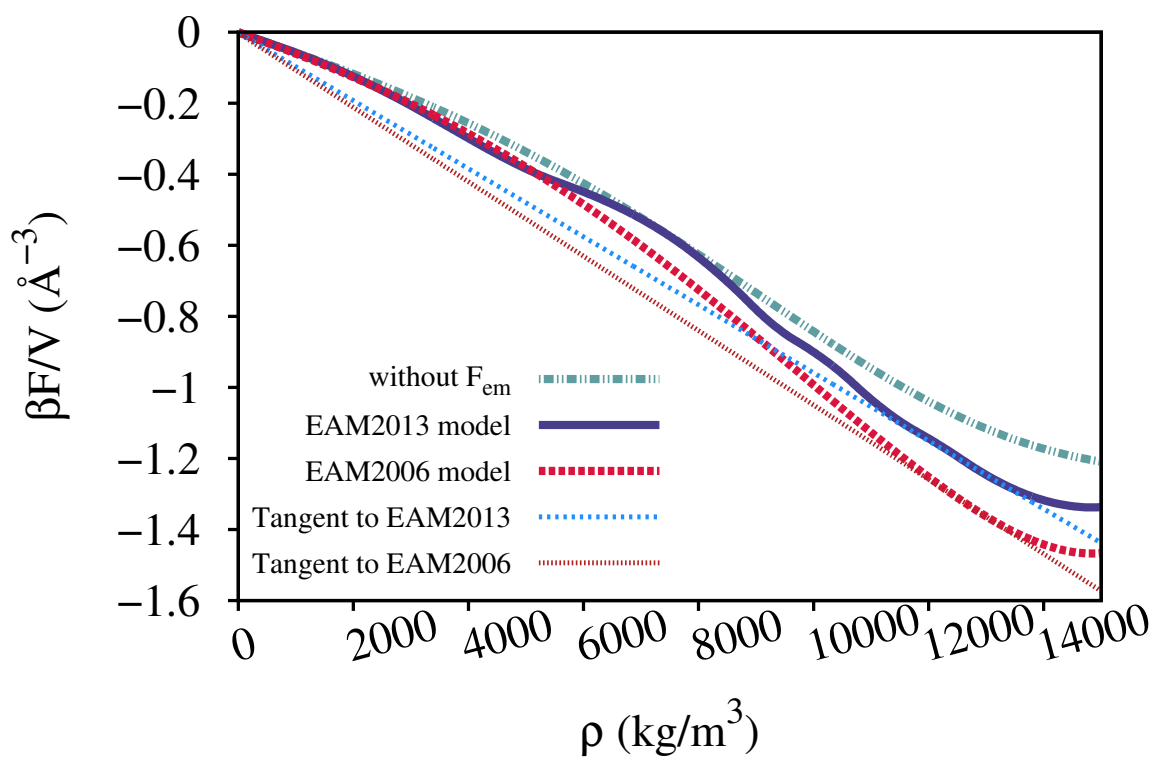

Figure 3.4: Free energy for the embedded-atom models of mercury.

responds to the spurious liquid-liquid phase coexistence between liquids of density of about $10000 \mathrm{~kg} / \mathrm{m}^{3}$ and $15000 \mathrm{~kg} / \mathrm{m}^{3}$. I expect that in the simulations the latter miscibility gap may be preempted by the coexistence of liquid mercury and its crystalline phase. The above LST results rationalize my MD simulations of the DI and DD models.

On the contrary, as Fig. 3.4 shows, the LST free energy curves for the EAM2013 and EAM2006 models have no spurious features that might dramatically alter the phase behavior. It should be noted that the free energy for the EAM2013 model reveals some regions of positive curvature for $\rho<13000 \mathrm{~kg} / \mathrm{m}^{3}$. This indicates a number of possible metastable coexistence regions at low densities (Fig. 3.4). But as the MD simulations indicate, these possible coexistences at lower densities are preempted by the liquidvapor coexistence at the density of liquid mercury of about $13000 \mathrm{~kg} / \mathrm{m}^{3}$. Therefore, the form of $U_{e m}$ of the EAM2013 model does not change the thermodynamics of phase coexistence nor I see a peculiar density profile of the mercury film. The free energy of the EAM models without the contributions of the respective embedded energies is shown in Fig. 3.4 for the comparison as well. From the double-tangent construction I obtain $\rho_{\text {coex }}=12800 \mathrm{~kg} / \mathrm{m}^{3}$ for the EAM2006 model. Taking into account the simplicity of the LST approximations, the agreement of the LST predictions with the results of the 
atomistic MD simulations appears to be very good. The effective hard sphere diameter (Eq. 2.44 for both EAM models equals to $2.96 \AA$ and is independent of density.

\subsection{Optimized model for liquid mercury}

\subsubsection{Derivation}

As discussed in my previous article [89], it is possible to optimize the DI model, which will allow to obtain higher surface tension, $\gamma$, and preserve the experimental value of $\rho_{\text {coex }}$ of liquid mercury. This is achieved by increasing the strength of the pair interaction, $A_{1}$, as is seen in the inset in Fig. 3.5. Simultaneously one has to change the parameter $b$ in order to preserve the coexistence density of the liquid phase. In this way we decrease the range of the attractive part of the pair interaction as shown in Figs. 3.5 and 3.6. As is discussed by Frenkel et al. [88], the range of the attractive interaction determines the stability of a liquid phase. As we shall see, a shorter range of attractive interactions and its stronger strength tend to shift the liquid-crystal phase boundary towards a lower density value for a given temperature. This is why there is a limit on how much one can decrease the range of attraction and increase its strength before crystallization sets in.

From Fig. 3.5 one can see that the increase of $A_{1}$ and $b$ corresponds to the increase of the hard core diameter $d$ (see Eq. 2.44) of mercury. This in turn corresponds to the increase of the packing fraction $\eta$ (Eq. 2.43). My best values for the optimized DI (ODI) parameters are $A_{1}=2.233335535 \mathrm{kcal} / \mathrm{mol}$ and $b=0.891 \AA^{-2}$. This yields $\eta=0.643$, which is slightly below 0.65 for the random closest packing of hard spheres [148]. If one increases $A_{1}$ any further at $T=293 \mathrm{~K}$, the system would be inevitably driven into the crystalline phase.

The packing fraction $\eta$ for the ODI as well as for the LJ model in the liquid phase is higher than that of hard spheres at the liquid-solid transition. Using Eq. 2.44 I find $d=1.07 \sigma_{0}$ for the $\mathrm{LJ}$ fluid phase at the triple point. This yields the packing fraction $\eta=0.51$. For the hard-sphere fluid at the liquid-solid transition $\eta=0.494$ [148].

The values of surface tension $\gamma$ for the ODI (purple solid line in Fig. 3.6) and intermediate (red dashed line in Fig. 3.6) sets of the optimized parameters are around 0.27 $\mathrm{N} / \mathrm{m}$ and $0.21 \mathrm{~N} / \mathrm{m}$, respectively. These values are still smaller than the experimental 


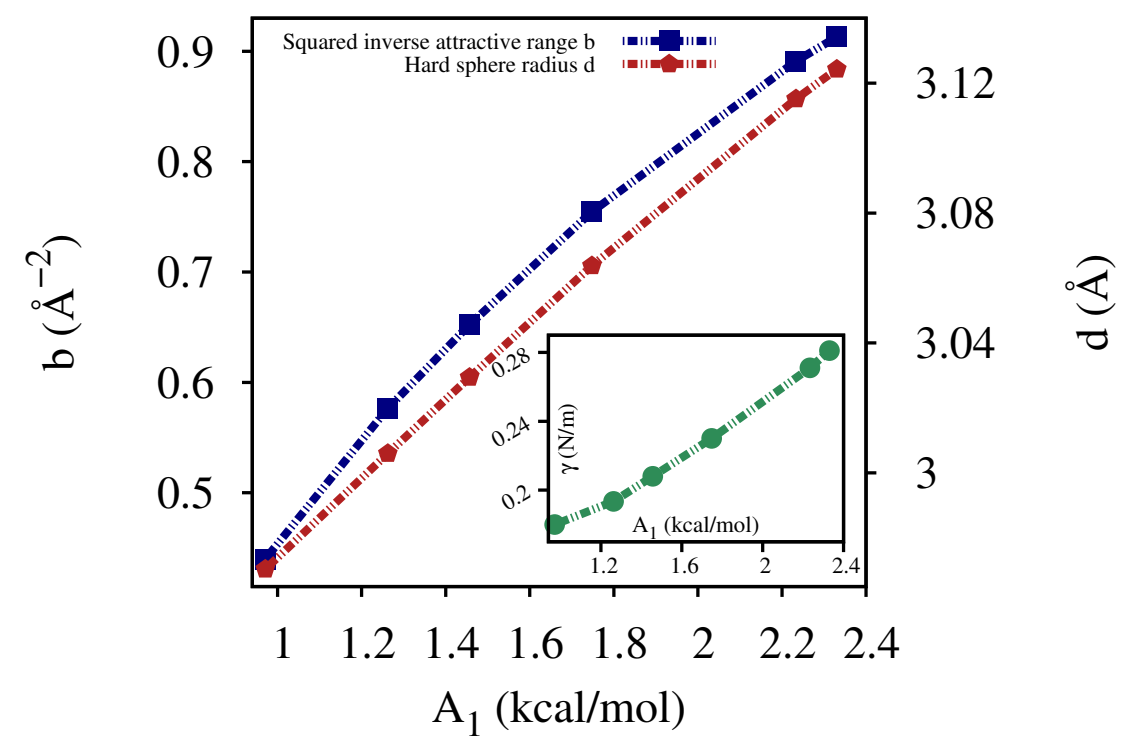

Figure 3.5: The squared inverse attractive range $b$ (left y-axis, dark blue squares), the hard core radius $d$ (right y-axis, dark red pentagons) and the surface tension $\gamma$ (inset) are presented as functions of the strength of interaction, $A_{1}$. Density in the middle of the $\mathrm{Hg}$ film differs from the experimental one by about $0.1 \%$ for each pair of $\left(A_{1}, b\right)$ values. Values of $d$ and $\gamma$ at given $A_{1}$ correspond to the respective value of $b$. Lines connecting the data points serve as guides to eyes.

value but, nevertheless, larger than the $\gamma$ value for the original DI model. In order to increase $\gamma$ further one would have to add an additional long-range term to the DI pair potential. This would allow to keep the range of attraction large enough in order to prevent the crystallization of the system.

The density profiles obtained from the simulations with the DI, intermediate, and the ODI models are represented in Fig. 3.7. In all the cases the densities of liquid $\mathrm{Hg}$ at the coexistence with its vapor agree very well with the experimental bulk density of $13550 \mathrm{~kg} / \mathrm{m}^{3}$ for liquid $\mathrm{Hg}$ at $T=293 \mathrm{~K}$ [145] deviating from it only by around $0.1 \%$. The ODI model shows much stronger surface oscillations at the liquid-vapor interface compared to the DI model (Fig. 3.7). This suggests that the higher values of $\gamma$ imply stronger layering at the mercury surface. Though the 1 st outer peak in the density profile of the ODI model is smaller than the 2nd one (as opposed to experiment [98]), it should not be significant for the construction of coarse-grained models from my 




Figure 3.6: Comparison of the density-independent model with different values of $A_{1}$ and $b$ parameters, that yield the same bulk density.

atomistic ODI force field, because the large-scale properties such as contact angle of a drop are mainly determined by the interfacial tension. Moreover, the surface tension of the ODI model is comparable (very close) to the EAM models (see the previous section for the comparison).

Finally, I note that when no external fields are applied the local position of the $\mathrm{Hg}$ surface is influenced mainly by capillary waves with the mean square amplitude [149]

$$
a_{\mathrm{cw}}^{2}=\frac{k_{\mathrm{b}} T}{2 \pi \gamma} \ln (L / l)
$$

where $L$ is the lateral size of the system, $l$ is the size of the mercury atom (i.e. a short wavelength cut-off of the capillary wave spectrum). I use the hard sphere diameter, $d$, of mercury as a measure for $l$. Giving Eq. 2.44, $d$ is readily estimated for the ODI model and equals to $3.12 \AA$. I use this value of $d$ together with $\gamma=0.27 \mathrm{~N} / \mathrm{m}, T=293 \mathrm{~K}$ and $L=24.56 \AA$ in the Eq. 3.18 in order to obtain $a_{c w}^{2}=0.49 \AA$. This means that the local shape of the $\mathrm{Hg}$ film deviates on average by about $\pm 0.7 \AA$ from the planar geometry. On the atomistic scale this is an insignificantly small deviation, because it is noticeably 
smaller than the diameter of a mercury atom. Hence, I anticipate that the influence of the capillary waves on the interface profiles and surface tension for the lateral system sizes (used in this chapter) is also insignificant.

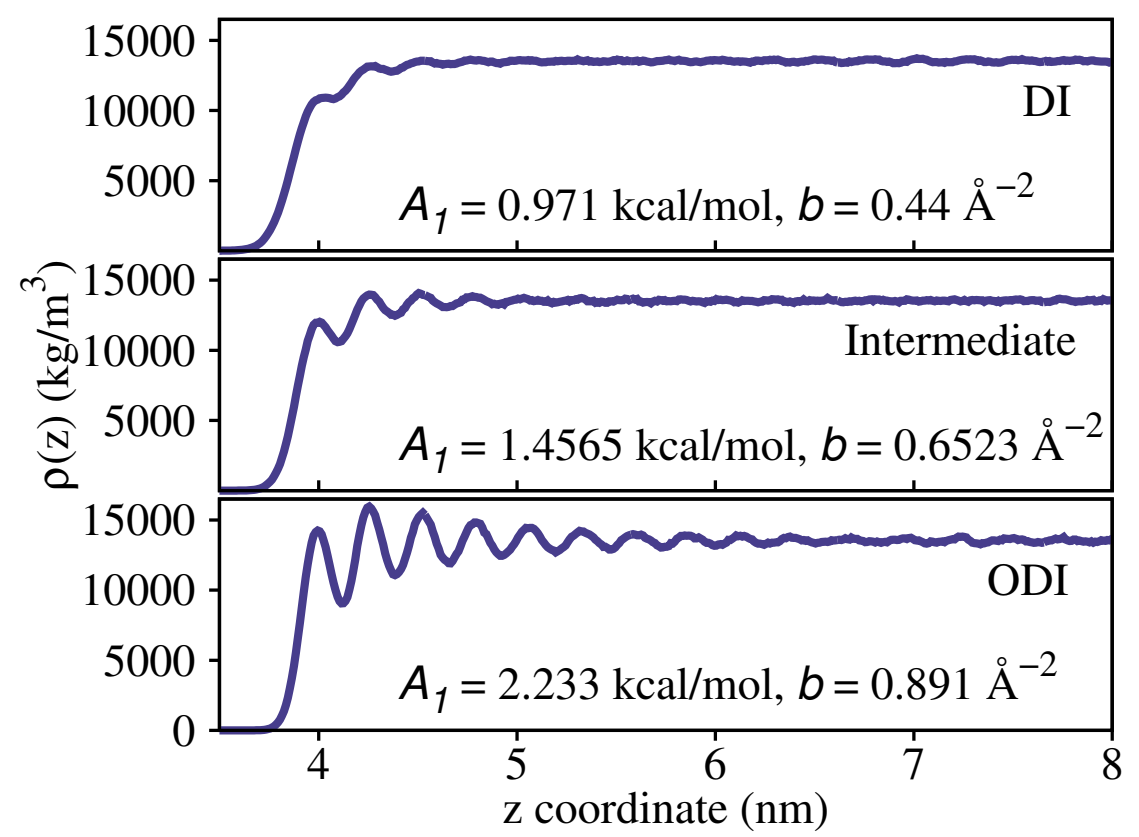

Figure 3.7: Density profiles of the density-independent double exponent model (Eq. 3.3 for various sets of $A_{1}$ and $b$ parameters that yield the same bulk density of $13550 \mathrm{~kg} / \mathrm{m}^{3}$.

\subsubsection{Validation}

\section{Temperature behavior}

To compliment the discussion of the ODI potential I have calculated the $\rho-T$ phase diagrams of mercury from the MD simulations and from the double-tangent construction to the LST free energy curves. The results are depicted in Fig. 3.8, The calculated phase diagrams of liquid $\mathrm{Hg}$ basically serve two purposes. First, I study the temperature behavior of the ODI model in order to understand, up to what temperature the ODI model provides a satisfactory description of the mercury phase diagram, so that one does not have to re-optimize parameters $A_{1}$ and $b$. Second, these results help us to better estimate the limitations of the LST approach compared to the MD simulations. As one sees from Fig. 3.8, the vapor branches of both phase diagrams perfectly coincide with 


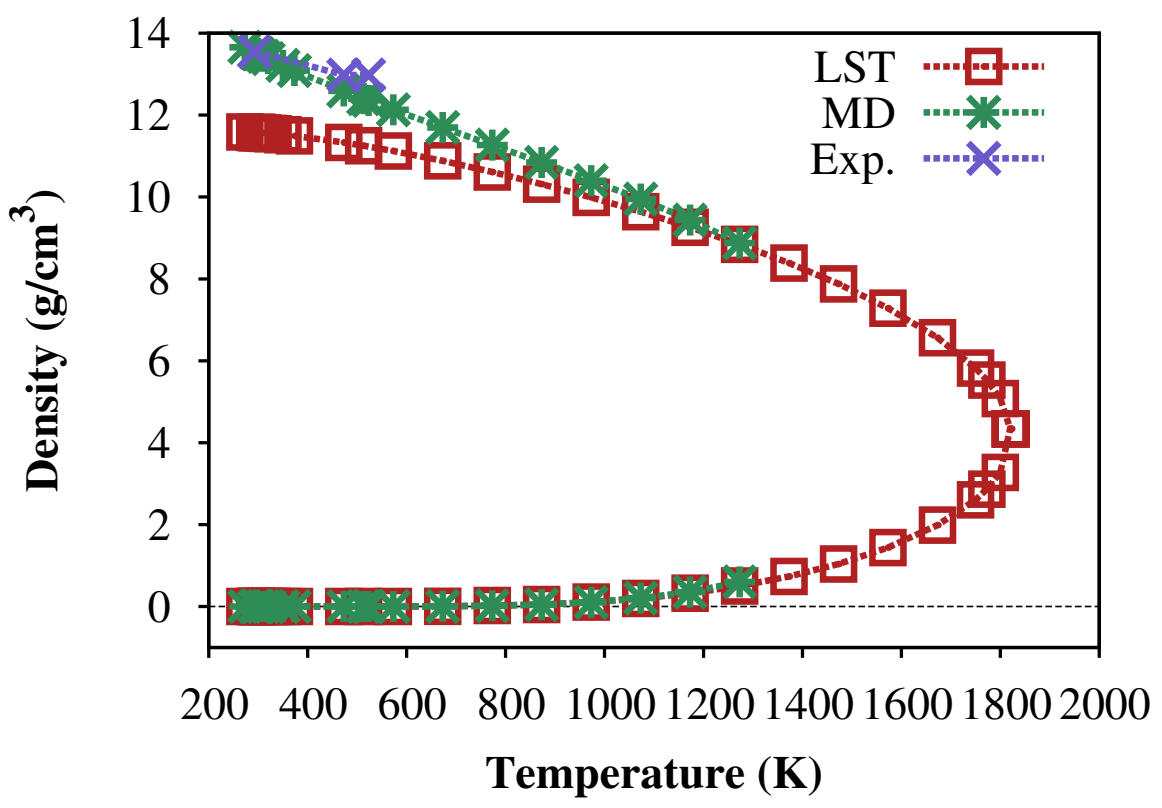

Figure 3.8: Comparison of the $\rho-T$ phase diagrams of mercury obtained by my MD simulations of the ODI model (green stars) and by the double tangent construction to the LST free energy curves (red squares). Experimental values of the density of liquid mercury at relevant temperatures are also shown (purple crosses) [145].

each other. We also see that the LST matches quite well the MD liquid branch starting from about $1200 \mathrm{~K}$. My MD simulations yield at $T=523 \mathrm{~K}$ the bulk density of liquid mercury of $12.4 \mathrm{~g} / \mathrm{cm}^{3}$ at the coexistence with its vapor, which differs just by $4.5 \%$ from the experimental one at the same temperature [145]. As the temperature increases the deviation from the experimentally observed coexistence densities will grow. Despite this, I note that such high temperatures $(>523 \mathrm{~K})$ lie above the typical applicability temperature range for the organo-metallic surfaces, since at such temperatures mercury features coexistence of a vapor phase of a non-negligible density with a liquid phase. Moreover, at $T=373 \mathrm{~K}$ a thiol monolayer on a crystalline surface would become very distorted [150]. Furthermore, we will see in one of the following chapters that a well ordered crystalline phase of octadecanethiol molecules becomes completely destroyed already at $T=350 \mathrm{~K}$. Giving these results as well as the ones for the surface tension, one can conclude that the ODI model describes the surface of liquid $\mathrm{Hg}$ quite satisfactory in the range of temperatures of practical interest. In principle, the above optimization procedure can be applied at any desired temperature in order to improve the precision. 


\section{Bulk properties}

In order to finalize the discussion of the ODI model I have also evaluated bulk properties of liquid $\mathrm{Hg}$. For this purpose a cubic bulk systems with side length of $36.6351 \AA$ and $N=2 \times 10^{3} \mathrm{Hg}$ atoms was used. Such bulk properties of the liquid mercury as the specific heat at constant volume, $C_{V}$, and constant pressure, $C_{P}$, were evaluated. These quantities are defined as follows (see e.g. [75])

$$
\begin{aligned}
& C_{V}=(d U / d T)_{V}, \\
& C_{P}=(d U / d T)_{P} .
\end{aligned}
$$

In order to compute $C_{V}$ and $C_{P}$ the simulations were performed at temperatures of 290.5, 293, 295.5 and $298 \mathrm{~K}$. For the calculation of $C_{P}$ and of the heat of evaporation (see below) the pressure was held constant at 0 atm by using the Berendsen barostat [78] with the pressure damping parameter of 1 ps. Following, the system total energy, $U$, is differentiated with respect to temperature by using a four-point finite difference formula for the 1st derivative in order to obtain the final values of $C_{V}$ and $C_{P}$ for $T=293 \mathrm{~K}$. My simulations yield 22.67 and $29.86 \mathrm{~J} /(\mathrm{mol} \mathrm{K})$ for $C_{V}$ and $C_{P}$, respectively. Giving the available experimental data for $T=293 \mathrm{~K}$ and $P=1 \mathrm{~atm}[151]$ we can see that the ODI result for $C_{V}$ is slightly below the experimental value of $24.137 \mathrm{~J} /(\mathrm{mol} \mathrm{K})$, whence the ODI result for $C_{P}$ is slightly above the experimental value of $27.9 \mathrm{~J} /(\mathrm{mol} \mathrm{K})$.

In addition, I have computed the heat of evaporation, $\Delta H$, (see e.g. [152])

$$
\Delta H=\Delta U_{p o t}+R T,
$$

where $\Delta U_{p o t}$ is the change of the system's potential energy per mole upon evaporation at constant pressure and $R$ is the gas constant. I obtain $\Delta U_{\text {pot }}=62.22 \mathrm{~kJ} / \mathrm{mol}$, which, finally, yields $\Delta H=64.66 \mathrm{~kJ} / \mathrm{mol}$. This value of $\Delta U_{p o t}$ lies within $4.9 \%$ of the experimental one of $61.64 \mathrm{~kJ} / \mathrm{mol}[151]$.

I consider the ODI estimates of the bulk properties of liquid $\mathrm{Hg}$ to be quite satisfactory, especially giving that the ODI model was devised primarily with an intention to obtain a computationally efficient force field for the liquid mercury that produces a strongly oscillating $\mathrm{Hg}$ surface of high tension in order to serve as a support in simulations of organic molecular monolayers. 


\subsection{Discussions and conclusions}

In the present chapter a number of previously proposed force fields for the molecular simulation of liquid mercury has been evaluated in the ability to model a strongly oscillating density profile of a liquid mercury surface and its high tension. It is found that the embedded-atom models yield the best agreement with the experiment value of the surface tension as well as strongly stratified density profiles at room temperature. On the contrary, the density-independent double-exponent model produced a too low surface tension and weakly oscillating density profile for the liquid mercury surface at the desired temperature. Nevertheless, this model is thermodynamically robust, because it is capable of producing the right coexistence densities of liquid mercury, and, generally speaking, is much more computationally efficient compared to any density-dependent model including the embedded-atom ones. This is why this density-independent model is optimized to improve the agreement of the surface tension value with experiment and to yield the highly oscillating density profile of liquid $\mathrm{Hg}$ at room temperature. The so obtained value of the surface tension is comparable with ones from the simulations with the embedded-atom models. The optimization procedure is highlighted in detail and may be repeated for any desired temperature. Additionally, it is shown that the optimized parameters of the density-independent model yield a quite satisfactory description of the surface of liquid mercury for the temperature region of practical interest (i.e. up to 500-600 K). One way to improve further the characteristics of liquid mercury in a computer simulation is to try to re-parametrize the embedded-atom models according to the recently obtained experimental data on the equation of state of the liquid mercury [153]. Another possibility consists in adopting the modified embedded-atom method, where a non-uniform atomic distribution near to the surface might be explicitly taken into account [154]. As an alternative to this, for the derivation of a new force field for liquid mercury one could try a newly developed scheme for the systematic modification of the embedded-atom models, which allows for an implicit account of anisotropy of density distributions [155]. It has been also shown that the semi-analytic liquid-state theory is an easy and immediate tool for a qualitative scan of coexistence behavior of density-dependent force fields for molecular simulations. In the next chapter the optimized density-independent model will be now applied for the large-scale simulation of the alkylthiol self-assembly on the surface of liquid mercury. 


\title{
4 | Molecular coverage induced self- assembly of thiols on liquid mer- cury
}

\begin{abstract}
After developing the force field for the effective numerical simulation of the bare surface of liquid mercury in the previous chapter one is now fully equipped to make the next step. In this chapter the phase behavior of thiols as a function of the surface coverage will be investigated in detail. The experimentally observed coexistence of the molecules in the laying-down conformations with the crystalline phases of standing thiols has been attained in the simulations presented below. The structure in the crystalline thiol phases is carefully analyzed. In order to adequately capture the effect of the surface coverage one has to simulate extremely large systems, which drastically increases computational costs. This is why a smart way of initializing the surfactant systems is crucial in order to reduce equilibration times. In the beginning of this chapter the molecular model and the force fields, which were used in the current study, are introduced. Special attention is devoted to the equilibration procedure. Following, I proceed with the description of the obtained results. The simulations presented in the current chapter allow for the first time to directly access the molecular-scale structure of the self-assembled alkylthiol monolayers on the surface of liquid mercury and to understand factors that influence it. Most of the results on the equilibrium structure of the thiol SAMs from this chapter the reader can also find in my recent publication [156].
\end{abstract}




\subsection{Simulation details}

\subsubsection{Force fields}

In order to simulate an alkylthiol monolayer on a liquid mercury surface I have utilized a united atom (UA) representation of the alkyl $\left(\mathrm{CH}_{2}\right)$ and methyl $\left(\mathrm{CH}_{3}\right)$ groups, in which hydrogens $(\mathrm{H})$ are not explicitly treated and $\mathrm{CH}_{2}$ and $\mathrm{CH}_{3}$ groups are modeled as single particles, which allows to considerably save computational time and simulate systems of more realistic sizes. Mercury and sulfur (S) atoms are treated atomistically. Fig. 1.7 shows a sketch of such molecular model. A linear chain of alkyl groups together with sulfur head- and methyl endgroups comprises a single alkylthiol molecule. From valance considerations I have chosen to model an ideal situation when two alkylthiol molecules are chemisorbed onto a single mercury atom. There have been also experimental evidences in favor of this choice [64]. Thus, a single surfactant molecule is represented by the complex of two thiols bound to one $\mathrm{Hg}$ atom $(\mathrm{R}-\mathrm{S}-\mathrm{Hg}-\mathrm{S}-\mathrm{R})$ as shown in Fig. 1.7 A. As previously discussed the mercury atom that has chemical bonds connecting it to a thiol molecule will be dubbed a bound mercury and designated as $\mathrm{Hg}^{*}$, whereas bulk mercury atoms shall be denoted simply as $\mathrm{Hg}$.

The total energy of such molecular system consists of bonded and non-bonded contributions

$$
U_{\text {tot }}=U_{\text {bonded }}+U_{\text {non-bonded }} \text {. }
$$

The bonded part describes all intramolecular interactions due to elastic bonds, $U_{b o n d}$, and bends, $U_{\text {bend }}$, as well as due to torsional degrees of freedom, $U_{\text {torsion }}$,

$$
U_{\text {bonded }}=U_{\text {bond }}+U_{\text {bend }}+U_{\text {torsion }}
$$

The bond energy, $U_{b o n d}$, is given by the sum over all covalent bonds in the system

$$
U_{b o n d}=\sum_{m=1}^{N_{m}^{t o t}} \sum_{i=1}^{N_{a}-1} U_{r}\left(r_{m i}\right)
$$

where $N_{m}^{t o t}$ and $N_{a}$ are the total number of surfactant molecules and atoms per molecule, respectively. In the above equation $r_{m i}$ is the length of the $i$-th bond belonging to the 
$m$-th surfactant molecule. The elastic bond stretching is given by harmonic springs

$$
U_{r}(r)=K_{r}\left(r-r_{0}\right)^{2}
$$

The respective spring constants and equilibrium bond distances are given in Tab. 4.1

\begin{tabular}{l||l|l||l|l} 
Bond type & $K_{r}\left(\mathrm{kcal} /\left(\mathrm{mol} \AA^{2}\right)\right)$ & Ref. & $r_{0}(\AA)$ & Ref. \\
\hline \hline $\mathrm{CH}_{2,3}-\mathrm{CH}_{2}$ & 95.899 & {$[157]$} & 1.53 & {$[157]$} \\
\hline $\mathrm{CH}_{2}-\mathrm{S}$ & 222.0 & {$[33]$} & 1.82 & {$[39]$} \\
\hline $\mathrm{S}-\mathrm{Hg}$ & 222.0 & & 2.305 & {$[158]$}
\end{tabular}

Table 4.1: Parameters for the bond energy (Eq. 4.4 for various bond types.

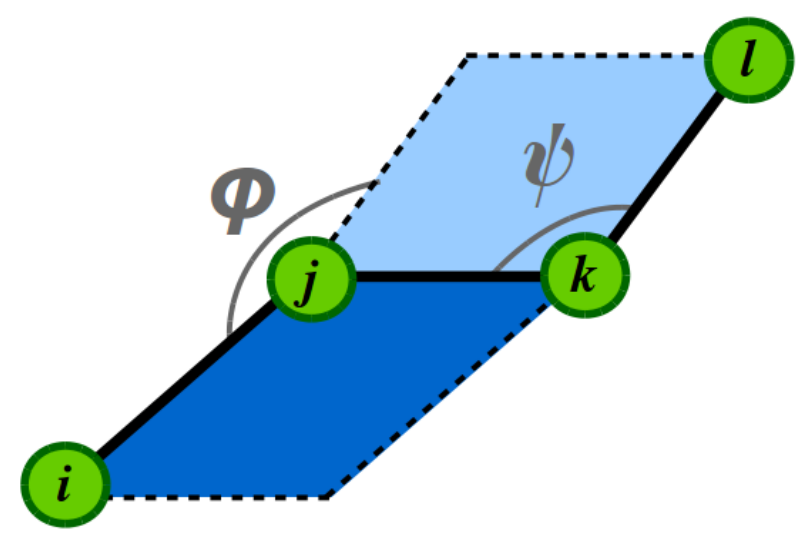

Figure 4.1: A segment of a surfactant chain depicting adjacent atoms $i, j, k$ and $l$. The bonds between the adjacent atoms are modeled as harmonic springs (Eq. 4.4). The degrees of freedom associated with a bend angle, $\psi$, formed by two neighboring bonds are also described via harmonic springs (Eq. 4.6). The angle $\phi$ defines the torsional rotation around the bond between atoms $j$ and $k$. The respective torsional degrees of freedom are modeled by a multiharmonic potential (Eq. 4.8).

The bending energy is given by the sum of the contributions of all the molecular angles in the system

$$
U_{\text {bend }}=\sum_{m=1}^{N_{m}^{t o t}} \sum_{i=1}^{N_{a}-2} U_{\psi}\left(\psi_{m i}\right),
$$

where $\psi_{m i}$ is the $i$-th angle of the $m$-th molecule defined as an angle between two bonds formed by three adjacent atoms of the same molecule (see Fig. 4.1). Analogical to the 


\begin{tabular}{l||l|l||l|l} 
Bend angle & $K_{\psi}\left(\mathrm{kcal} /\left(\mathrm{mol} \mathrm{rad}^{2}\right)\right)$ & Ref. & $\psi_{0}(\mathrm{Deg})$ & Ref. \\
\hline \hline $\mathrm{CH}_{2,3}-\mathrm{CH}_{2}-\mathrm{CH}_{2}$ & 62.100128125 & {$[159]$} & $114.4^{\circ}$ & {$[159]$} \\
\hline $\mathrm{S}-\mathrm{CH}_{2}-\mathrm{CH}_{2}$ & 62.5 & & $114.4^{\circ}$ & {$[46]$} \\
\hline $\mathrm{CH}_{2}-\mathrm{S}-\mathrm{Hg}$ & 47.1 & {$[158]$} & $101.1^{\circ}$ & {$[158]$} \\
\hline $\mathrm{S}-\mathrm{Hg}-\mathrm{S}$ & 26.0 & {$[158]$} & $178.6^{\circ}$ & {$[158]$}
\end{tabular}

Table 4.2: Parameters for the bending energy (Eq. 4.6) for various types of molecular angles.

elastic bond energy, the bending energy, $U_{\psi}$, is modeled by harmonic springs

$$
U_{\psi}=K_{\psi}\left(\psi-\psi_{0}\right)^{2}
$$

where the values of $K_{\psi}$ and $\psi_{0}$ for various molecular angles are given in Tab. 4.2. The torsional energy is defined as a sum of the contribution from all torsion angles in the system

$$
U_{\text {torsion }}=\sum_{m=1}^{N_{m}^{t o t}} \sum_{i=1}^{N_{a}-4} U_{\phi}\left(\phi_{m i}\right),
$$

where $\phi_{m i}$ is the $i$-th torsional angle of the $m$-th molecule. A torsional angle describes the rotation of a molecular backbone around a given bond vector, $\mathbf{r}_{n, n+1}=\mathbf{r}_{n}-\mathbf{r}_{n+1}$, between two adjacent atoms, $n$ and $n+1$, of the same molecule (see Fig. 4.1). Such angle is then defined as an angle between two planes, each built by the pair of vectors $\left(\mathbf{r}_{n, n+1}, \mathbf{r}_{n+1, n+2}\right)$ and $\left(\mathbf{r}_{n-1, n}, \mathbf{r}_{n, n+1}\right)$, respectively. Typically, if one takes into account all bond vectors in the molecule, the number of torsion angles in a simple chain molecule is $N_{a}-3$. In our case, the $\mathrm{S}-\mathrm{Hg}-\mathrm{S}$ angle is very close to $180^{\circ}$ and, consequently, it is difficult to define separate rotations around two adjacent $\mathrm{S}-\mathrm{Hg}$ bonds [158]. This is why, instead of these two rotations, I use a single one around $\mathrm{S}-\mathrm{Hg}-\mathrm{S}$ segment in my simulations similar to Ref. [158]. Hence, the second summation in Eq. 4.7 goes up to $N_{a}-4$. I choose a single torsion contribution to Eq. 4.7 to be of a multiharmonic type (see e.g. Refs. [77, 156, 159, 160]), which has a very flexible functional form

$$
U_{\phi}(\phi)=\sum_{i=1}^{5} A_{i} \cos ^{i-1} \phi,
$$

the values of the respective parameters $A_{i}$ for various torsion types are given in Tab. 4.3. 


\begin{tabular}{l||l|l} 
Torsion type & $A_{i}(\mathrm{kcal} / \mathrm{mol})$ & Ref. \\
\hline \hline $\mathrm{CH}_{2,3}-\mathrm{CH}_{2}-\mathrm{CH}_{2}-\mathrm{CH}_{2}$ & $A_{1}=2.007115885082$ & {$[159]$} \\
\hline equivalent to the OPLS & $A_{2}=-4.0122147580025$ & {$[159]$} \\
\hline force field for alkanes & $A_{3}=0.271014895158$ & {$[159]$} \\
\hline (e.g. compare with Ref. [60]) & $A_{4}=6.2894890532754$ & {$[159]$} \\
\hline & $A_{5}=0.0$ & {$[159]$} \\
\hline \hline $\mathrm{S}-\mathrm{CH}_{2}-\mathrm{CH}_{2}-\mathrm{CH}_{2}$ & $A_{1}=2.007115885082$ & {$[39]$} \\
\hline & $A_{2}=-4.0122147580025$ & {$[39]$} \\
\hline & $A_{3}=0.271014895158$ & {$[39]$} \\
\hline \hline $\mathrm{CH}-\mathrm{CH}-\mathrm{S}-\mathrm{Hg}$ & $A_{4}=6.2894890532754$ & {$[39]$} \\
\hline fit by Eq. 4.8 of the & $A_{5}=0.0$ & {$[39]$} \\
\hline respective torsional & $A_{1}=0.5099999990$ & {$[158]$} \\
\hline energy from Ref. [158] & $A_{2}=-0.5700000000$ & {$[158]$} \\
\hline & $A_{3}=0.3000000110$ & {$[158]$} \\
\hline \hline $\mathrm{CH}_{2}-\mathrm{S}-\mathrm{S}-\mathrm{CH}{ }_{2}$ & $A_{4}=1.0800000000$ & {$[158]$} \\
\hline symmetrized version of & $A_{5}=-0.0000000108194621$ & {$[158]$} \\
\hline the respective potential & $A_{1}=-0.0889828674$ & \\
\hline from Ref. [158] & $A_{2}=-0.0405435305$ & \\
\hline & $A_{3}=0.8999657630$ & \\
\hline
\end{tabular}

Table 4.3: Parameters for the torsion energy (Eq. 4.8) for various types of torsion angles.

The non-bonded interaction describes intermolecular interactions, such as Van der Waals and metallic (for bulk $\mathrm{Hg}$ ) interactions, and is given by

$$
\begin{aligned}
U_{\text {non-bonded }}= & \sum U_{\mathrm{Hg}-\mathrm{Hg}}+\sum U_{\mathrm{Hg}-\mathrm{Hg}^{*}}+\sum U_{\mathrm{Hg}-\mathrm{S}}+\sum U_{\mathrm{Hg}-\mathrm{CH}_{2}}+\sum U_{\mathrm{Hg}-\mathrm{CH}_{3}} \\
& +\sum U_{S-\mathrm{S}}+\sum U_{\mathrm{Hg}^{*}-\mathrm{Hg}^{*}}+\sum U_{\mathrm{CH}_{2}-\mathrm{CH}_{2}}+\sum U_{\mathrm{CH}_{3}-\mathrm{CH}_{3}} \\
& +\sum U_{\mathrm{Hg}^{*}-\mathrm{S}}+\sum U_{\mathrm{Hg}^{*}-\mathrm{CH}_{2}}+\sum U_{\mathrm{Hg}^{*}-\mathrm{CH}_{3}} \\
& +\sum U_{S-\mathrm{CH}_{2}}+\sum U_{S-\mathrm{CH}_{3}}+\sum U_{\mathrm{CH}_{2}-\mathrm{CH}_{3}}
\end{aligned}
$$

where the summations run over all possible atom pairs of respective atom species.

For the pair interaction of the bulk mercury atoms, $U_{H g-H g}$, I use the optimized density-independent double-exponent force field derived in the previous section and also described in my paper [89]. Below I give its functional form once again for the 


\begin{tabular}{l||l|l|l|l|l|l} 
Type & $A_{0}(\mathrm{kcal} / \mathrm{mol})$ & $a(1 / \AA)$ & $A_{1}(\mathrm{kcal} / \mathrm{mol})$ & $b\left(1 / \AA^{2}\right)$ & $R_{0}$ & Ref. \\
\hline \hline $\mathrm{Hg}-\mathrm{Hg}$ & $190.1990928 \mathrm{E} 13$ & 12.48 & 2.233335535 & 0.891 & 3.56 & {$[89]$} \\
$\mathrm{Hg}-\mathrm{CH}_{2,3}$ & $190.1990928 \mathrm{E} 13$ & 12.48 & 0.5 & 2.0 & 3.56 & {$[156]$}
\end{tabular}

Table 4.4: Parameters for the $\mathrm{Hg}-\mathrm{Hg}$ and $\mathrm{Hg}-\mathrm{CH}_{2,3}$ interactions that follow the functional form of Eq. 4.10 .

sake of completeness

$$
U(r)=A_{0} \exp (-a r)-A_{1} \exp \left(-b\left(r-R_{0}\right)^{2}\right)
$$

The $\mathrm{UHg}_{\mathrm{Hg}} \mathrm{CH}_{2}$ and $\mathrm{UHg}_{\mathrm{Hg}}$ interactions follow the same functional form as Eq. 4.10 and were parametrized to yield an experimental adsorption energy of $5.4 \mathrm{~kJ} / \mathrm{mol}$ per alkyl group on liquid mercury [156]. The values of respective parameters from Eq. 4.10 for $\mathrm{Hg}-\mathrm{Hg}$ and $\mathrm{Hg}-\mathrm{CH}_{2,3}$ are summarized in Tab. 4.4 .

Since there is no available experimental data on thiol adsorption energies on liquid mercury, the interaction of bound and bulk $\mathrm{Hg}$ atoms among themselves, $\mathrm{U}_{\mathrm{Hg}-\mathrm{Hg}^{*}}$, as well as the interaction of the sulfur headgroup with the bulk mercury, $U_{H g-S}$, are free parameters of the model. These interactions are important for the magnitude of the adsorption energies, but should be of lesser importance for the lateral packing of the surfactants on the surface of liquid mercury. For the current study it is important to fulfill the condition of the chemisorption of thiols on liquid $\mathrm{Hg}$ surface, namely, when a thiol molecule is chemisorbed, it is unlikely to leave the surface of liquid mercury. This condition is easily satisfied by choosing the $U_{H g-H g^{*}}$ interaction equal to the bulk mercury interaction, $\mathrm{U}_{\mathrm{Hg}-\mathrm{Hg}}$. The interaction of bound $\mathrm{Hg}$ atoms with themselves, $U_{\mathrm{Hg}^{*}-\mathrm{Hg}^{*}}$, should not be as strong as of the bulk mercury, because the bound mercury is no longer metallic. This is why for the sake of simplicity this interaction as well as all respective cross-interactions (involving $\mathrm{Hg}^{*}$ ) and the $U_{\mathrm{Hg}-S}$ interaction are chosen to be the same as the $\mathrm{Hg}-\mathrm{CH}_{2,3}$ interaction.

The non-bonded interactions between thiol components, $\mathrm{US}_{\mathrm{S}-\mathrm{S}}, \mathrm{U}_{\mathrm{CH}_{2}-\mathrm{CH}_{2}}$ and $\mathrm{U}_{\mathrm{CH}_{3}-\mathrm{CH}_{3}}$, are modeled via the Lennard-Jones potential

$$
U_{L J}=4 \varepsilon\left[\left(\frac{\sigma_{0}}{r}\right)^{12}-\left(\frac{\sigma_{0}}{r}\right)^{6}\right]
$$


The values of $\varepsilon$ and $\sigma_{0}$ for various interaction types are given in Tab. 4.5 .

Fig. 4.2 shows the described above non-bonded potential used in my simulations. We see that the double-exponent potentials $\left(\mathrm{U}_{\mathrm{Hg}-\mathrm{Hg}}\right.$ and $\left.\mathrm{U}_{\mathrm{Hg}-\mathrm{CH}_{2,3}}\right)$ have a considerably shorter range compared to LJ-like models. For this type of interactions I use the cut-off radius, $r_{c}$, of $9 \AA$. For the LJ interactions $r_{c}$ is set to $15 \AA$. The short-ranged interaction of alkyl and methyl groups with the bulk mercury is very important in order to capture right phase behavior of alkanes on the surface of liquid mercury [156].

\begin{tabular}{l||l|l|l} 
Type & $\varepsilon(\mathrm{kcal} / \mathrm{mol})$ & $\sigma_{0}(\AA)$ & Ref. \\
\hline \hline $\mathrm{CH}_{2}-\mathrm{CH}_{2}$ & 0.091014 & 3.93 & {$[159]$} \\
\hline $\mathrm{CH}_{3}-\mathrm{CH}_{3}$ & 0.212631 & 3.91 & {$[159]$} \\
\hline $\mathrm{S}-\mathrm{S}$ & 0.39743 & 4.25 & {$[46]$} \\
\hline cross-interactions & $\varepsilon_{i j}=\sqrt{\varepsilon_{i i} \varepsilon_{j j}}$ & $\sigma_{0_{i j}}=\left(\sigma_{0_{i i}}+\sigma_{0_{j j}}\right) / 2$ & {$[70]$}
\end{tabular}

Table 4.5: Lennard-Jones parameters for non-bonded thiol-thiol interactions.



Figure 4.2: Non-bonded interactions between different atom species. 


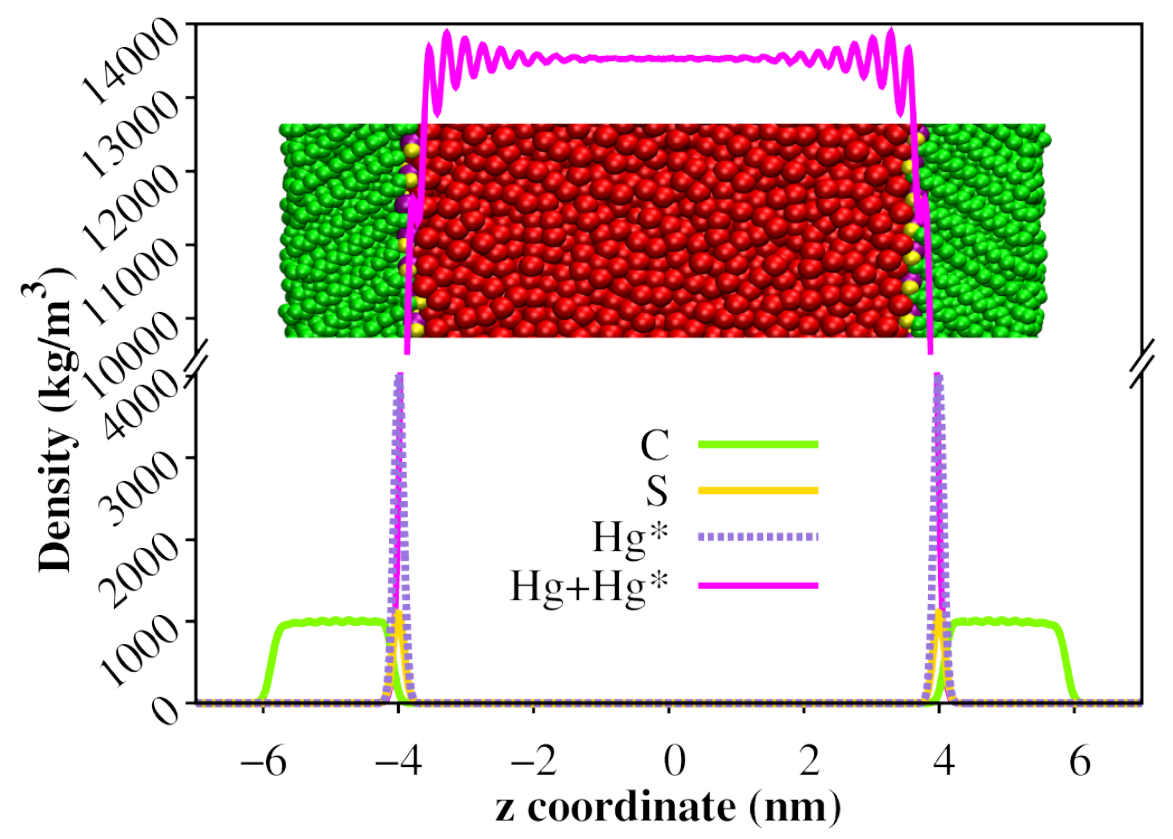

Figure 4.3: Schematic depiction of the simulation box.

\subsubsection{Setup of molecular systems and equilibration: Preliminary insights into the self-assembly of thiols on liquid $\mathrm{Hg}$}

The setup and equilibration of the molecular systems under investigation are highly nontrivial tasks and require a separate attention. This is why I am going to discuss herein these procedures in detail. I use a slab geometry, in which the mercury film is located in the middle of the slab and an equal amount of thiols is placed on each side of the $\mathrm{Hg}$ film. A schematic view of the simulation box is shown in Fig. 4.3. The thickness of the film is about $8 \mathrm{~nm}$, which ensures that the two surfaces of liquid mercury are separated by a sufficient amount of the bulk of liquid mercury. As in Chap. 3, the LAMMPS package [77] is used for my MD simulations. In order to accelerate the simulations I apply the reversible multi-time-scale Molecular Dynamics method [83], where the time steps for the non-bonded and bonded interactions were 2 and 0.5 fs, respectively. The temperature is controlled by the Nosé-Hoover chain thermostat [80] of the length of ten coupled thermostats. After equilibration the sampling for the analysis is done every 2 ps. 


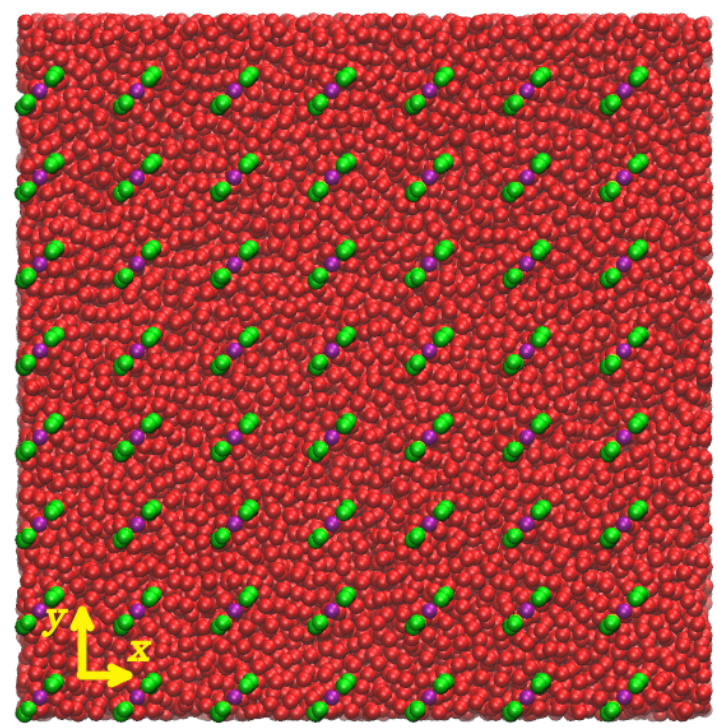

(a)
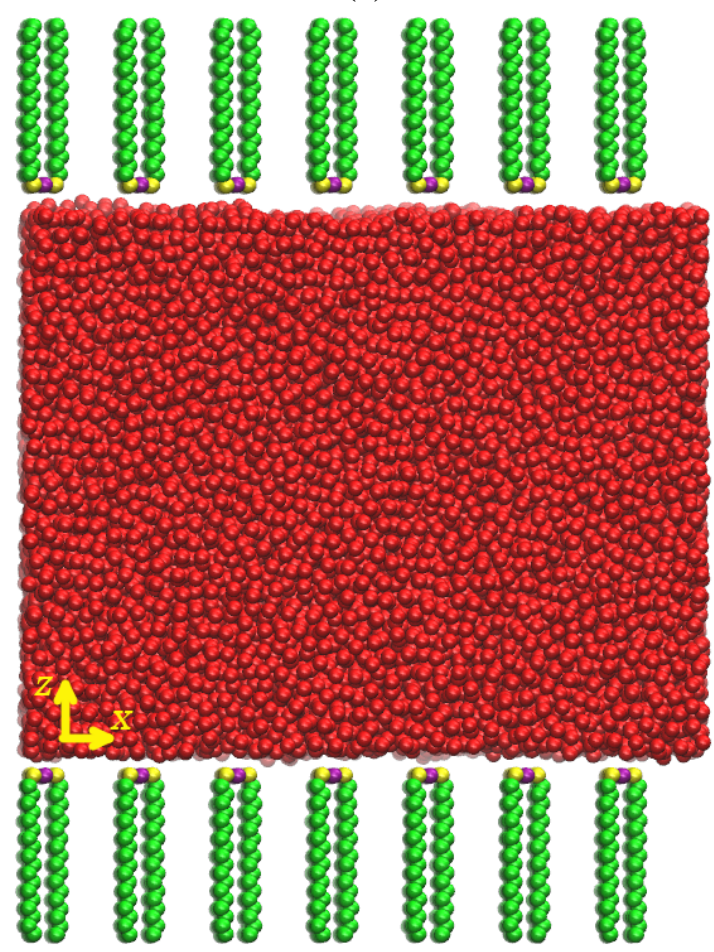

(b)

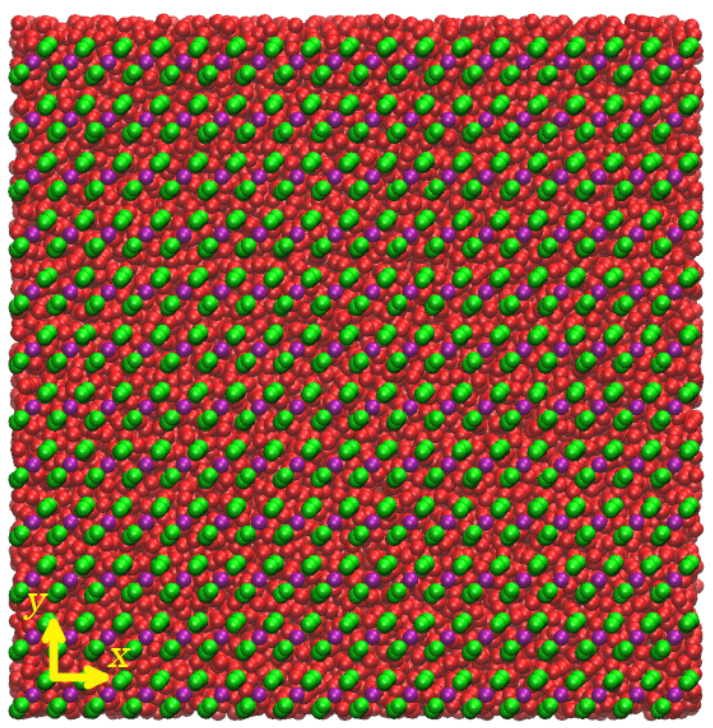

(c)
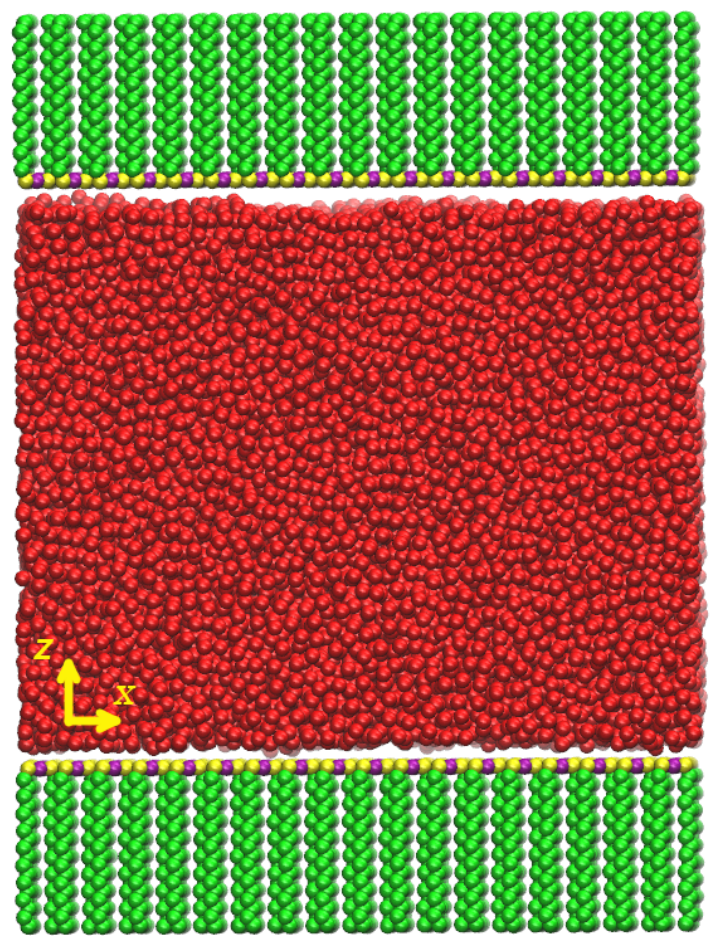

(d)

Figure 4.4: Example of the initial setup of octadecanethiols (SC18) on liquid Hg. Top (a) and side (b) views for $\sigma=1.1156 \mathrm{~nm}^{-2}$. Top (c) and side (d) views for $\sigma=4.3 \mathrm{~nm}^{-2}$, apart from the thiols standing upright this system (c, d) corresponds to the experimental packing in dense thiol SAMs on Hg [64]. The $\mathrm{S}-\mathrm{Hg}-\mathrm{S}$ bonds are rotated by $45^{\circ}$ with respect to the $y$-axis. The shown systems correspond to the system cross-section of $9.936 \times 10.104=100.39 \mathrm{~nm}^{2}$. Color code: bulk $\mathrm{Hg}$ (red); bound $\mathrm{Hg}^{*}$ (purple), sulfur (yellow), alkyl tails (green). 
As the first simple choice, I have distributed the surfactant molecules ( $\mathrm{R}-\mathrm{S}-\mathrm{Hg}-\mathrm{S}$ $\mathrm{R})$ with their alkyl tails standing untilted in all-trans conformations on the sites of a simple square lattice as shown in Fig. 4.4. After such initialization (for low values of $\sigma)$ the alkyl tails fall down and spread across the $\mathrm{Hg}$ surface within the first $0.2-0.5 \mathrm{~ns}$. For a given thiol surface coverage, $\sigma$, the lattice spacings in $x$ - and $y$-directions were chosen such that the molecules were uniformly spaced along the $x$ - and $y$-axes. The thiol surface coverage is defined as the number of thiol molecules per one side of the $\mathrm{Hg}$ film, $N_{t h}$, divided by the total surface cross-section, $A_{c s}$, as follows

$$
\sigma=\frac{N_{t h}}{A_{s c}}
$$

where $N_{t h}=2 N_{m}$ and $N_{m}$ is, consequently, the number of surfactants per one side of the $\mathrm{Hg}$ film. Finally, $A_{c s}=L_{x} L_{y}$, where $L_{x}$ and $L_{y}$ are the lateral extensions of the simulation box in $x$ - and $y$-directions. Prior to placing surfactants onto the $\mathrm{Hg}$ surface the liquid $\mathrm{Hg}$ film was pre-equilibrated for $8 \mathrm{~ns}$. In the following I designate this way of initializing the systems as the $U$-initialization. Periodic boundary conditions are applied in all directions. Sufficient amount of empty space was added above and below the Hg film in order to insure that the molecules on the top and bottom sides of the $\mathrm{Hg}$ film do not influence each other.

As discussed above, experiments indicate a transition between laying-down and standing-up thiols as the surface coverage increases above the full coverage of the laying-down conformations [64]. As the first attempt to investigate this effect, I study the SC18 self-assembly for a wide range of the surface coverage values and total system cross-section, $A_{c s}$, of $9.936 \times 10.104 \approx 100.39 \mathrm{~nm}^{2}$. Tab. A.1 gives a brief overview of equilibration, $t_{e q}$, and sampling, $t_{s}$, times as well as of the final surfactant configurations obtained after the total simulation time, $t_{t o t}=t_{e q}+t_{s}$, was reached. From this table one can see that it is extremely difficult (i.e. takes a lot of computational time) to obtain standing-up thiol conformations for the intermediate range of $\sigma$ values above the full coverage, $\sim 0.8 \mathrm{~nm}^{-2}$, of the laying-down thiols up to $\sigma \approx 1.59 \mathrm{~nm}^{-2}$. In the following I will call this region of $\sigma$ values the oversaturated one. For example, for $\sigma=1.1156 \mathrm{~nm}^{-2}$ no standing molecular conformations were observed after the total simulation time of $238 \mathrm{~ns}$ was reached, although the surface coverage is clearly above the complete monolayer of the laying-down SC18 molecules (see Fig. 4.5a). For 


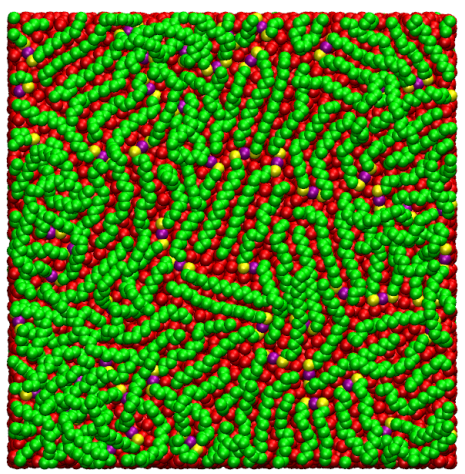

(a) $A_{c s}=100.39 \mathrm{~nm}^{2}, 238 \mathrm{~ns}$.

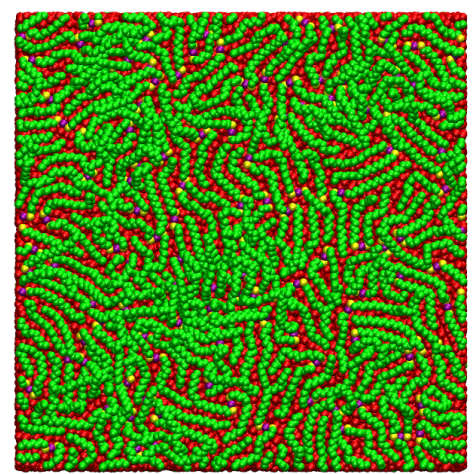

(b) $A_{c s}=225.9 \mathrm{~nm}^{2}, 218 \mathrm{~ns}$.

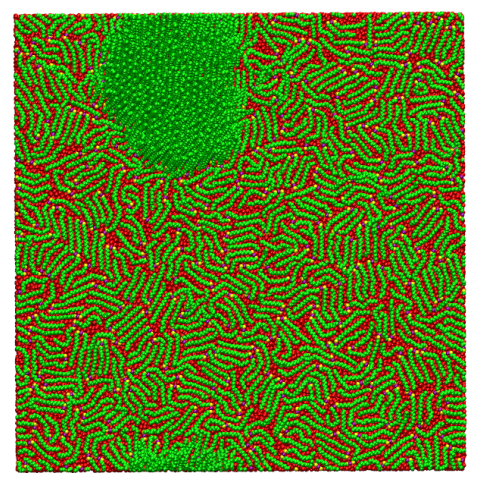

(c) $A_{c s}=903.5 \mathrm{~nm}^{2}, 128 \mathrm{~ns}$.

Figure 4.5: Example of the finite-size effects on the structure of the SC18 SAM at indicated times. Snapshots from MD simulations for $\sigma \approx 1.1156 \mathrm{~nm}^{-2}$ and different system cross-sections $A_{c s}$. Color code same as in Fig. 4.4 .

$\sigma=1.27498 \mathrm{~nm}^{-2}$ an island of standing thiols was formed only on one side of the $\mathrm{Hg}$ film after $279.5 \mathrm{~ns}$. For $\sigma=1.43436 \mathrm{~nm}^{-2} \mathrm{I}$ have carried out two simulation runs each of 278.2 and $339.95 \mathrm{~ns}$ long, respectively. In the first run I observed the formation of crystalline islands of thiols from both sides of the $\mathrm{Hg}$ film after $133.2 \mathrm{~ns}$. In the second run I was not able to obtain the islands on both sides of the film even after $339.95 \mathrm{~ns}$. For $\sigma=1.59373 \mathrm{~nm}^{-2}$ I have performed also two separate simulation runs and this time in both cases I was able to obtain crystalline thiol islands on both sides of the $\mathrm{Hg}$ film. Although the times, after which the islands were formed on the both sides, were substantially different in this two runs and comprised 225.936 and $120 \mathrm{~ns}$, respectively. All this indicates that the formation of crystalline islands in the oversaturated region is a protracted spontaneous process that heavily depends on the lateral system sizes. Upwards from this value of $\sigma$ I was able to resolve crystalline standing phases of octadecanethiols with the equilibration times generally lesser than the ones in the oversaturated region.

At this point I have to clarify what is meant by $t_{e q}$ for various ranges of $\sigma$. For $\sigma$ values lower than that of the full coverage of laying down thiols, $t_{e q}$ is the time, at which both, the system total energy and surface tension, level off and start to fluctuate around their mean values. Thus, I consider that such a system has reached the thermodynamic equilibrium for the simulated temperature. If a molecular system is in the oversaturated region of $\sigma$ values and has not developed crystalline thiol phases from both sides of the 
$\mathrm{Hg}$ film during the whole simulation time, $t_{t o t}$, I consider $t_{e q}$ to have the same meaning as above. Although, in this case, such an "equilibrium state" is not the true thermodynamic equilibrium state at the given temperature and surface coverage, and only represents a steady state of an oversaturated layer of laying-down molecules, which is simply a result of finite-size effects. Now, if the system in the oversaturated region has developed a crystalline island only on one side of the film, I consider such system to be not equilibrated and will discard such systems for the following analysis. Finally, if a system has developed a crystalline island on each side of the $\mathrm{Hg}$ film, it will be equilibrated, when again both, its surface tension and total energy, start to fluctuate around their mean values. I should also note that for various system sizes the size of the oversaturated region varies (compare SC18 structures for $\sigma \approx 1.1156 \mathrm{~nm}^{-2}$ and different $A_{c s}$ in Fig. 4.5 ). In fact, we will see that for larger system cross-sections the oversaturated region shrinks.

Tab. A.2 gives an overview of studied dodecanethiol (SC12) systems for the crosssection of $9.936 \times 10.104=100.39 \mathrm{~nm}^{2}$. Here the full coverage of laying-down thiols is attained at about $\sigma=1.1156 \mathrm{~nm}^{-2}$. Starting from this value of $\sigma$ and up to $\sim 2.07 \mathrm{~nm}^{-2}$ the systems stayed in oversaturated states of laying-down thiols with some strongly disordered patches of semi standing molecules during the total simulation time. Giving such large finite-size effects, this systems will be omitted in the further treatment.

To reduce the discussed above finite-size effects the octadecanethiol (SC18) systems of larger cross-section, $A_{c s}$, of $14.904 \times 15.156 \approx 225.9 \mathrm{~nm}^{2}$ have been set up (see Tab. A.3). The width of the $\mathrm{Hg}$ film was left approximately unaltered $(\sim 8 \mathrm{~nm}$ ). For this system cross-section the oversaturated region is slightly shorter (compared to smaller systems) and goes from the full coverage of laying-down thiols up to $\sigma \approx$ $1.1156 \mathrm{~nm}^{-2}$. The respective oversaturated SC18 system at $\sigma=1.1156 \mathrm{~nm}^{-2}$ is still not able to develop a crystalline phase of the standing-up thiols (see Fig. 4.5b). In order to reduce the finite-size effects even further, I have set up two SC18 systems for $A_{c s}=29.808 \times 30.312 \approx 903.5 \mathrm{~nm}^{2}$ and thiol surface coverages of 1.1156 and $1.43879 \mathrm{~nm}^{-2}$, respectively. Only at such huge system cross-section (as for an atomistic computer simulation) I was able for the first time to resolve a SC18 crystalline island for $\sigma=1.1156 \mathrm{~nm}^{-2}$ as is shown in Fig. $4.5 \mathrm{c}$. Unfortunately, at the same time, I have run here into several problems simultaneously.

On one hand, the equilibration times remain very large. For $\sigma=1.43879 \mathrm{~nm}^{-2}$ the islands of standing-up thiols on both sides of the $\mathrm{Hg}$ film are observed only after 


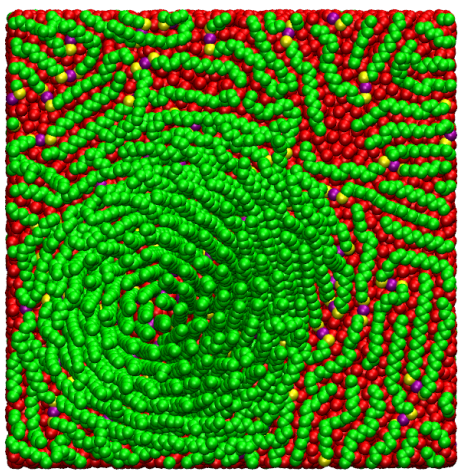

(a) Metastable spiral island.

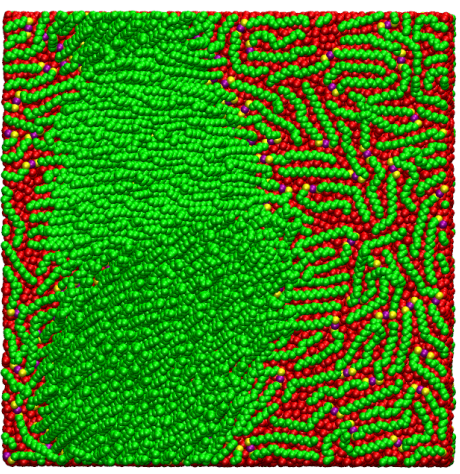

(b) Grain boundary.

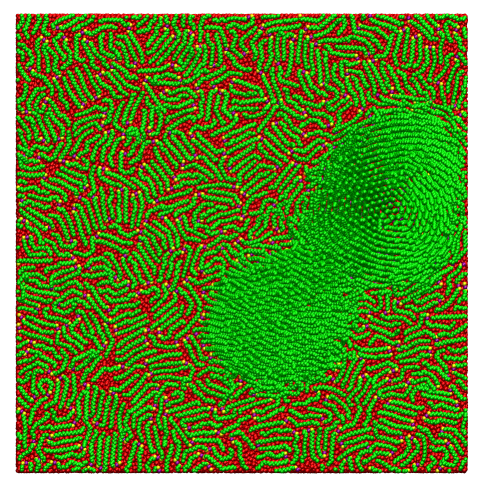

(c) Grain boundary.

Figure 4.6: Possible defects of SAMs on liquid $\mathrm{Hg}$. (a) $A_{c s}=100.39 \mathrm{~nm}^{2}, \sigma=$ $2.07185 \mathrm{~nm}^{-2}$ and $t_{t o t}=294.92 \mathrm{~ns}$; (b) $A_{c s}=225.9 \mathrm{~nm}^{2}, \sigma=2.07185 \mathrm{~nm}^{-2}$ and $t_{\text {tot }}=203.75 \mathrm{~ns}$; (c) $A_{c s}=903.5 \mathrm{~nm}^{2}, \sigma=1.43879 \mathrm{~nm}^{-2}$ and $t_{\text {tot }}=123.85 \mathrm{~ns}$; Color code same as in Fig. 4.4 .

$t_{e q}=100 \mathrm{~ns}$. And for $\sigma=1.1156 \mathrm{~nm}^{-2}$ and $t_{t o t}=128.45 \mathrm{~ns}$ the system has developed a crystalline thiol island only on one side of the $\mathrm{Hg}$ film. On the other hand, the Uinitialized systems develop various multiple defects in SAMs (see Fig. 4.6), relaxation times of which are basically unreachable on the typical time scales of my simulations.

In order to overcome these obstacles I have adopted an advanced initialization procedure for the systems with the largest simulated cross-section, $A_{c s}$, of $903.5 \mathrm{~nm}^{2}$. For the systems with the values of thiol surface coverage $\sigma \geq 1.1156 \mathrm{~nm}^{-2}$ crystalline islands of standing-up thiols with a given number of thiol molecules were pre-initialized on the surface of liquid mercury. Such islands were surrounded by a "sea" of pre-equilibrated laying-down octadecanethiols as illustrated in Fig. 4.7). To achieve a desired surface coverage a specific number of molecules of the number of the laying-down ones are removed from the system and on their place the island of standing-up thiols is inserted. Afterwards, such molecular configuration is reflected onto the opposite side of the $\mathrm{Hg}$ film. In the following I will call such initialization procedure the P-initialization. The number of thiols in the crystalline islands was approximately estimated where possible from the previous simulations of the systems, which had the same cross-section and surface coverage values and were initialized with the help of the U-initialization procedure. For the other systems the percentage of standing molecules was determined from the simulations of the systems with smaller cross-sections and similar values of the 

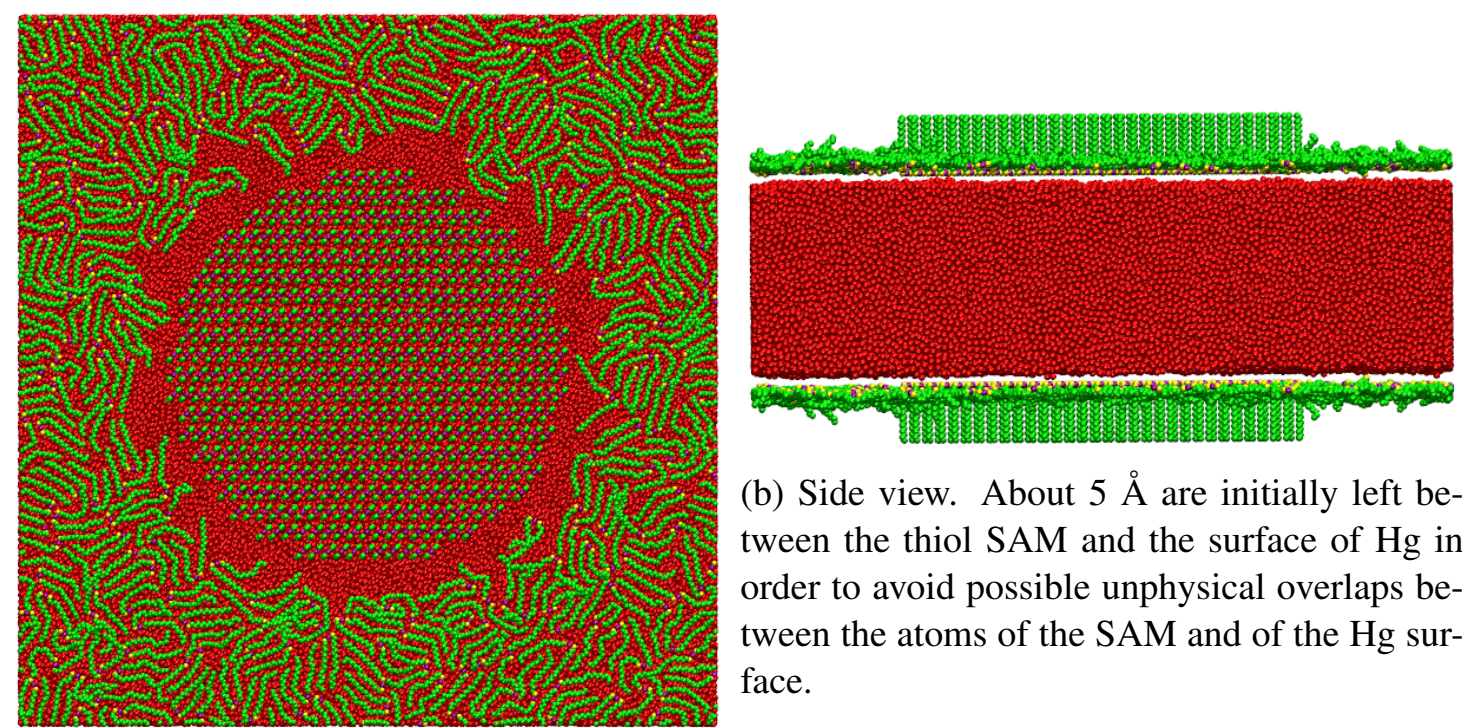

(b) Side view. About $5 \AA$ are initially left between the thiol SAM and the surface of $\mathrm{Hg}$ in order to avoid possible unphysical overlaps between the atoms of the SAM and of the Hg surface.

(a) Top view.

Figure 4.7: Example of the initial setup of the SC18 monolayer for $\sigma=1.61144 \mathrm{~nm}^{-2}$ with a crystalline island of the standing-up thiols. The system cross-section is $903.5 \mathrm{~nm}^{2}$. The arrangement of thiols in the island and the color code are identical to Figs. $4.4 \mathrm{c}$ and $4.4 \mathrm{~d}$.

thiol surface coverage. I have presumed an experimentally proposed rectangular unitcell [64] with two thiols per cell and the lengths of the base vectors of 5.52 and $8.42 \AA$ for the molecular arrangement in the islands. The alkyl tails in the thiol islands were set in the all-trans conformations and left untilted in order to bias the system lesser and to allow it to relax into the state with an equilibrium tilt angle itself. The initial structure of in such a way obtained thiol islands is identical to the one shown in Figs. 4.4c and 4.4d, In the system with $\sigma=2.10948 \mathrm{~nm}^{-2}$ a stripe of standing molecules spanning through the periodic boundary was initialized using the procedure described above. It takes at most $20 \mathrm{~ns}$ to equilibrate the systems that were set up by the P-initialization procedure. The rest of the octadecanethiol systems at lower surface coverage $\left(\sigma<1.1156 \mathrm{~nm}^{-2}\right)$ were set up via the U-initialization. All octadecanethiol systems with the cross-section of $A_{c s}=29.808 \times 30.312 \approx 903.5 \mathrm{~nm}^{2}$ are listed in Tab. A.4.

Since it was impossible to resolve dodecane (SC12) crystalline islands for the systems of smaller cross-section, I have pre-set up SC12 systems with the crystalline island by simply trimming (T-initialization) octadecanethiols in the respective equilibrated 
SC18 systems $\left(\sigma=1.43879\right.$ and $\left.1.61144 \mathrm{~nm}^{-2}\right)$ to the needed length. In such a way prepared SC12 systems needed considerably longer equilibration times (see Tab. A.5) than the respective SC18 systems initialized by the P-initialization. This is explained by the fact that thus obtained crystalline islands of dodecanethiols were too large and had to partially melt away. This is why I have pre-set up the SC12 system for $\sigma=2.00545$ by applying the P-initialization. The respective number of the dodecanethiols in the crystalline island has been simply taken to be approximately 50\% larger than the one in the equilibrated SC12 system with $\sigma=1.61144 \mathrm{~nm}^{-2}$. This allowed to equilibrate the system in $22 \mathrm{~ns}$. Low-coverage SC12 systems were initialized by using the U-initialization procedure. In general, all high-coverage SC12 systems with $A_{c s}=903.5 \mathrm{~nm}^{2}$ required longer sampling times because of strong shape oscillations of the crystalline islands. Details on the dodecanethiol systems for $A_{c s}=903.5 \mathrm{~nm}^{2}$ used in my simulations are presented in Tab. A.5. In the following analysis I use mostly SC18 and SC12 surfactant systems with the largest simulated cross-section area of $903.5 \mathrm{~nm}^{2}$. Finally, the largest simulated system ( $\mathrm{SC} 18$ surfactants at $\sigma=2.10948$ and $A_{c s}=903.5 \mathrm{~nm}^{2}$ ) contained in total 360004 particles.

\subsubsection{Computational efficiency of LAMMPS}

Fig. 4.8 shows the LAMMPS speedup as a function of a number of CPUs for the systems with the cross-section, $A_{c s}$, of $903.5 \mathrm{~nm}^{2}$ and various number of surfactants per side of the $\mathrm{Hg}$ film, $N_{m}$. The dynamical reassignment of the particles to the computational cores is used in order to optimize the load balance. This is why the respective plots of the speedup are not ideally monotonous. The data was obtained on the system Cray XC40 with the CPU architecture Intel Xeon Haswell (12 cores with 2 CPUs each, CPU model E5-2680v3, CPU clock 2.5 GHz). The scaling behavior of LAMMPS for all values of $N_{m}$ is approximately the same, as is seen from Fig. 4.8. Additionally, it is clear from the figure that the LAMMPS scalability starts to substantially depart from the ideal one at \#CPUs $=196$. This is why I have used 196 CPUs for the simulations of all the systems with $A_{c s}=903.5 \mathrm{~nm}^{2}$. A typical simulation of about $6 \mathrm{~ns}$ for such systems sizes on 200 cores takes 12 hours. For the values of $A_{c s}$ equal to 100.39 and $225.9 \mathrm{~nm}^{2}$ a similar scalability analysis has been done, based on which it has been decided to carry out the simulations on 60 and 100 \#CPUs, respectively. 


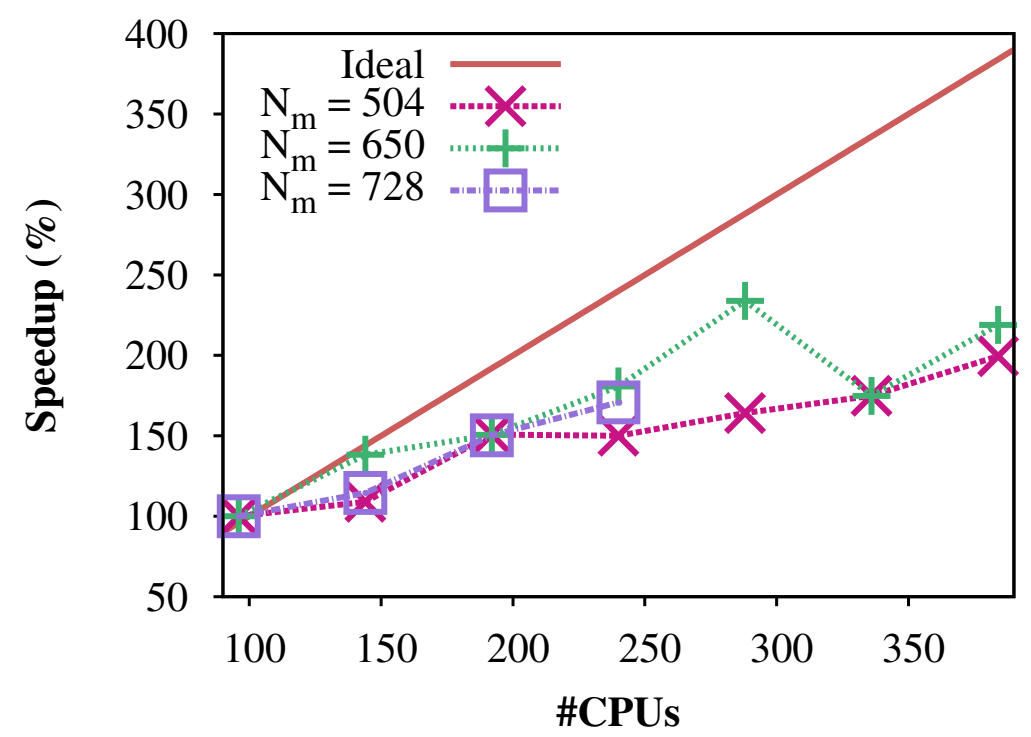

Figure 4.8: LAMMPS speedup normalized to 96 cores as a function of a number of CPUs. The data is presented for the systems with the cross-section of $903.5 \mathrm{~nm}^{2}$ and various number of surfactants per side of the $\mathrm{Hg}$ film, $N_{m}$. Hardware: Intel Xeon Haswell (12 cores with 2 CPUs each, CPU model E5-2680v3, CPU clock $2.5 \mathrm{GHz}$ ); system: Cray XC40.

\subsection{Coexistence of various alkylthiol phases on mercury}

\subsubsection{Laying-down vs. standing-up phases}

Let us now take a first glance at the phase behavior of thiols on liquid mercury depending on their surface coverage, $\sigma$. I start first by looking at the octadecanethiol (SC18) conformations, which are shown in Fig. 4.9. One sees that at low surface coverage $\left(\sigma<0.83 \mathrm{~nm}^{-2}\right)$ thiols prefer to aggregate into clusters of laying-down molecules. There are some correlations in the orientation of molecular axes (up to 5-6 thiols) of the laying-down molecules, but, generally, the structure of these laying-down agglomerates is random. The agglomerates of laying-down octadecanethiols will grow until the full coverage of the laying-down conformations at $\sigma \approx 0.8 \mathrm{~nm}^{-2}$ (see Tab. A.1) is reached. Generally, as $\sigma$ increases above the full coverage, some octadecanethiol molecules stand up with their alkyl tails tilted at a sharp angle to the surface normal and begin to form crystalline clusters. Nevertheless, due to finite size effects oversaturated 


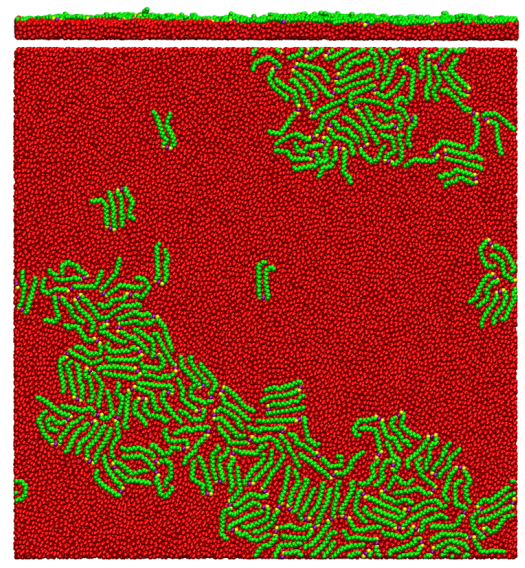

(a) $\sigma=0.26562 \mathrm{~nm}^{-2}$

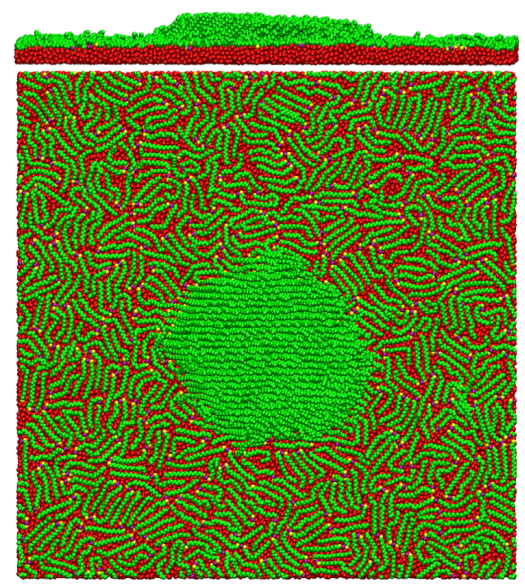

(c) $\sigma=1.1156 \mathrm{~nm}^{-2}$

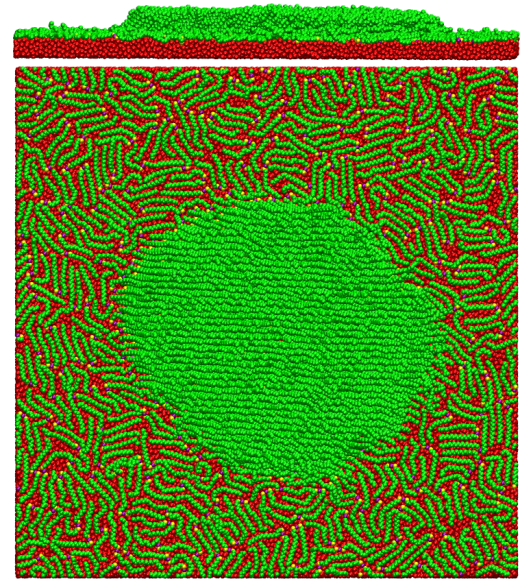

(e) $\sigma=1.61144 \mathrm{~nm}^{-2}$

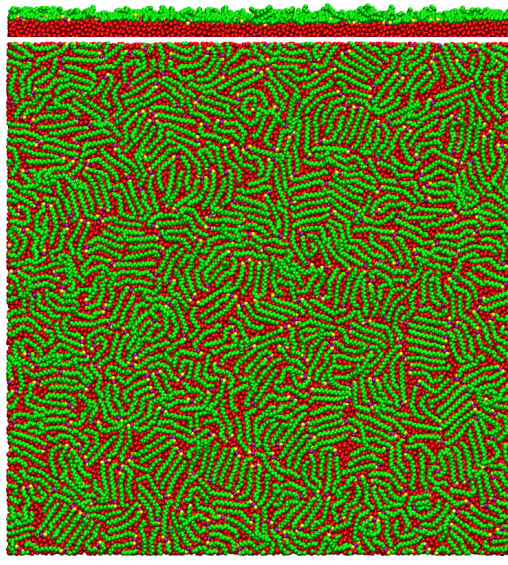

(b) $\sigma=0.83671 \mathrm{~nm}^{-2}$

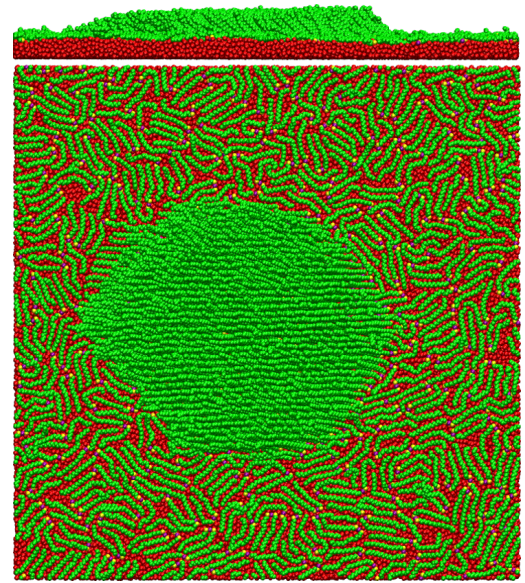

(d) $\sigma=1.43879 \mathrm{~nm}^{-2}$

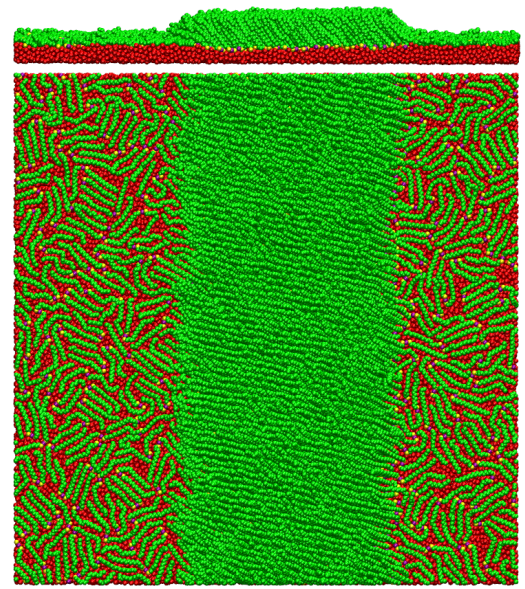

(f) $\sigma=2.10948 \mathrm{~nm}^{-2}$

Figure 4.9: Various phases of octadecanethiols on liquid $\mathrm{Hg}$ as a function of surface coverage, $\sigma . A_{c s}=29.808 \times 30.312 \approx 903.5 \mathrm{~nm}^{2}, \mathrm{t}_{\text {tot }}=107 \mathrm{~ns}$. 


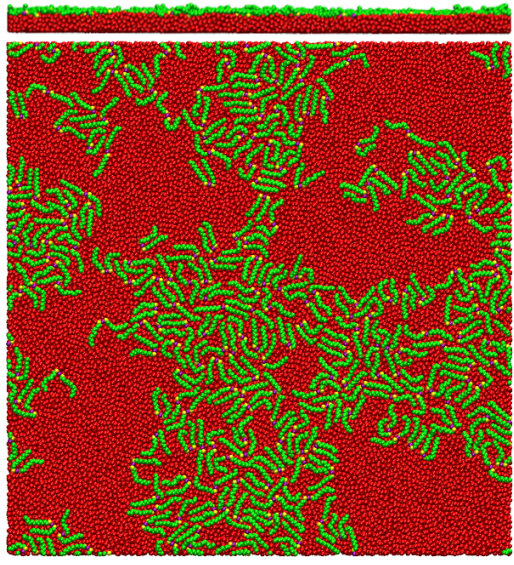

(a) $\sigma=0.53124 \mathrm{~nm}^{-2}$

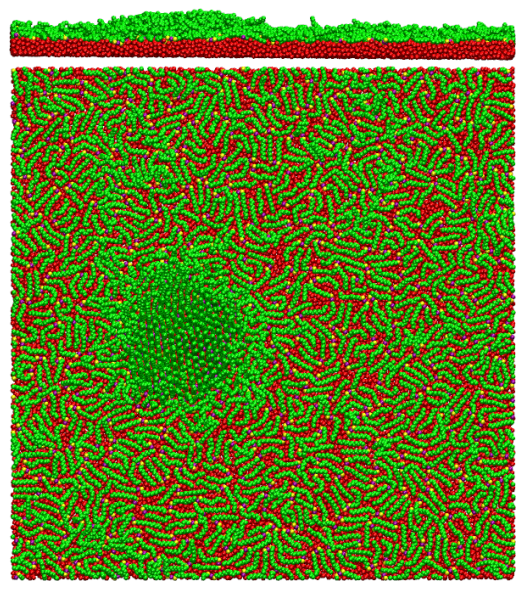

(c) $\sigma=1.43849 \mathrm{~nm}^{-2}$

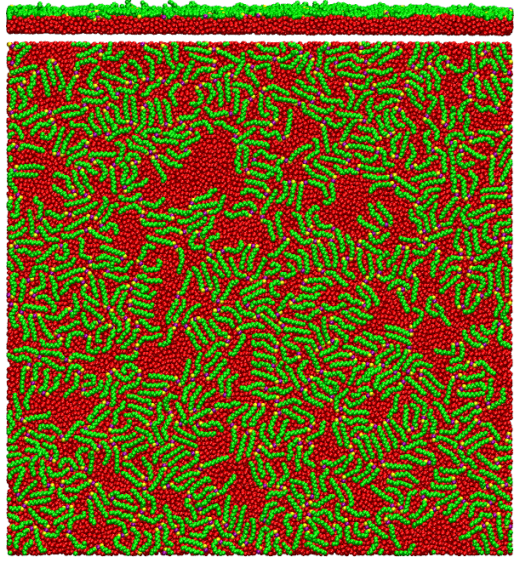

(b) $\sigma=0.83671 \mathrm{~nm}^{-2}$

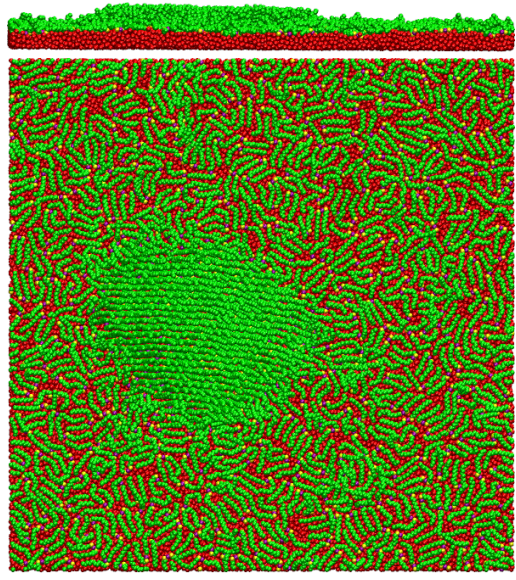

(d) $\sigma=1.61144 \mathrm{~nm}^{-2}$

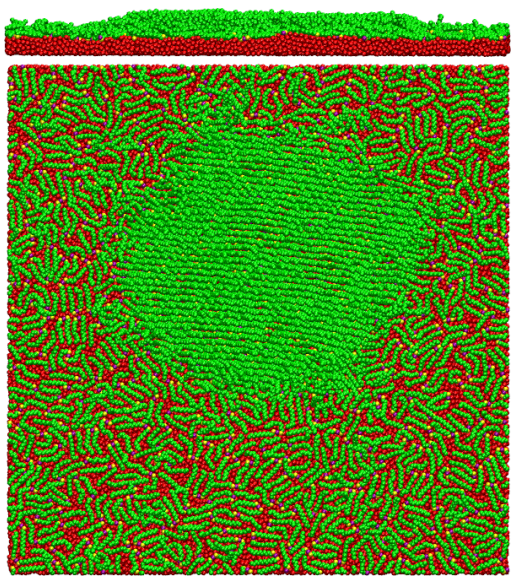

(e) $\sigma=2.00545 \mathrm{~nm}^{-2}$

Figure 4.10: Various phases of dodecanethiols on liquid $\mathrm{Hg}$ as a function of surface coverage, $\sigma$. $A_{c s}=29.808 \times 30.312 \approx 903.5 \mathrm{~nm}^{2}$. 


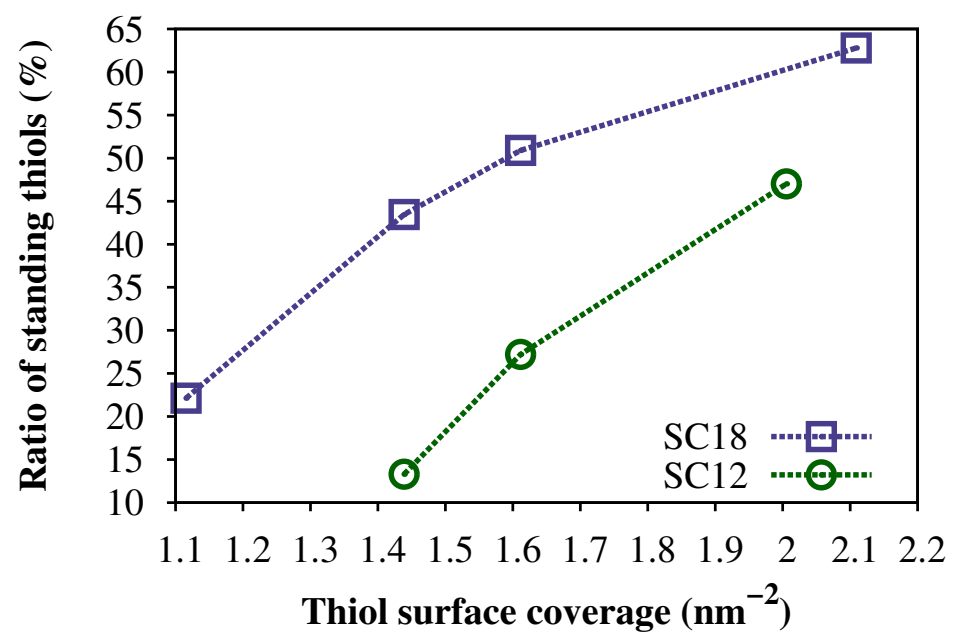

Figure 4.11: Ratio of standing alkylthiols for various values of thiol surface coverage.

monolayers of laying-down octadecanethiols exist until the number of surfactants will suffice to build up a bulky standing island. Fig. $4.9 \mathrm{~b}$ depicts one of such oversaturated states for $\sigma=0.83671 \mathrm{~nm}^{-2}$. We will see later that such slightly oversaturated layer results in the value of the surface tension, $\gamma$ below the coexistence plateau in the $\gamma-\sigma$ isotherm. Figs. $4.9 \mathrm{c}-\mathrm{f}$ demonstrate the coexistence between the monolayers of layingdown and standing-up SC18 thiols for a wide range of values of the surface coverage. As is clearly seen from the snapshots the size and form of the islands of standing-up thiols depend on $\sigma$. It is also interesting to note that the molecules are not tilted uniformly across the whole islands, but rather gradually increase their tilt starting at a given side of the island, where the thiols from the first row have their alkyl tails laying flat on the $\mathrm{Hg}$ surface. In turn, the tails of the second molecular row lay on top of the molecules from the previous row and so forth. For large enough islands the effect of the nonuniform tilting vanishes for most of the molecules (see Fig. 4.9e). The dodecanethiol (SC12) phase behavior with respect to the increase of $\sigma$ is similar to the one of the SC18 systems (Fig. 4.10). Here the full coverage of the monolayer of the laying-down dodecanethiols corresponds to $\sigma \approx 1.1156 \mathrm{~nm}^{-2}$ (see Tab. A.2). At higher dodecanethiol coverages I observe stable regions of coexistence between laying-down and standing-up dodecanethiols as well (Figs. 4.10-e). Unlike from the SC18 islands, the SC12 ones have very blurred boundaries (compare e.g. Figs 4.10 , d to Figs 4.9 d,e). One also notices 
from the snapshots that on such boundaries the concentration of liquid-like thiols is higher than in the monolayer of laying-down dodecanethiols. This finding correlates with the previous observation that the shape of SC12 islands was strongly fluctuating during the simulations, thus, causing longer sampling times. Finally, Fig. 4.11 gives a comparison of the amount of standing thiols in the octadecanethiol and dodecanethiol crystalline islands. One sees that for lower values of $\sigma\left(1.43849 \mathrm{~nm}^{-2}\right)$, the number of thiols in the SC18 island is about 30\% larger than in the SC12 ones, but, as $\sigma$ goes above $2 \mathrm{~nm}^{-2}$, this difference drops down to about $20 \%$.

\subsubsection{Density profiles}

A more quantitative description of the phase transformations in the alkylthiol SAMs on the surface of liquid mercury offers the analysis of the respective density profiles. The processes of filling in the 1st monolayer of laying-down octadecanethiols as well as of the formation of the standing crystalline thiol islands are clearly mirrored in the density profiles perpendicular to the $\mathrm{Hg}$ film surface (Fig. 4.12a for SC18 systems). One sees that in the low-coverage regime $\left(\sigma \leq 0.8367 \mathrm{~nm}^{-2}\right)$ the density profiles for the SC18 systems are characterized by single sulfur and alkyl peaks, that are spatially very close to each other. The alkyl peak grows in the amplitude up to the point when the full coverage of the monolayer of laying-down octadecanethiols is reached, after attaining which the 1 st peak varies only slightly and the density profile starts to develop a shoulder into the empty space above the $\mathrm{Hg}$ film. At the same time the sulfur peak starts to depart more in the opposite direction as $\sigma$ grows. This is explained by the observation that the sulfur peak consists of two contributions, namely, from the sulfurs in the laying-down and standing-up thiol conformations, respectively. And the sulfurs in the monolayer of the standing molecules are tucked deeper into the bulk of mercury, as is also noticeable in the side view of the SC18 system for $\sigma=2.10948 \mathrm{~nm}^{-2}$ (Fig. 4.9f). As the number of standing octadecanethiols grows the contribution from the sulfurs in the standing-up islands increases and the peak's maximum shifts towards the mercury bulk. Similar effect is also responsible for the slight changes in the height of the 1st alkyl peak in the systems with crystalline islands. The density profiles for the dodecanethiols feature behavior similar to the octadecanethiols discussed above, with the only slight difference that the alkyl shoulders (due to the standing-up molecules) have regions (next to the 1st 
peak) of somewhat increased intensities (compared to the rest of the profile). Such behavior of the SC12 density profiles is contributed to the blurred liquid-like boundary of the crystalline islands in the SC12 systems. The density profiles for the pure crystalline phases will be presented in the next chapter.



(a)

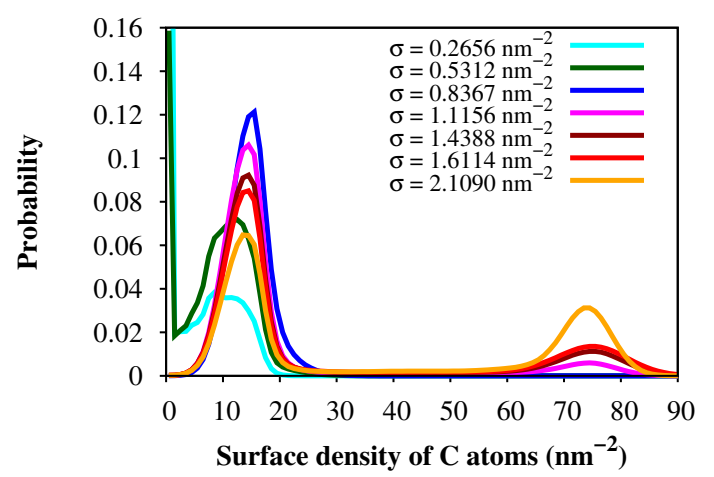

(c)

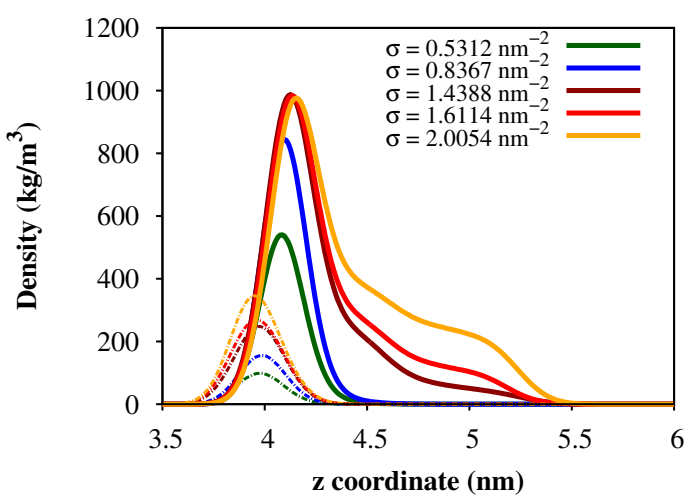

(b)

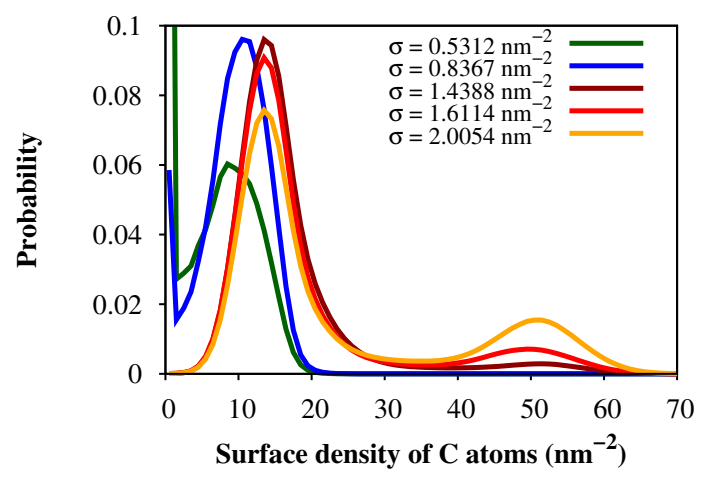

(d)

Figure 4.12: Density profiles of octadecanethiol (a) and dodecanethiol (b) surfactants on the surface of liquid $\mathrm{Hg}$ for various values of molecular surface coverage, $\sigma$. Solid and dash-dot lines stand for the alkyl tails and sulfur density profiles, respectively. Probability distributions of finding specific amount of carbon atoms in a surface element with the cross-section of $1 \times 1 \mathrm{~nm}^{2}$ for various surface coverages, $\sigma$, of octadecanethiol (c) and dodecanethiol (d) self-assembled monolayers on the surface of liquid mercury. 


\subsubsection{Surface density distributions}

Another alternative way of characterizing the coexistence of various thiol phases on liquid mercury is by calculating the probabilities of finding a particular number of alkyl groups (or simply carbon atoms) in a surface element with a cross-section of approximately $1 \times 1 \mathrm{~nm}^{2}$. This surface element should not be to large, otherwise one would wipe away all local surface density fluctuations. On the other hand it should not be unphysically small. An extreme limiting cross-section for such sample surface element would be $\pi \sigma_{\mathrm{CH}_{2}}^{2} \approx 0.5 \mathrm{~nm}^{2}$, where $\sigma_{\mathrm{CH}_{2}}=3.93 \AA$ is the radius of the Lennard-Jones hard-core shell of the alkyl groups. The respective probability distributions for the octadecanethiol and dodecanethiol systems are demonstrated in Figs. 4.12c and 4.12d, We see that in both cases for the low-coverage regime these distributions are characterized by two peaks at 0 and 10 carbons $/ \mathrm{nm}^{2}$, which is an indicator of the coexistence of the monolayer of laying-down molecules with a bare surface of liquid mercury. For the molecular coverages close to the full filling of a monolayer of laying-down molecules one obtains only one single peak, as is nicely seen for octadecanethiols at $\sigma=0.8367 \mathrm{~nm}^{-2}$ (Fig. 4.12c). Since at this value of $\sigma$ the corresponding dodecanethiol system is below the full coverage (see Tab. A.2), two peaks at 0 and $\sim 10$ carbons are still observed for this system. For the surfactant systems with crystalline thiol islands the peak at 0 vanishes and, instead, the second peak at higher carbon numbers arises. In the case of $\sigma=1.6114 \mathrm{~nm}^{-2}$, this peak attains its maximum at around 75 and 49.5 carbons for SC18 and SC12 thiol systems, respectively. This yields the thiol (sulfur) surface density of approximately 4.17 and 4.13 thiols per $1 \mathrm{~nm}^{2}$ in the dense islands of octadecanethiols and dodecanethiols, respectively. Similarly, the surface coverage of the octadecanethiols in the laying-down conformations at the coexistence with the islands of the standing molecules is estimated to be about $0.806 \mathrm{~nm}^{-2}$.

\subsubsection{Surface tension-surface coverage $(\gamma-\sigma)$ isotherms}

From the thermodynamic point of view two coexisting phases of matter at the same temperature coexist at equal values of pressure and chemical potential. Meaning that for the two observed types of phase coexistence, namely, of laying-down thiols with bare $\mathrm{Hg}$ surface and of laying-down with standing-up thiols, one should observe respective plateaus in the pressure versus thiol surface coverage values, $\sigma$, for each coexis- 


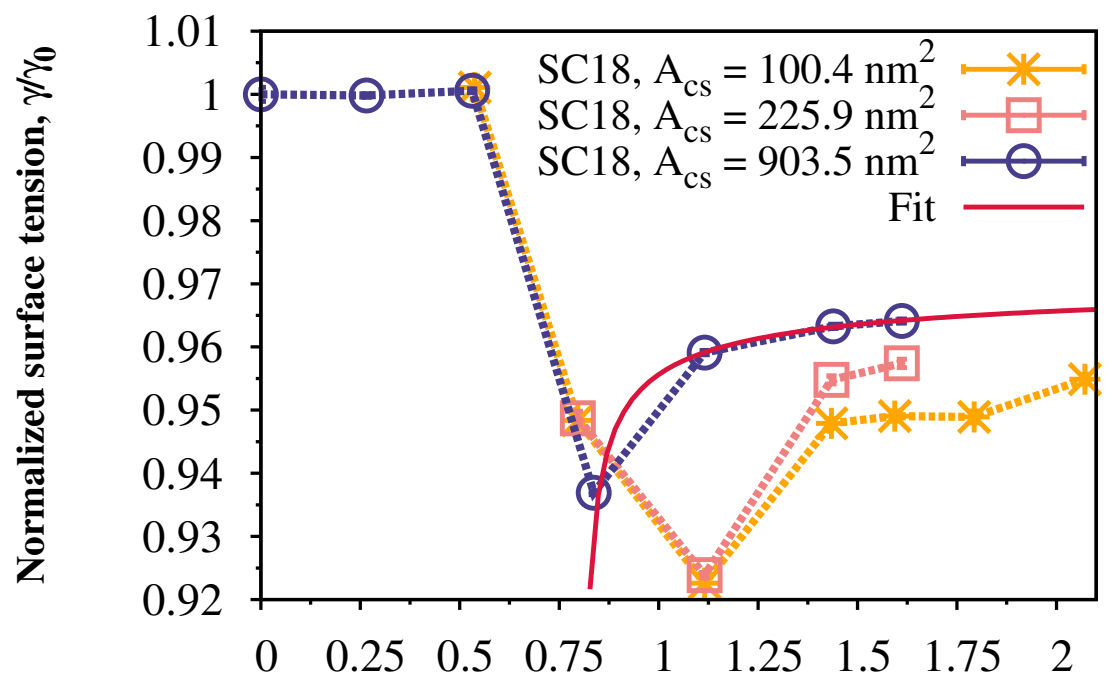

Surface coverage, $\sigma\left(\mathrm{nm}^{-2}\right)$

Figure 4.13: Normalized surface tension, $\gamma / \gamma_{0}$, for various values of octadecanethiol surface coverage, $\sigma$, and system cross-section, $A_{c s} . \gamma_{0}=286.4 \mathrm{mN} / \mathrm{m}$ is the surface tension of the bare surface of liquid mercury of the ODI model for $A_{c s}=903.5 \mathrm{~nm}^{2}$ and $T=293 \mathrm{~K}$. The red solid line is the fit by Eq. 4.18 of $\gamma(\sigma)$ obtained from the MD simulations for $A_{c s}=903.5 \mathrm{~nm}^{2}$. The dashed lines are the guides for the eyes.

tence region. This requirement intuitively translates into the condition of the respective plateaus in the surface tension, $\gamma$, versus $\sigma$ measurements at constant temperature, i.e. in the $\gamma-\sigma$ isotherm. This is also straight forward to see giving the Kirkwood-Buff formula for the surface tension (Eq. 3.17), which I am going to use in order to explore if it is possible to resolve the $\gamma-\sigma$ isotherms for the thiol self-assembled monolayers within the atomistic/UA approach employed in the current work. It appears that this ability is heavily influenced by the finite-size effects, which I am going to discuss in detail in the following. Fig. 4.13 shows the $\gamma-\sigma$ isotherms for octadecanethiol systems and various system cross-sections, $A_{c s}$. In this figure one can clearly distinguish two plateau regions, one for $\sigma$ smaller than the one of the full coverage of completely filled monolayer of laying-down octadecanethiols at $\sigma \approx 0.8 \mathrm{~nm}^{-2}$, and another plateau region at higher values of $\sigma$, at which the coexistence of laying-down and standingup phases is observed (see Tabs. A.1. A.3 and A.4 as well as Fig. 4.9. The values of surface tension are computed only for the systems, which do not have crystalline thiol 
subphases spanning across the boundaries, because in this case the calculation of $\gamma$ via the virial (i.e. pressure in the Kirkwood-Buff relation) is impossible and the meaning of the surface tension itself changes. In Fig. 4.13 one sees also that the very last value of $\gamma$ at $\sigma=2.07185 \mathrm{~nm}^{-2}$ for $A_{c s}=100.39 \mathrm{~nm}^{2}$ overshoots the general level of the 2 nd plateau. As we recall, at these values of $\sigma$ and $A_{c s}$ a metastable spiral island of standing thiols on one side of the $\mathrm{Hg}$ film was observed (Fig. 4.6a). Apparently to stabilize such structure the system requires higher lateral pressure (in the modulo sense) compared to the systems on the plateau. The two plateau regions in Fig. 4.13 are separated by the oversaturated region, where the surface tension drops notably below the second plateau. As discussed above (see Sec. 4.1.2) this oversaturated region strongly depends on the size of the system cross-section, $A_{c s}$, and corresponds to the oversaturated layers of the laying-down octadecanethiols. One can also clearly see this in my $\gamma-\sigma$ isotherms. The oversaturated region of $\sigma$ values shrinks closer to the left, i.e. closer to the value at the full coverage of the laying-down monolayer. Let us take a closer look at the systems for $\sigma=1.1156 \mathrm{~nm}^{-2}$, which represents a vivid example of the finite-size effects in the oversaturated region. At this surface coverage octadecanethiols are not able to form a crystalline island and reside in an oversaturated layer of laying-down molecules for the $A_{c s}$ values of 100.39 and $225.9 \mathrm{~nm}^{2}$ (see Figs. $4.5 \mathrm{a}$ and $4.5 \mathrm{~b}$ as well as Tabs. A.1 and A.3. This results in the values of $\gamma$ considerably lower compared to the respective plateaus (Fig. 4.13). But for $A_{c s}=903.5 \mathrm{~nm}^{-2}$ the number of surfactants at $\sigma=1.1156 \mathrm{~nm}^{-2}$ suffices to form crystalline islands of the standing-up molecules, and, consequently, its $\gamma$ value falls onto the second plateau together with the other surface tension values at higher coverages, which correspond to larger sizes of the thiol crystalline islands. I ascribe small deviations in the surface tension from the "horizontal" level in the plateau in the $\sigma$ region from 1.1156 to $1.6114 \mathrm{~nm}^{-2}$ to the contributions due to the line tension, $\tau$, on the boundary between laying-down and standing-up phases. Typical line tension values for the alkyl-based systems range from $10^{-12}$ to $10^{-9} \mathrm{~J} / \mathrm{m}$ [161, 162]. Thus for the island of standing-up thiols with the diameter, $d$, of approximately $10 \mathrm{~nm}$ at $\sigma=1.1156 \mathrm{~nm}^{-2}\left(A_{c s}=903.5 \mathrm{~nm}^{2}\right)$, the maximal line tension contribution to the surface energy is $\pi d \tau \approx 3.14 \cdot 10^{-17} \mathrm{~J}$. For these system sizes and surface coverage one has $\gamma=0.275 \mathrm{~J} / \mathrm{m}^{2}$ and, therefore, the respective contribution to the surface energy is $\gamma A_{c s} \approx 24.85 \cdot 10^{-17} \mathrm{~J}$. Comparing the contributions due to $\gamma$ and $\tau$ to the surface energy with each other, one finds that for the maximal line tension contribution one has 
$\pi d \tau / \gamma A_{c s} \approx 12.6 \%$. The minimal possible contribution due to $\tau$ to the surface energy will be accordingly three orders of magnitude lower.

In order to understand better the effect of the finite boundary between the island of standing thiols and laying-down ones on the measured surface tension let us consider a simple model of a $2 \mathrm{D}$ gas in the $\left(N, A_{c s}, T\right)$ ensemble. According to the condensation/evaporation theory of finite systems [163], the gas will exist as a homogeneous system up to some specific value of the $2 \mathrm{D}$ density, $\sigma^{*}$, after which it will be more favorable for the system to exist in an inhomogeneous state with the gas in coexistence with a condensated phase. It is possible to show that $\sigma^{*}$ is (i) system size-dependent and (ii) $\sigma^{*}>\sigma_{0 g}$, where $\sigma_{0 g}$ is the coexistence density of the less denser phase (gas) with the condensated phase of density $\sigma_{0 l}$. In the homogeneous phase the free energy cost due to the increase of the (2D) density above $\sigma_{0 g}$ is given by compression

$$
F_{\text {hom }}=\frac{A_{c s}}{2 k_{T}}\left(\sigma-\sigma_{0 g}\right)^{2},
$$

where $k_{T}$ is the $2 \mathrm{D}$ compressibility. On the other hand, if the excess amount of the gas (above $\sigma_{0 g}$ ) is used to create a noncompressible condensated phase the free energy cost is given by the line tension of the boundary between laying-down and standing thiols

$$
F_{\text {inh }}=2 \pi \tau R,
$$

where $\tau$ is the line tension and $R$ is the radius of the condensated phase. If to assume that the boundary between the two coexisting phases is sharp and the shape of the more denser phase is oval, one can obtain the radius of the condensated phase from a standard level rule $\sigma_{0 g}\left(A_{c s}-\pi R^{2}\right)+\sigma_{0 l} \pi R^{2}=N$ as

$$
R=\sqrt{\left(N-\sigma_{0 g} A_{c s}\right) /\left(\pi \Delta \sigma_{0}\right)}
$$

where $N$ is the total number of particles and $\Delta \sigma_{0}=\sigma_{0 l}-\sigma_{0 g} . \sigma^{*}$ is identified from the condition $F_{\text {hom }}=F_{\text {inh }}$ and equals to

$$
\sigma^{*}=\sigma_{0 g}+\left(\frac{16 \pi \tau^{2} k_{T}^{2} \sigma_{0 g}^{4}}{A_{c s} \Delta \sigma_{0}}\right)^{1 / 3}
$$


The above relation implies that the less denser system in a finite box will not immediately phase separate as $\sigma$ reaches the value of $\sigma_{0 g}$ but will rather stay in a homogeneous, though oversaturated $\left(\sigma>\sigma_{0 g}\right)$, state till $\sigma^{*}$ is reached. The oversaturated region shrinks with the system size, because $\sigma^{*}$ decreases with the increase of $A_{c s}$. This effect I also observe in my simulations. The lateral pressure, $P_{2 D}$, for the inhomogeneous system is given by

$$
P_{2 D}=-\frac{\partial F_{i n h}}{\partial A_{c s}}=\frac{\tau \sigma_{0 g} \sqrt{\pi}}{\sqrt{A_{c s} \Delta \sigma_{0}}} \frac{1}{\sqrt{\sigma-\sigma_{0 g}}} .
$$

This relation gives a rough correction due to the finite $2 \mathrm{D}$ boundary between the condensated and less denser phase to the surface tension of the reference bulk system. Giving this, one can represent the surface tension, $\gamma$, in the region where the less denser (layingdown thiols) and condensated phases (standing-up thiols) coexist as

$$
\gamma(\sigma)=\gamma_{p l}-\frac{\tau \sigma_{0 g} \sqrt{\pi}}{\sqrt{A_{c s} \Delta \sigma_{0}}} \frac{1}{\sqrt{\sigma-\sigma_{0 g}}},
$$

where $\gamma_{p l}$ is the constant level of the surface pressure at the coexistence. By fitting Eq. 4.18 to the surface tension values of the SC18 systems and $A_{c s}=903.5 \mathrm{~nm}^{2}$ in the coexistence range one obtains

$$
\begin{aligned}
\gamma_{p l} & =0.278505 \mathrm{~N} / \mathrm{m} \\
\frac{\tau \sigma_{0 g} \sqrt{\pi}}{\sqrt{A_{c s} \Delta \sigma_{0}}} & =0.00212309 \times 10^{-9} \mathrm{~N} / \mathrm{nm}^{2} .
\end{aligned}
$$

The respective fit follows perfectly the results obtained from the MD simulations (see Fig. 4.13). In the previous section the surface coverage of the laying-down, $\sigma_{0 g}=$ $0.806 \mathrm{~nm}^{-2}$, and standing-up SC18 thiols, $\sigma_{0 l}=4.17 \mathrm{~nm}^{-2}$, at the coexistence were identified. By using these values and Eq. $4.19 \mathrm{~b}$ one can estimate the line tension $\tau=8.2 \times 10^{-11} \mathrm{~N}$ between the laying-down thiols and the islands of the standig-up molecules.

To finalize the discussion of $\gamma-\sigma$ isotherms let us now take a look at the respective isotherm for the SC12 systems and $A_{c s}=903.5 \mathrm{~nm}^{-2}$, which is depicted in Fig. 4.14 . As is evident from the figure, my initialization and simulation procedures yield a very nice double-plateau pattern for these systems as well. The study of the oversaturated 


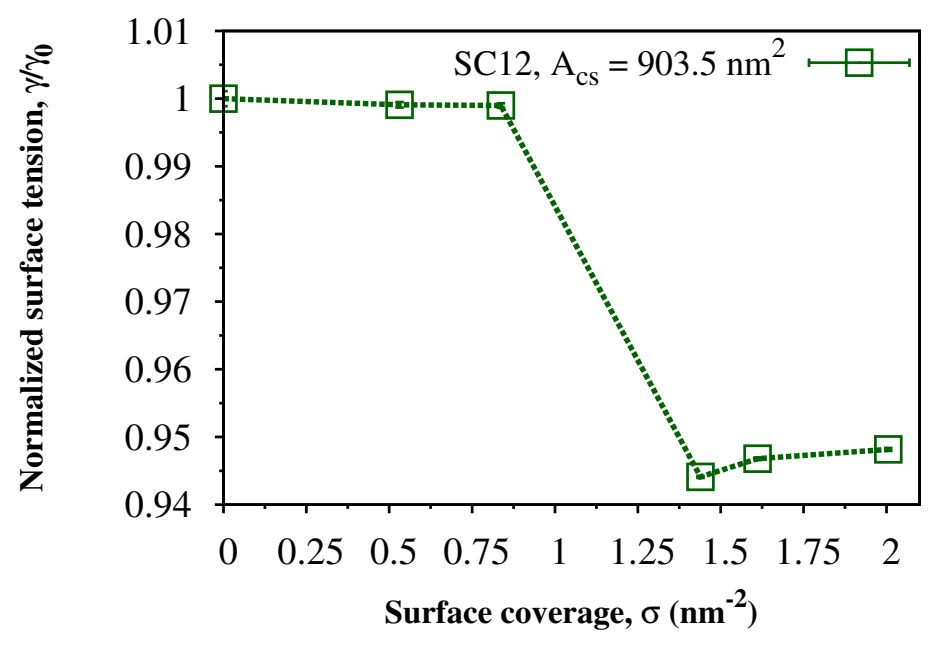

Figure 4.14: Normalized surface tension, $\gamma / \gamma_{0}$, for various values of dodecanethiol surface coverage, $\sigma \cdot \gamma_{0}$ is the surface tension of the bare surface of liquid mercury. Dashed line is the guide for the eyes.

region for the SC12 systems is omitted, because it was discussed (and the accompanying finite-size effects) in detail on the example of SC18 surfactants. For two simulated values of the dodecanethiol coverage at the partial filling of the laying-down monolayer $\left(\sigma=0.53124\right.$ and $\left.0.83671 \mathrm{~nm}^{-2}\right)$ the plateau at the surface tension level of the bare $\mathrm{Hg}$ film is observed. I expect this plateau to continue up to $\sigma=1.1156 \mathrm{~nm}^{-2}$, at which dodecanethiols reach the full coverage of the laying-down molecules (Tab. A.2). Subsequently, for higher values of $\gamma$ the surface tension comes down to the 2nd plateau, which corresponds to the coexistence of crystalline SC12 islands with the monolayer of the laying-down molecules.

\subsection{Structure in the islands of standing-up thiols}

\subsubsection{Molecular orientations}

After discussing the thermodynamic aspects of the thiol self-assembly on the surface of liquid mercury let us now take a detailed view on the structure of the crystalline islands of the standing thiol molecules. A sketch of a thiol molecules in the standing-up configuration on liquid $\mathrm{Hg}$ surface is shown in Fig. 4.15. The tilt angle, $\theta$, is defined 
as the angle between the $z$-axis and vector connecting the sulfur headgroup with the $\mathrm{CH}_{3}$ endgroup of the same thiol (i.e. thiol molecular axis). I chose the $x$-axis to point along the $\mathrm{S}-\mathrm{Hg}-\mathrm{S}$ bond of the $\mathrm{R}-\mathrm{S}-\mathrm{Hg}-\mathrm{R}-\mathrm{S}$ molecule under consideration. Then the azimuthal tilt direction angle, $\varphi$, is defined as the angle between the $x$-axis and the projection of the thiol molecular axis onto the $x y$-plane. Further, three vectors $\mathbf{R}_{\mathbf{n n} \mathbf{1}}$, $\mathbf{R}_{\mathbf{n n} \mathbf{2}}$ and $\mathbf{R}_{\mathrm{nn} \mathbf{3}}$ are defined as the vectors pointing from the bound $\mathrm{Hg}$ atom of the chosen $\mathrm{R}-\mathrm{S}-\mathrm{Hg}-\mathrm{R}-\mathrm{S}$ molecule to the 1 st, 2 nd and 3rd nearest bound $\mathrm{Hg}$ atoms. Consequently, I define three angles $\alpha_{1}, \alpha_{2}$ and $\alpha_{3}$ as the angles between the $x$-axis and vectors $\mathbf{R}_{\mathbf{n n} 1}$, $\mathbf{R}_{\mathbf{n n} 2}, \mathbf{R}_{\mathbf{n n} 3}$, respectively. The probability distribution of the tilt angles is given by

$$
P(\theta)=\frac{1}{N_{t h}}\left\langle\sum_{k}^{N_{t h}} \delta\left(\theta-\theta_{k}\right)\right\rangle, \text { with } \theta_{k}=\frac{180^{\circ}}{\pi} \arccos \frac{\left|l_{k_{z}}\right|}{\left|\mathbf{l}_{\mathbf{k}}\right|},
$$

where $\mathbf{l}_{\mathbf{k}}$ is the molecular axis vector of the $k$-th thiol and $l_{k_{z}}$ is its projection onto the $z$-axis, and it is treated $\langle\quad\rangle$ as average over molecular configurations. Additionally, the

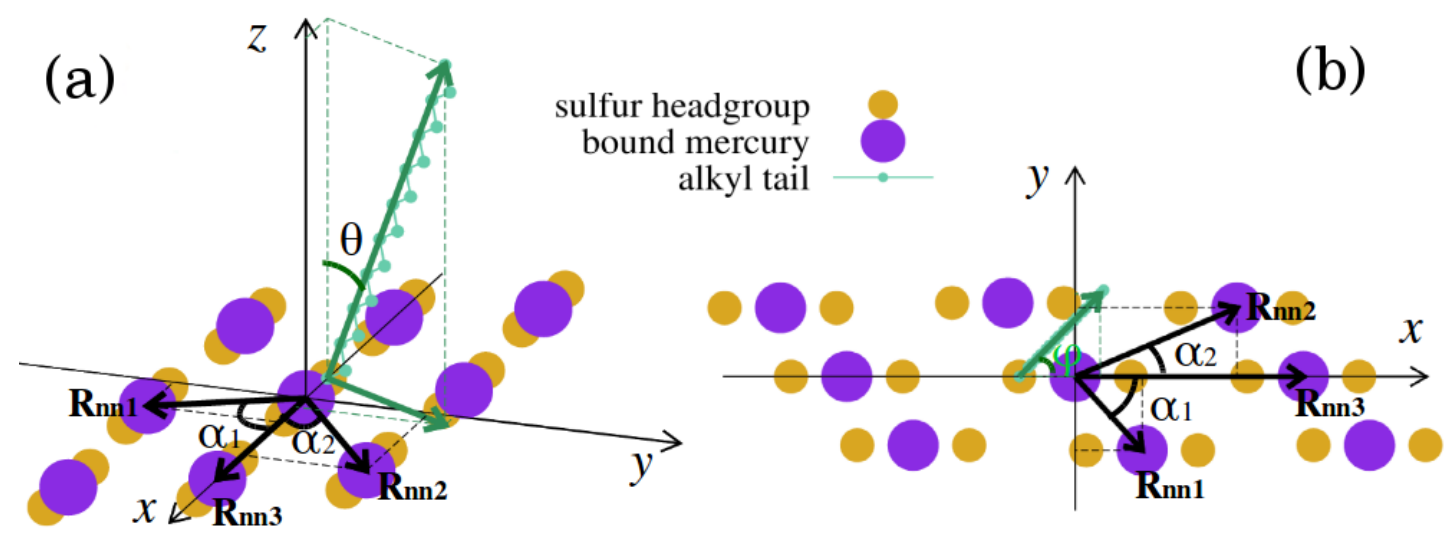

Figure 4.15: (a) Schematic view of an alkylthiol molecule in the standing phase on liquid $\mathrm{Hg} ; x$-axis is aligned along the $\mathrm{S}-\mathrm{Hg}-\mathrm{S}$ bond of a selected molecule; $\mathbf{R}_{\mathbf{n n} \mathbf{1}}, \mathbf{R}_{\mathbf{n n} \mathbf{2}}$ and $\mathbf{R}_{\mathbf{n n} 3}$ are $2 \mathrm{D}$ vectors connecting the selected bound $\mathrm{Hg}$ atom with its 1 st, 2nd and 3rd nearest neighbors, respectively; $\alpha_{1}, \alpha_{2}$ and $\alpha_{3}$ (not shown) are the angles between the $x$-axis and $\mathbf{R}_{\mathbf{n n} \mathbf{1}}, \mathbf{R}_{\mathbf{n n} \mathbf{2}}$ and $\mathbf{R}_{\mathbf{n n} \mathbf{3}}$ vectors, respectively. The tilt angle $\theta$ is defined as an angle between the molecular axis (the vector connecting $\mathrm{S}$ and $\mathrm{CH}_{3}$ groups of the same thiol) and its projection onto the $z$-axis. The azimuthal tilt direction angle $\varphi$ is defined as an angle between the projection of the molecular axis onto the $x y$-plane and the $x$-axis. (b) The $x y$-projection of the thiol molecule. The correct aspect ratio is preserved. 
surfactant conformations on top and bottom sides of the $\mathrm{Hg}$ film are treated as independent ones, thus, the total number of the molecular configurations used for the averaging equals two times the number of time steps. The distributions of the tilt angles for the dodecanethiol and octadecanethiol systems for $\sigma=1.6114 \mathrm{~nm}^{-2}$ and $A_{c s}=903.5 \mathrm{~nm}^{2}$ are represented in Fig. 4.16a. The observed tilt angles range from $35^{\circ}$ to $39^{\circ}$ for dodecanethiols and octadecanethiols, respectively. The broad peaks at $90^{\circ}$ in the distributions of the tilt angles are due to the laying-down molecules. The broadness of these peaks is caused by the surface corrugations due to the capillary waves, the amplitude of which may reach several Angströms for such system cross-section and room temperature, as well as by occasional folding of the laying-down molecules over each other.

To proceed with the further analysis the following criteria is introduced. A R-S-Hg$\mathrm{R}-\mathrm{S}$ molecule will be considered to be a standing one, if the tilt angle of its both thiol tails is lesser than $45^{\circ}$. Then $N_{m}^{i s l}$ is defined as the total number of standing molecules ( $\mathrm{R}-\mathrm{S}-\mathrm{Hg}-\mathrm{S}-\mathrm{R})$ for an instant molecular configuration, i.e. $N_{m}^{i s l}$ varies with time. Now to check the mutual azimuthal tilt orientation of thiols the distribution of the mutual azimuthal tilt direction of the two thiols belonging to the same $\mathrm{R}-\mathrm{S}-\mathrm{Hg}-\mathrm{S}-\mathrm{R}$ complex is calculated as

$$
P(\omega)=\left\langle\frac{1}{N_{m}^{i s l}} \sum_{m}^{N_{m}^{i s l}} \delta\left(\omega-\omega_{m}\right)\right\rangle, \text { with } \omega_{m}=\frac{180^{\circ}}{\pi} \arccos \frac{\mathbf{l}_{\mathbf{1}} \cdot \mathbf{l}_{\mathbf{2}}}{\left|\mathbf{l}_{\mathbf{x} \mathbf{y}}\right|\left|\mathbf{l}_{\mathbf{2}}\right|},
$$

where $\mathbf{l}_{\mathbf{x y}}$ and $\mathbf{l}_{\mathbf{x y}}$ are the $x y$-projections of the molecular axes of two thiols of the same $\mathrm{R}-\mathrm{S}-\mathrm{Hg}-\mathrm{S}-\mathrm{R}$ molecule. Additionally, the mutual orientation of the $\mathrm{S}-\mathrm{Hg}-\mathrm{S}$ bonds for the surfactants lying within $10 \AA$ (corresponds to three coordination spheres of bound $\mathrm{Hg}$ atoms) from each other is calculated as

$$
P(\xi)=\left\langle\frac{1}{N N_{t o t}} \sum_{m}^{N_{m}^{i s l}} \sum_{\substack{n>m, r_{m n}<10 \AA ⿻}}^{N_{m}^{i s l}} \delta\left(\xi-\xi_{m n}\right)\right\rangle, \text { with } \xi_{m n}=\frac{180^{\circ}}{\pi} \arccos \frac{\mathbf{h}_{\mathbf{m}} \cdot \mathbf{h}_{\mathbf{n}}}{\left|\mathbf{h}_{\mathbf{m}}\right|\left|\mathbf{h}_{\mathbf{n}}\right|}
$$

where $\mathbf{h}_{\mathbf{m}}$ is the two-dimensional (2D) vector of the $\mathrm{S}-\mathrm{Hg}-\mathrm{S}$ bond belonging to the $m$-th $\mathrm{R}-\mathrm{S}-\mathrm{Hg}-\mathrm{S}-\mathrm{R}$ molecule, $r_{m n}$ is the distance (in the $x y$-plane) between the $m$-th and $n$-th bound $\mathrm{Hg}$ atoms, and $N N_{\text {tot }}$ is the total number of bound $\mathrm{Hg}$ atom pairs that lie within the sphere of the radius of $10 \AA$ for a given instant molecular configuration. The $P(\omega)$ 


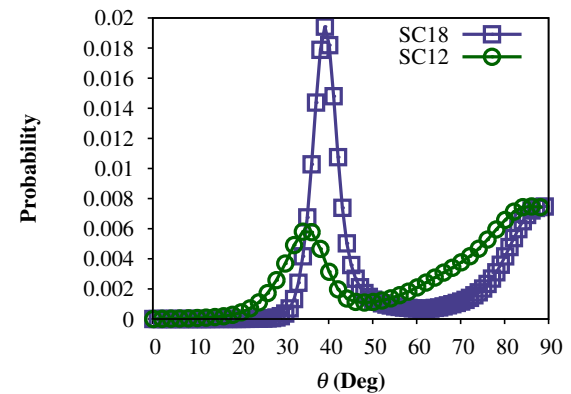

(a)

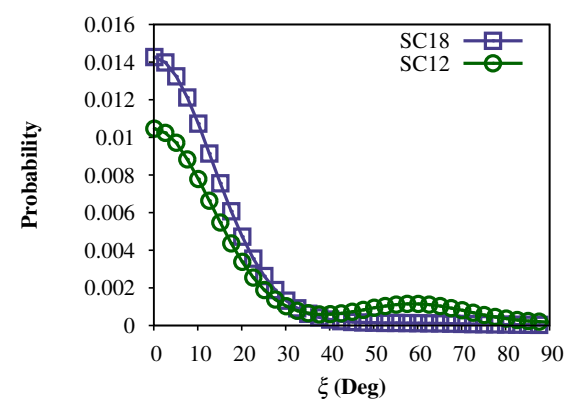

(c)

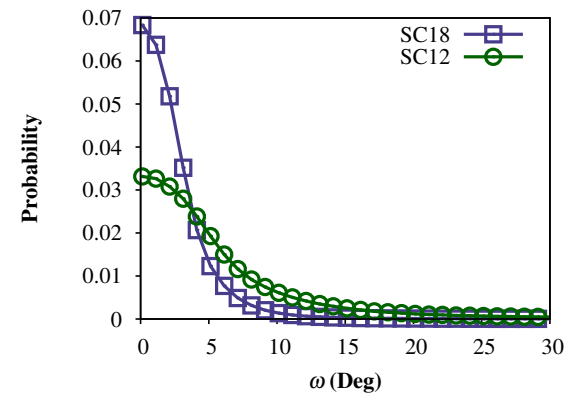

(b)

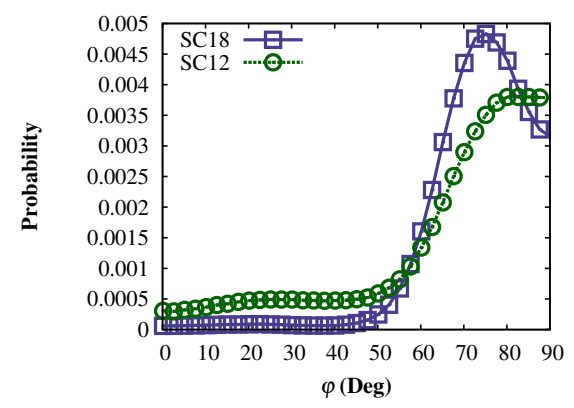

(d)

Figure 4.16: Molecular orientations in the islands of standing octadecanethiol (SC18) and dodecanethiol (SC12) molecules. $\sigma=1.6114 \mathrm{~nm}^{-2}$ and $A_{c s}=903.5 \mathrm{~nm}^{2}$. (a) Distribution of the tilt angles, $P(\theta)$ (Eq. 4.20); (b) distribution of the mutual azimuthal tilt direction of the thiols of the same R-S-Hg-S-R surfactant complex, $P(\omega)$ (Eq. 4.21); (c) distribution of the mutual orientation of the $\mathrm{S}-\mathrm{Hg}-\mathrm{S}$ bonds of the neighboring surfactants, $P(\xi)(\mathrm{Eq}$. 4.22); (d) distribution of the azimuthal tilt directions relative to the $\mathrm{S}-\mathrm{Hg}-\mathrm{S}$ bonds, $P(\varphi)(\mathrm{Eq} .4 .23$.

and $P(\xi)$ distributions (for both, SC18 and SC12 molecules) are shown in Fig. 4.16b and $4.16 \mathrm{c}$, respectively. From these distributions one can see that in the SC18 as well as $\mathrm{SC} 12$ islands of the standing thiols are tilted in the same direction and the $\mathrm{S}-\mathrm{Hg}-\mathrm{S}$ bonds of the neighboring surfactants are aligned. From broader peaks of the SC12 systems one can infer that the SC12 islands possess larger degree of orientational disorder. In order to find out the azimuthal tilt direction of thiols relative to the $\mathrm{S}-\mathrm{Hg}-\mathrm{S}$ bonds, I compute the respective distribution

$$
P(\varphi)=\left\langle\frac{1}{2 N_{m}^{i s l}} \sum_{m}^{N_{m}^{i s l}} \sum_{i=1}^{2} \delta\left(\varphi-\varphi_{m i}\right)\right\rangle, \text { with } \varphi_{m i}=\frac{180^{\circ}}{\pi} \arccos \frac{\mathbf{l}_{\mathbf{i}_{\mathbf{x y}}} \cdot \mathbf{h}_{\mathbf{m}}}{\left|\mathbf{l}_{\mathbf{i}_{\mathbf{x y}}}\right|\left|\mathbf{h}_{\mathbf{m}}\right|}
$$


where $\mathbf{l}_{\mathbf{i}_{\mathbf{x y}}}$ is the $x y$-projection of the molecular axis of the $i$-th thiol belonging to the $m$-th R-S-Hg-S-R surfactant. The $P(\varphi)$ distribution is shown in Fig. 4.16d, This distribution reveals that the thiols tilt direction creates an angle of $\sim 75^{\circ}$ and $81-83^{\circ}$ with the $\mathrm{S}-\mathrm{Hg}-\mathrm{S}$ bonds in the case of SC18 and SC12 systems. The pronounced tail of the $P(\varphi)$ distribution of the SC12 systems confirms the above suggestion of the larger degree of orientational disorder for these systems.

\subsubsection{Packing of the headgroups}

In order to study the packing of the headgroups I first compute the 2D radial pair distribution function of the bound mercury atoms, $g(r)$, as

$$
g(r)=\left\langle\frac{R_{\max }^{2}}{2 r \Delta r N_{\text {pairs }}} \sum_{m}^{N_{m}^{i s l}} \sum_{\substack{n>m, r_{m n}<R_{\max }}}^{N_{m}^{i s l}} \delta\left(r-r_{m n}\right)\right\rangle
$$

where $r_{m n}$ is the distance in the $x y$-plane between two bound $\mathrm{Hg}$ atom belonging to the $m$-th and $n$-th surfactant molecules, $R_{\max }=30 \AA$ is the maximum distance, within which $g(r)$ is calculated, $\delta r=0.01 \AA$ is the bin size used to calculate the $g(r)$, and $N_{\text {pairs }}$ is the total number of bound $\mathrm{Hg}$ atoms, the distance between which does not exceed $R_{\max }$ for a given molecular configuration. Here I assume that, despite of the finite extensions of the standing-up thiol islands, thus calculated $g(r)$ gives a correct estimate of the 1st, 2nd and 3 rd coordination spheres. Moreover, we will see in the next chapter that the locations of these coordination spheres for the bound $\mathrm{Hg}$ in the thiol islands agrees very well with the perfectly periodic systems of standing-up thiols. Fig. 4.17 demonstrates the radial distribution functions for the octadecanethiol and dodecanethiol systems. One can see that for these systems the headgroups pack virtually identical. The distance to the 1st, 2nd and 3rd nearest neighbors equals to 5.82, 8.2, $9.4 \AA$ and 5.82, 8.28, $9.32 \AA$ for the SC18 and SC12 molecules, respectively. The distances to the 1st and 2nd neighbors give the lengths of the base vectors of the respective headgroup unit cell and the distance to the 3rd nearest neighbors corresponds to the length of the shortest diagonal of the unit cell. The $i$-th coordination sphere is defined to lie in the interval $\left(R_{i-1}, R_{i}\right]$, where $R_{0}=0$, $R_{1}=7.1, R_{2}=8.8$ and $R_{3}=10 \AA$ in both cases of SC18 and SC12 thiols. Subsequently, the angular distributions, $P\left(\alpha_{i}\right)$, of the neighboring bound $\mathrm{Hg}$ atoms in the $1 \mathrm{st}$, 2nd and 


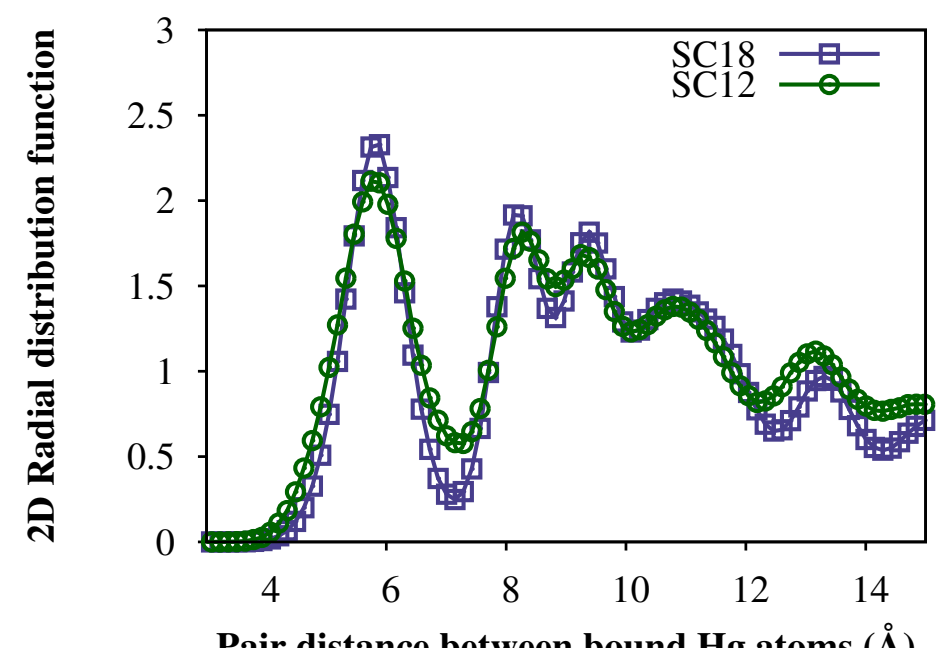

Figure 4.17: Radial distribution function of bound $\mathrm{Hg}$ atoms for octadecanethiol and dodecanethiol molecules in the standing-up phases. $\sigma=1.6114 \mathrm{~nm}^{-2}$ and $A_{c s}=$ $903.5 \mathrm{~nm}^{2}$.

3rd coordination spheres was calculated as

$$
P\left(\alpha_{i}\right)=\left\langle\frac{1}{N N_{i}} \sum_{m}^{N_{m}^{i s l}} \sum_{\substack{n>m, R_{i-1}<r_{m n} \leq R_{i}}}^{N_{m}^{i s l}} \delta\left(\alpha_{i}-\alpha_{m n}\right)\right\rangle \text {, with } \alpha_{m n}=\frac{180^{\circ}}{\pi} \arccos \frac{\mathbf{h}_{\mathbf{m}} \cdot \mathbf{r}_{\mathbf{m n}}}{\left|\mathbf{h}_{\mathbf{m}}\right|\left|\mathbf{r}_{\mathbf{m n}}\right|}
$$

where $i=1,2,3$, and $N N_{i}$ is the total number of pairs of the bound $\mathrm{Hg}$ atoms with distances corresponding to the $i$-th coordination sphere for the particular surfactant configuration. This allowed us to determine the relative locations of the bound $\mathrm{Hg}$ atoms in the unit cell and, consequently, the angle between the the base vectors, which is simply $\alpha_{1}+\alpha_{2}$. The angular distributions for the 1st, 2nd and 3rd nearest neighbors are show in Figs. 4.18a, 4.18b and 4.18c, respectively. One sees that these distributions peak at almost the same positions for the SC18 and SC12 systems, yielding the angle between the base vectors of $97.22^{\circ}$ and $97.58^{\circ}$ for the SC18 and SC12 thiol islands, respectively. This results in the unit cell area of $47.3498 \AA^{2}$ for octadecanethiols, which, giving two thiols per unit cell, yields the SC18 surface coverage of $4.22 \mathrm{~nm}^{-2}$. Fig. $4.18 \mathrm{~d}$ demonstrates the zoomed in structure of the headgroups in the island of standing SC18 molecules with the outlined resulting unit cell. More pure, free of boundary effects, 


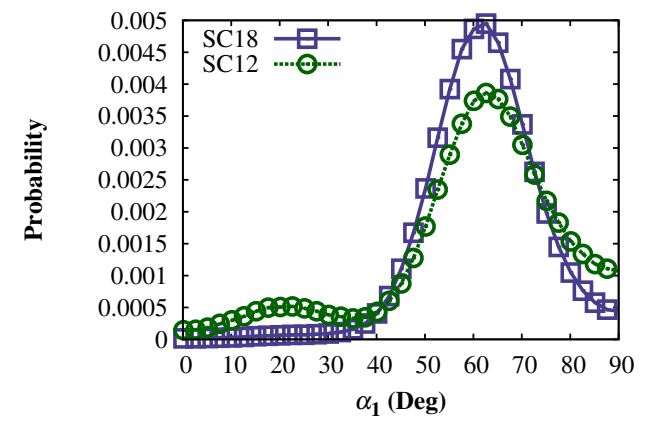

(a)

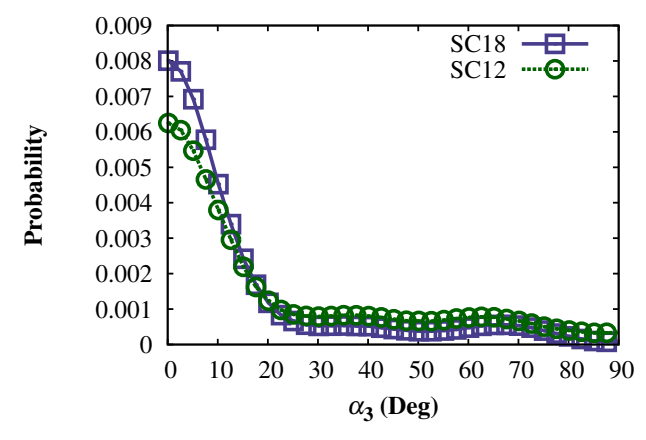

(c)

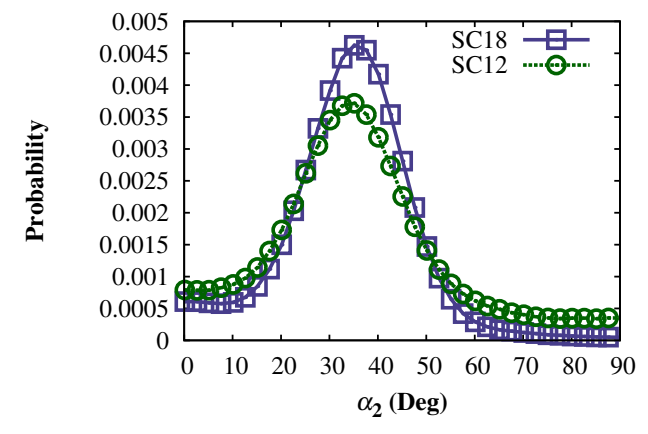

(b)

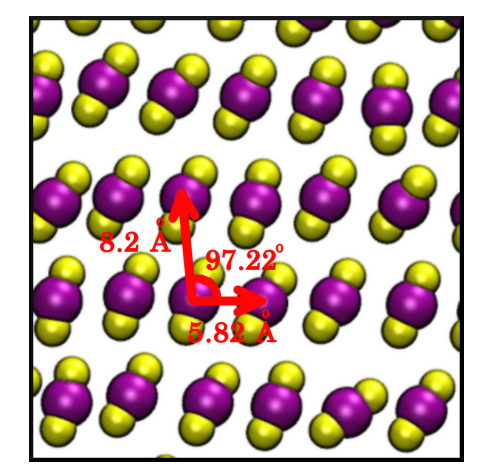

(d)

Figure 4.18: Angular distributions of the bound $\mathrm{Hg}$ atoms in the 1st (a), 2nd (b) and 3rd (c) coordination spheres (Eq:4.25). Zoomed view of the headgroup packing in the high-density crystalline thiol islands (d). $\sigma=1.6114 \mathrm{~nm}^{-2}$ and $A_{c s}=903.5 \mathrm{~nm}^{2}$

results for the structure of the thiol crystalline phases on liquid $\mathrm{Hg}$ will be obtained in the next chapter, where I utilize the Molecular Dynamics simulations in the constant temperature-stress tensor ensemble in order to study ideally periodic crystalline systems.

\subsection{Discussions and conclusions}

In this chapter I have studied the self-assembly of alkylthiols, namely of octa- and dodecanethiols, on the surface of liquid mercury at $T=293 \mathrm{~K}$. The observed coexistence of laying-down molecules with the crystalline islands of standing-up thiols is consistent with the available experimental data [62, 64, 65]. The molecules in the laying-down conformations exhibit some small patches of approximately 7-10 thiols with aligned 
molecular axes. These patches of laying-down molecules are randomly oriented and, generally, unlike from the similar systems on gold [6], no long-range ordering of those domains is observed. Furthermore, the "aggregated" 2D phase of laying-down thiols in the low-coverage regime (rather than the $2 \mathrm{D}$ gas phase conjectured from simple fits to the experiments) was found in the Molecular Dynamics simulations presented in this section.

The thiol packing in the crystalline islands is the following. The bound mercury and sulfur headgroups build up an oblique 2D lattice with one bound $\mathrm{Hg}^{*}$ and two $\mathrm{S}$ atoms per unit cell. The magnitude of the base vectors and the resulting thiol surface coverage in the islands are very close to those for the rectangular unit cell proposed in the X-Ray experiments [64]. As was discussed in the introduction the possibility of the oblique packing of the headgroups has been also mentioned in the experiments [65]. The angle between the base vectors of the oblique lattice obtained in the simulations differs only by $\sim 7^{\circ}$ from the rectangular unit cell. It may be difficult to distinguish such small deviations from the rectangular unit cell in the X-Ray spectra of the thiol SAMs on liquid mercury. Additional data on the headgroup packing from other experimental techniques is needed to clear this point. The obtained collective tilt angles of $35^{\circ}$ and $39^{\circ}$ for SC18 and SC12 thiols generally overestimate the tilt angle of $27^{\circ}$ extracted from the X-Ray data [64]. Possible reasons for this deviation are the following: (i) the structure of the obtained in the simulations crystalline islands due to the finite sizes does not fully correspond to the macroscopic systems studied in the experiments; (ii) in the experiments the $\mathrm{Hg}$ surface was assumed to be flat (i.e. no capillary waves) for the estimation of the tilt angles; (iii) the thiol monolayer was assumed to have no defects in the experiments. We have seen in Sec. 4.1.2 that various long-living defects (such as spiral thiol islands and grain boundaries between the patches of standing-up thiols with different collective tilt directions) can emerge in the simulations. Persistent spiral islands of surfactant molecules have been also previously seen in the simulations of the self-assembly on the surfaces of gold [34] and water [68, 69].

If a mercury droplet with a thiol SAM covering it is mechanically compressed too strongly, it will expand its surface area, which will then become accessible to the thiol monolayer. In such case the monolayer can drastically change its phase behavior and transform into a laying-down phase, which will, in turn, uncover the patches of the bare $\mathrm{Hg}$ surface. Such situation would be critical for organic electronics applications and 
lead to a short circuit, since the properties of a metal-SAM-metal junctions are tuned by mechanically varying the shape of the mercury drop-electrode [51].

My simulation model, with thiols being chemisorbed in the laying-down as well as in the standing-up conformations, has allowed me to recover experimentally observed coexistence of the crystalline islands of the standing-up thiols with their laying-down counterparts, whereas on $\mathrm{Au}$ the laying-down conformations are generally referred to the physisorbed surfactants [6]. This may suggest that the initial stage of the thiol adsorption onto liquid $\mathrm{Hg}$, the physisorption, does not last very long or is absent at all and the chemisorption happens much faster compared to the adsorption onto gold. In turn, this may be explained by assuming that an appropriate mutual orientation of the electronic orbitals of mercury and sulfur atoms is more probable to be achieved than of gold and sulfur atoms due to the high mobility of liquid mercury. This assumption is strongly supported by the optical tensiometry results discussed in the introduction, where a twostage adsorption model involving physi- and chemisorption of thiols was not able to to explain the observations from the experiments, and, instead, the simpler one-stage diffusion-limited Langmuir adsorption model was more attractive for the elucidation of the mechanism of the thiol adsorption onto mercury [27]. 


\section{5 | In-depth study of densely packed alkylthiol phases on liquid mercury}

In the previous chapter the self-assembly of the alkylthiol monolayers on liquid $\mathrm{Hg}$ with growing surface coverage of thiols was characterized. We saw the emergence of highly ordered and densely packed islands of thiols standing tilted to the surface normal after the layer of the laying-down molecules was completely filled. Here I would like to focus on the resulting crystalline phases of alkylthiols on the surface of liquid mercury, since these phases are used in the majority of applications [50]. In the next section the simulation details are presented. Subsequently, the effect of temperature on the structure of the monolayer is studied. The temperature, at which the ordered thiol SAM loses its crystalline structure, is estimated. Next, the effect of the absence of the $\mathrm{S}-\mathrm{Hg}-\mathrm{S}$ bond on the structure of the monolayer at $T=293 \mathrm{~K}$ is demonstrated. Afterwards, the effects of the length of the alkylthiol molecules and of the lateral compression of the SAM are explored. In order to finalize the discussion, the influence of the interaction strength between the bound mercury atoms (free parameter of my model) is analyzed. Physically the increase of this parameter yields higher lateral molecular packings within the SAM of thiols. This enables me to gain insights into the respective transformations in the monolayer upon the rise of the molecular coverage above the one of $\sim 4.22 \mathrm{~nm}^{-2}$ inside the crystalline octadecanethiol island. The results are discussed in detail and compared with the literature where possible. 


\subsection{Simulation details}

In this chapter MD simulations in the ensemble with constant stress tensor and temperature $(N \mathcal{P} T)$ are performed in order to study the structure of the dense crystalline alkylthiol SAMs in addition to the simulations in the NVT ensemble from the previous chapter for the surfactants with the $\mathrm{S}-\mathrm{Hg}-\mathrm{S}$ bond (see Fig. $1.7 \mathrm{a}$ ). For the study of temperature effects the octadecanethiol (SC18) SAM on $\mathrm{Hg}$ is chosen as a representative example. The equilibrated SC18 system at $T=293 \mathrm{~K}$ for $\sigma=1.6114 \mathrm{~nm}^{-2}$ and $A_{c s}=903.5 \mathrm{~nm}^{2}$ was the initial configuration for the simulations at higher temperatures. First, the system for $T=310 \mathrm{~K}$ has been prepared by increasing the temperature stepwise from $293 \mathrm{~K}$ to $310 \mathrm{~K}$ during $4 \mathrm{~ns}$ of the simulation time. Afterwards the system was let to evolve at $310 \mathrm{~K}$ and no drift of the total energy was observed. Likewise, the system at $T=330 \mathrm{~K}$ was prepared by taking the equilibrium configuration at $T=310 \mathrm{~K}$ and subsequently increasing the temperature from $310 \mathrm{~K}$ to $330 \mathrm{~K}$ in the course of $4 \mathrm{~ns}$ of the simulation time. Then, in order to reach equilibrium the system required another $20 \mathrm{~ns}$ of simulation time at $T=330 \mathrm{~K}$. In analogy to this, for $T=350 \mathrm{~K}$ I have started with the equilibrium configuration obtained for $T=330 \mathrm{~K}$ and step-by-step increased the temperature to $350 \mathrm{~K}$ in $4 \mathrm{~ns}$. After this, one could still see partially melted crystalline islands of octadecanethiols on both sides of the $\mathrm{Hg}$ film. But after another $24 \mathrm{~ns}$ of simulations both islands were completely destroyed and no ordered thiol structures on mercury were observed. In all the cases (apart from $T=350 \mathrm{~K}$ ) the total simulation time was taken to be $107 \mathrm{~ns}$ and the measurements were performed every $10 \mathrm{ps}$ as in the previous chapter. The force fields are given in Sec. 4.1.1.

The system of choice for the study of the molecular morphology effects is again the SC18 SAM at $T=293 \mathrm{~K}$ for $\sigma=1.6114 \mathrm{~nm}^{-2}$ and $A_{c s}=903.5 \mathrm{~nm}^{2}(1156$ thiol molecules per side of the $\mathrm{Hg}$ film), but in this case the surfactants do not have the S$\mathrm{Hg}-\mathrm{S}$ bond, i.e. only a single thiol is attached to a single mercury atom (see Fig. $1.7 \mathrm{~b}$ ). Such a surfactant complex will be called a single-tailed surfactant in the following. A surfactant with the $\mathrm{S}-\mathrm{Hg}-\mathrm{S}$ bond will be called a double-tailed surfactant. The number of the bulk mercury atoms is taken such that the total number of all mercury atoms corresponds to the respective system with the double-tailed surfactants. This system was initialized with a preset crystalline island of upright surfactants in analogy to the way described in Sec. 4.1.2. It took around 4 ns to equilibrate the system, which is 
considerably faster than in the respective simulations with the double-tailed surfactants. The short relaxation times are simply explained by the shorter overall length of the single-tailed surfactants compared to the double-tailed ones. The total simulation time and sampling interval were also $107 \mathrm{~ns}$ and $10 \mathrm{ps}$, respectively.

The simulation of the crystalline SAMs of the standing-up thiols of various chain lengths were performed in the $N \mathcal{P} T$ ensemble (described in Chap. 2) at the full coverage of the standing-up thiols $\left(\sim 4.22 \mathrm{~nm}^{-2}\right)$ that form $\mathrm{S}-\mathrm{Hg}-\mathrm{S}$ bonds with mercury (see Fig. 1.7 a). The total number of alkylthiol molecules per side of the $\mathrm{Hg}$ film was 432 for all the simulations in the $N \mathcal{P} T$ ensemble. The lateral pressure was fixed at $-273 \mathrm{~atm}$ (coexistence pressure of the laying-down and standing-up SC18 phases) in the $x$ - and $y$-direction independently. The negative lateral pressure simply means that it acts towards the center of the SAM from each side. The off-diagonal elements of the pressure tensor, $\mathcal{P}$, were set to zero independently as well. The normal stress component was left unconstrained fluctuating around zero, since there was an empty space above and below the film. The simulation box had a slab geometry with the $\mathrm{Hg}$ film in the middle of it (Fig. 4.3). An equal number of surfactants (with $\mathrm{S}-\mathrm{Hg}-\mathrm{S}$ bonds) was placed from both sides of the $\mathrm{Hg}$ film according to the rectangular packing (two alkylthiols per unit cell) just as described in Sec. 4.1.2. The equilibration time in this case was $8.4 \mathrm{~ns}$. The total simulation time was at least $230 \mathrm{~ns}$ and the sampling was done every $20 \mathrm{ps}$. The compression simulations were performed for the SC18 systems. The starting point for the simulations at higher lateral pressure (in the modulo sense) is the equilibrated configuration for the lateral pressure $P=-273$ atm obtained from the simulation of the SC18 SAM (with S-Hg-S bond) at the full coverage of the standing-up thiols described above in the previous passage. Starting from this configuration the lateral pressure in $x$ - and $y$-directions was stepwise decreased to $-285 \mathrm{~atm}$ during the course of $8 \mathrm{~ns}$ of simulations, after which the SAM at $-285 \mathrm{~atm}$ was evolved another $60 \mathrm{~ns}$ to reach an equilibrium. Likewise, the equilibrium systems at lower values of the lateral pressure (stronger compression) were obtained from the equilibrated preceding states. The total simulation time and sampling interval were $207 \mathrm{~ns}$ and $20 \mathrm{ps}$, respectively.

The systems for the simulations at various strength of the $\mathrm{Hg}^{*}-\mathrm{Hg}^{*}$ interaction were set up and simulated in analogy to the simulations of the chain length effects.

For all the system simulated in this chapter periodic boundary conditions were applied in all the directions and the time-steps were taken the same as in the previous 
chapter.

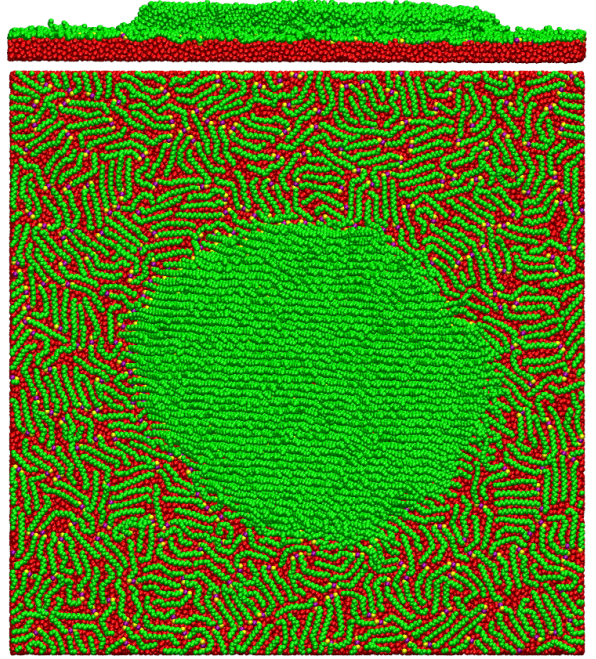

(a) $T=293 \mathrm{~K}$

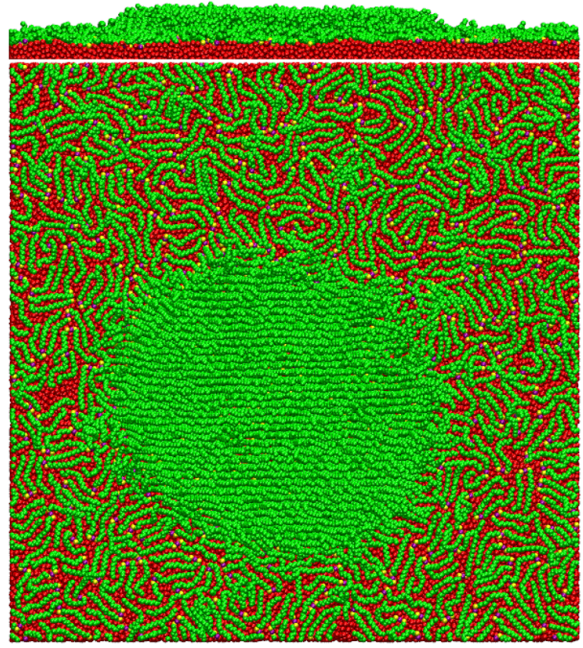

(c) $T=330 \mathrm{~K}$

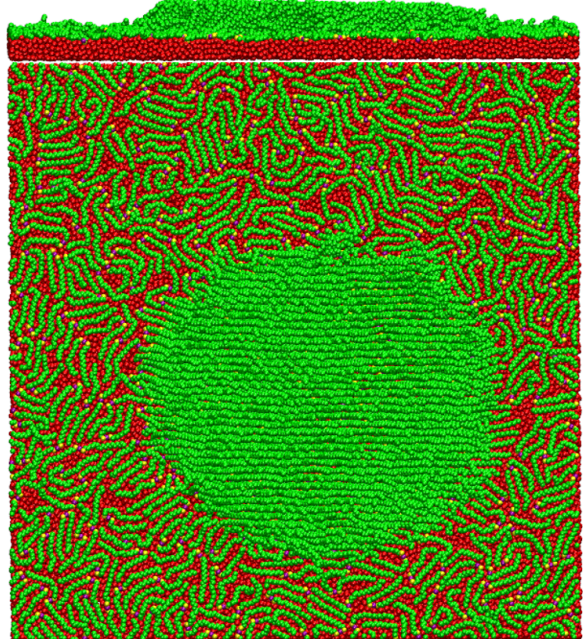

(b) $T=310 \mathrm{~K}$



(d) $T=350 \mathrm{~K}$

Figure 5.1: Snapshots of the crystalline island of octadecanethiol at various temperatures for $\sigma=1.6114 \mathrm{~nm}^{-2}$.

\subsection{Temperature effects}

Fig. 5.1 demonstrates the system snapshots at various temperatures. One sees that the crystalline island of standing thiols stays quite stable over the temperature range be- 
tween 293 and $330 \mathrm{~K}$. It only slightly shrinks such that at $T=330 \mathrm{~K}$ there is $47 \%$ of molecules in the standing-up conformations compared to $51 \%$ at the room temperature. Additionally, one sees that the island at $330 \mathrm{~K}$ has a less pronounced boundary and much more disturbed collective thiol orientations compared to the one at $293 \mathrm{~K}$. From the distribution of the tilt angle at different temperatures (Fig. 5.2) one can clearly see that the average tilt angle (the angle between the $z$-axis and molecular axis) decreases as the temperature grows. At $T=330 \mathrm{~K}$ the average tilt angle is about $37^{\circ}$ compared to $39^{\circ}$ for $293 \mathrm{~K}$. The height of the peaks of the tilt angle distribution decreases with temperature as well, indicating the increase in the rotational disorder. The azimuthal tilt direction angle (relatively to the $\mathrm{S}-\mathrm{Hg}-\mathrm{S}$ bond) also increases towards $90^{\circ}$ upon the rise in temperature (Fig. 5.2b). The lower 1st and 2nd peaks of the 2D radial distribution function of the bound mercury atoms at higher temperatures indicate a slight increase in the translational disorder, but, apart from this, the packing of the headgroups is not considerably influenced in the temperature region between 293 and $330 \mathrm{~K}$ (Fig. 5.3a). This observation is also confirmed by the angular distributions of the nearest neighbors (Figs. 5.3b $5.3 \mathrm{~d}$ ). In all the cases the positions of the peak maxima remain approximately the same, but the peak heights drop down as the temperature rises.

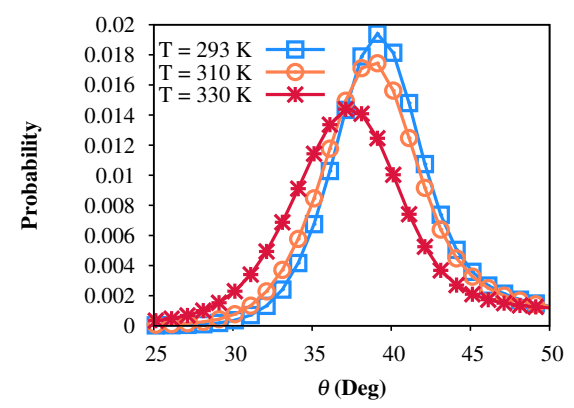

(a)

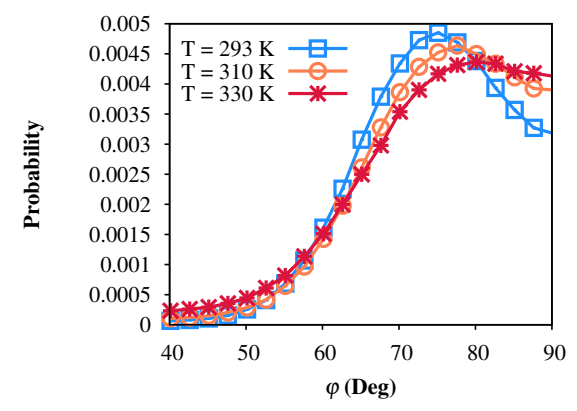

(b)

Figure 5.2: Distribution of the tilt (a) and azimuthal tilt direction (b) angles for the crystalline island of octadecanethiols at various temperatures. 


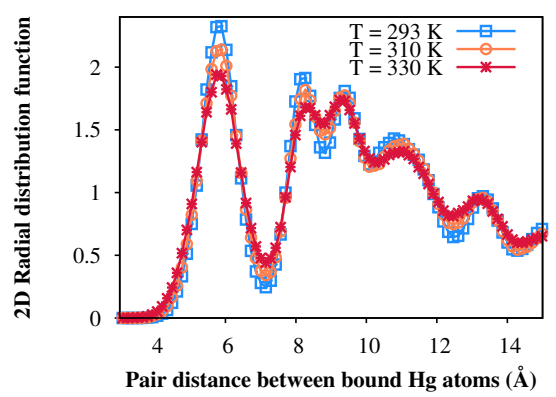

(a)

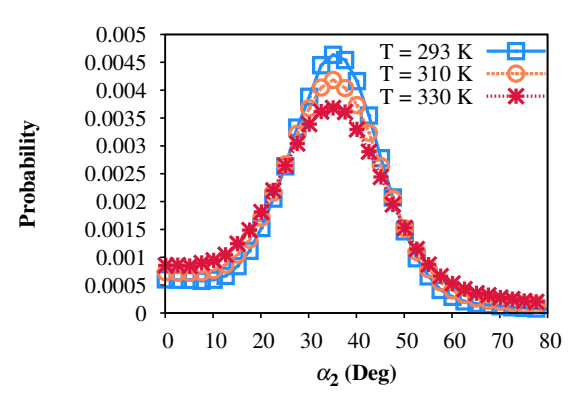

(c)

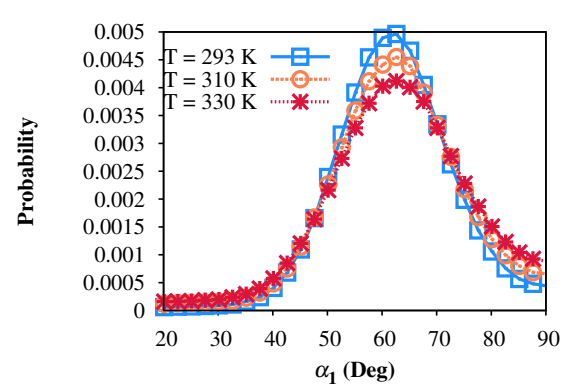

(b)

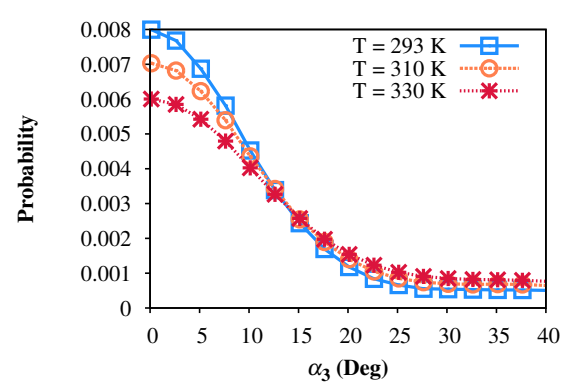

(d)

Figure 5.3: Temperature influence on the packing of the headgroups. Radial distribution function of $\mathrm{Hg}^{*}$ atoms (a); and angular distributions of the 1st (b), 2nd(c) and 3rd (d) nearest neighbors.

\subsection{Influence of molecular morphology: case of the sur- factants without S-Hg-S bond}

In the previous sections we have seen that various thiol phases (such as the monolayer of the laying-down thiols as well as crystalline islands of standing-up thiols) may emerge and coexist on the surface of liquid mercury as the function of thiol surface coverage. As a single surfactant unit I chose a molecular complex consisting of two thiol tails attached via chemical bonds to a single mercury atom as shown in Fig. 1.7 A. (double-tailed surfactant). Yet another, maybe somewhat less realistic scenario would be a surfactant with a single thiol molecule attached to a mercury atom as shown in Fig. 1.7p (singletailed surfactant). Below such a scenario is evaluated in detail.

Fig. 5.4 shows the comparison of the molecular conformations for the systems with double- and single-tailed surfactants. As one can see, the system with a single thiol 


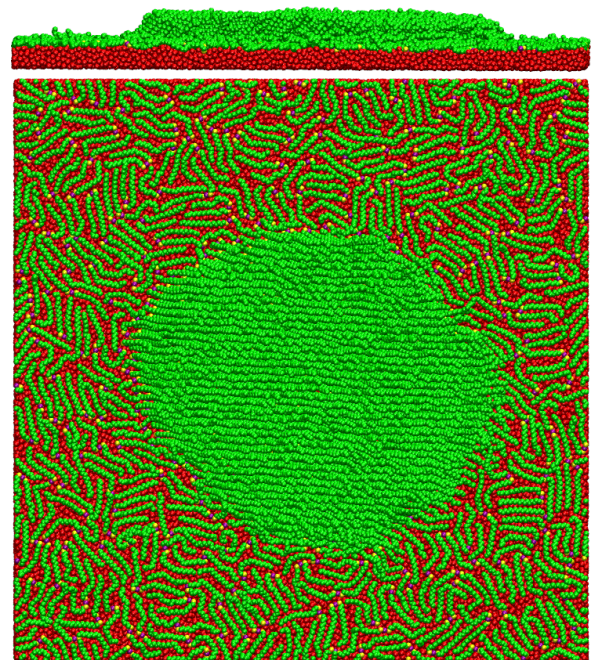

(a)

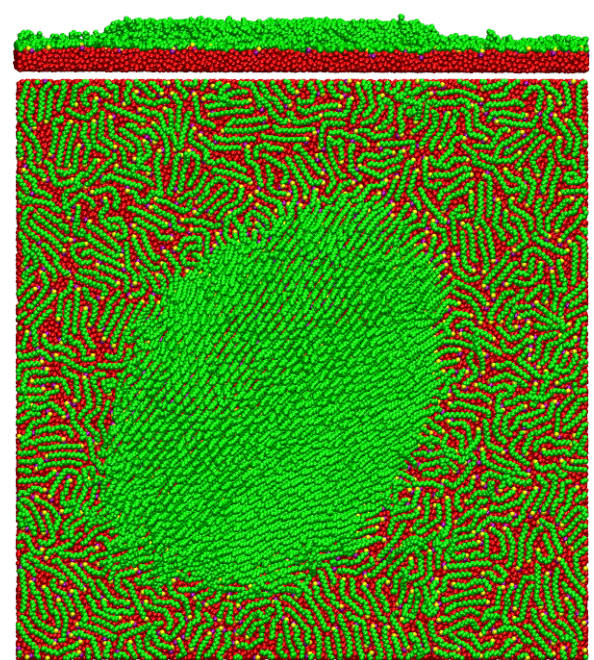

(b)

Figure 5.4: Comparison of molecular conformations for the case of double-tailed (a) and single-tailed (b) surfactants. $\sigma=1.6114 \mathrm{~nm}^{-2}, A_{c s}=903.5 \mathrm{~nm}^{2}, T=293 \mathrm{~K}$.

molecule per bound mercury atom features a stable island of standing-up thiols as well. The shape of this island is, however, much more extended along the direction of the collective tilt angle of the single-tailed surfactants. The comparison of the tilt angle distributions for both systems is depicted in Fig. 5.5. The tilt angle distribution of the single-tailed surfactants has a peak at about $45.4^{\circ}$, which is noticeably larger compared to $39^{\circ}$ for the system that has two thiols per surfactant. A low peak height in the tilt angle distribution of the single-tailed surfactants also indicates a lager degree of orientational disorder in the single-tailed system compared to the double-tailed one. The packing of the bound mercury atoms in the island of the standing-up single-tailed surfactant molecules is also strongly disturbed compared to the double-tailed systems (compare Figs. 5.6a and 4.18d). This is also confirmed by the respective radial distribution function, which is rather reminiscent of some liquid order (Fig. 5.6b) compared to the pronounced crystalline ordering in the system with the double-tailed surfactants (recall Figs. 4.17 and 4.18d). The first peak of the radial distribution of the bound $\mathrm{Hg}^{*}$ atoms has a much lower amplitude compared to the second one, but the peak itself is very sharp. This might be an indicator of a short range order due to long-living pairs of mercury atoms, in which the atoms have a strong mutual correlation. Indeed, such dimers of the bound $\mathrm{Hg}^{*}$ atoms the reader can clearly distinguish in the respective snapshot in the 


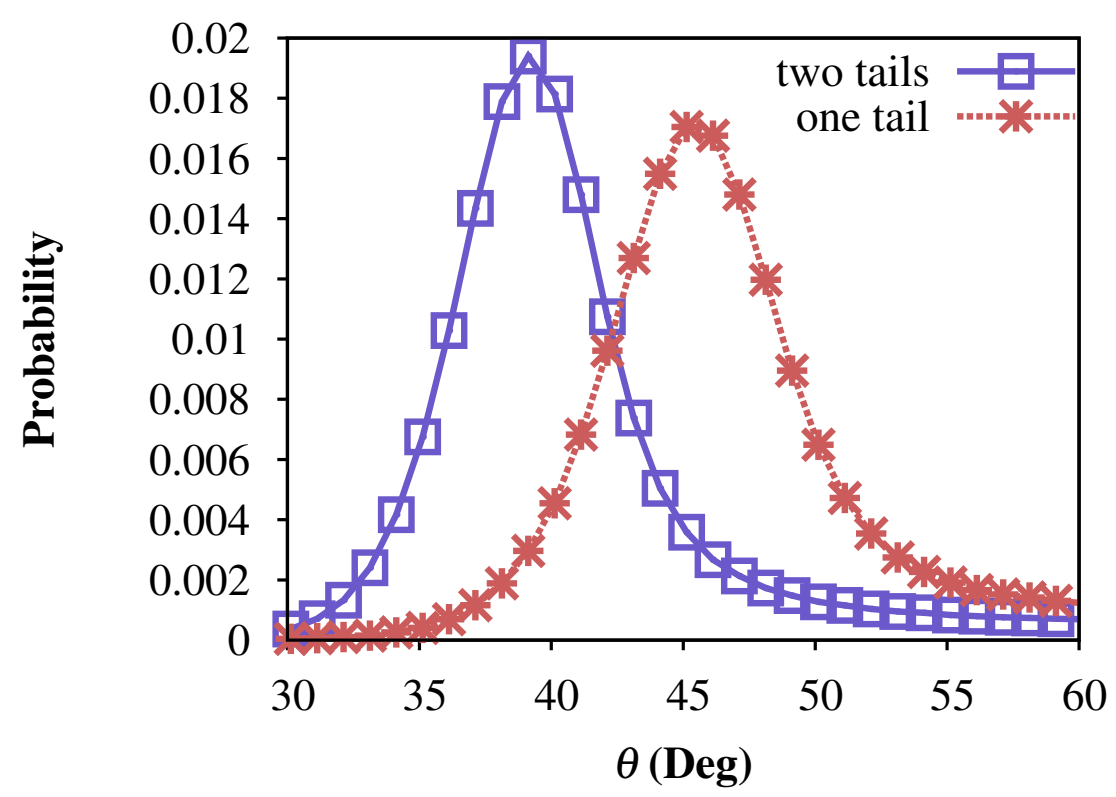

Figure 5.5: Comparison of the tilt angle distributions for the systems with single- and double-tailed surfactants. $\sigma=1.6114 \mathrm{~nm}^{-2}, A_{c s}=903.5 \mathrm{~nm}^{2}, T=293 \mathrm{~K}$.

laying-down as well as in the standing-up conformations (Fig. 5.6a). In order to draw a direct comparison of the headgroup packing of the single-tailed surfactants with the packing of the double-tailed ones it is useful to analyze the distribution of sulfurs, since there is an equal number of these species in both systems. From the snapshot of the sulfurs in the single-tailed system (Fig. 5.7a) one can clearly see that the sulfurs reveal a higher degree of translational ordering compared to the bound mercury in these system. Nevertheless, there is also a lot of grain boundaries between the ordered patches of sulfurs. The arrangement of the sulfur atoms in the ordered patches corresponds clearly to a 2D oblique lattice, which is very close to a hexagonal one. The position of the first peak in the radial distribution function of the sulfurs in the single-tailed surfactants coincides very well with the position of the second peak for the systems with the double-tailed surfactants (Fig. 5.7b). The sharp first peak in the radial distribution function of the sulfurs in the double-tailed surfactants is due to the $\mathrm{S}-\mathrm{Hg}-\mathrm{S}$ bond. Generally, the peaks of the radial distribution of sulfurs in the singled-tailed molecules are less pronounced compared to the double-tailed systems, indicating that the elimination of the $\mathrm{S}-\mathrm{Hg}-\mathrm{S}$ bond allows for larger fluctuations in the positions of the sulfur headgroups. 

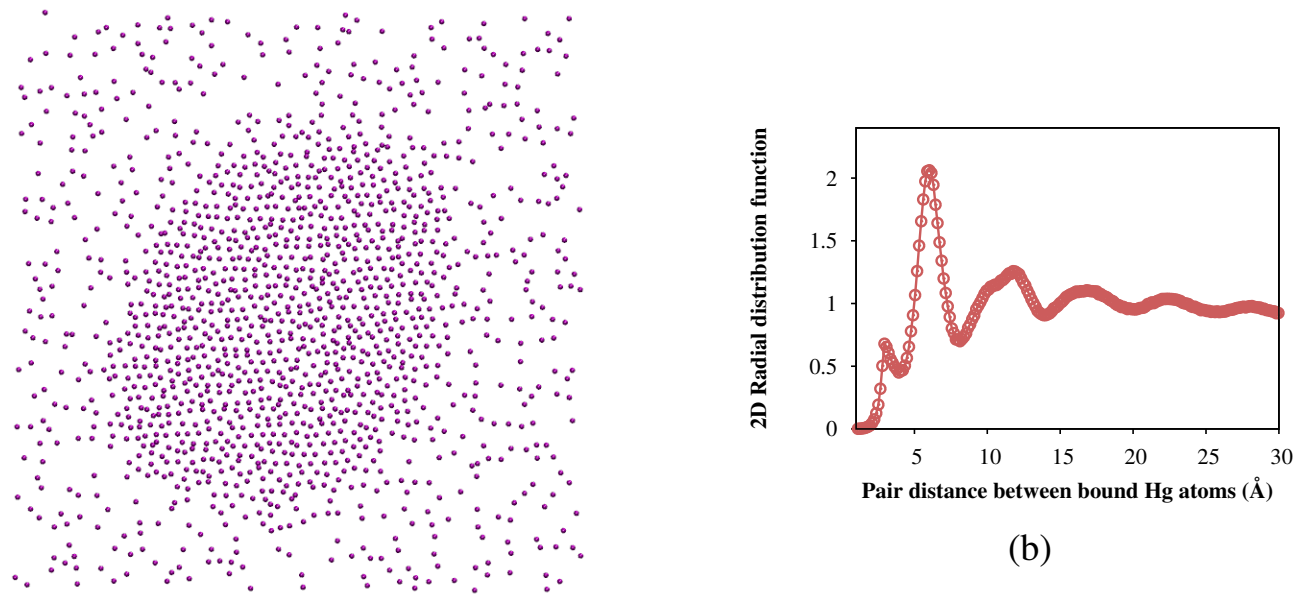

(b)

(a)

Figure 5.6: Snapshot of the bound mercury atoms (a) and the respective radial distribution function (b) for the system with single-tailed surfactants. $\sigma=1.6114 \mathrm{~nm}^{-2}$, $A_{c s}=903.5 \mathrm{~nm}^{2}, T=293 \mathrm{~K}$
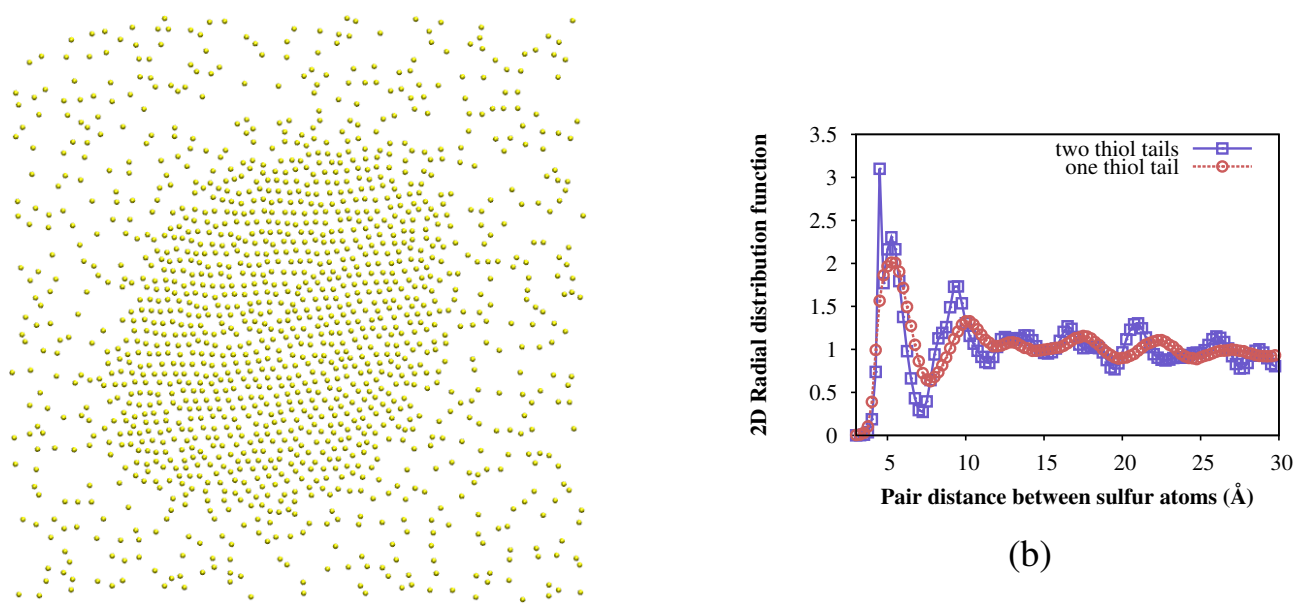

(b)

(a)

Figure 5.7: (a) Snapshot of sulfur headgroups for the system, in which a surfactant molecule consists of a single thiol attached to a single mercury atom. (b) Comparison of the sulfur radial distribution functions for the systems with single- and double-tailed surfactants. $\sigma=1.6114 \mathrm{~nm}^{-2}, A_{c s}=903.5 \mathrm{~nm}^{2}, T=293 \mathrm{~K}$. 


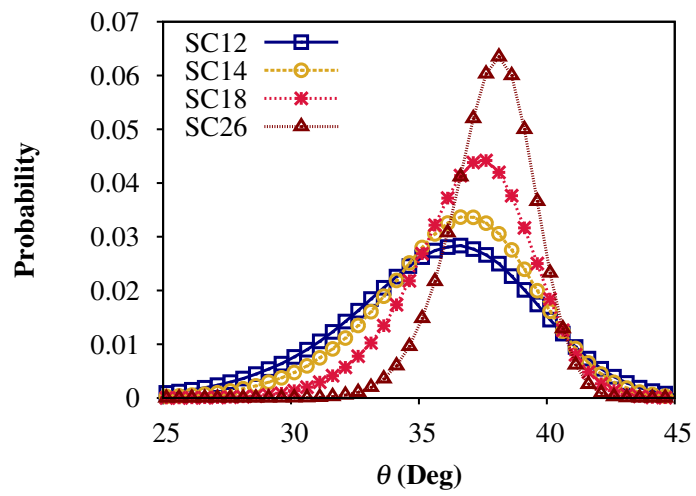

(a) Tilt angle distribution.

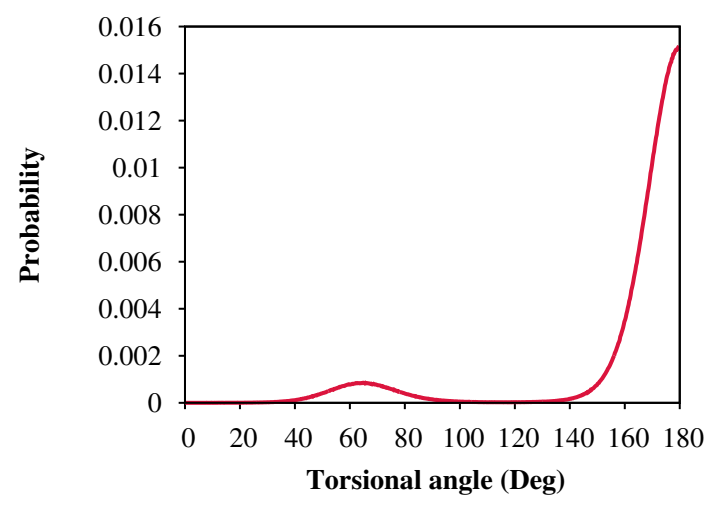

(c) Distribution of the $\mathrm{CH}_{2}-\mathrm{CH}_{2}-\mathrm{CH}_{2}-\mathrm{CH}_{3}$ torsional angles for the SC18 SAM.

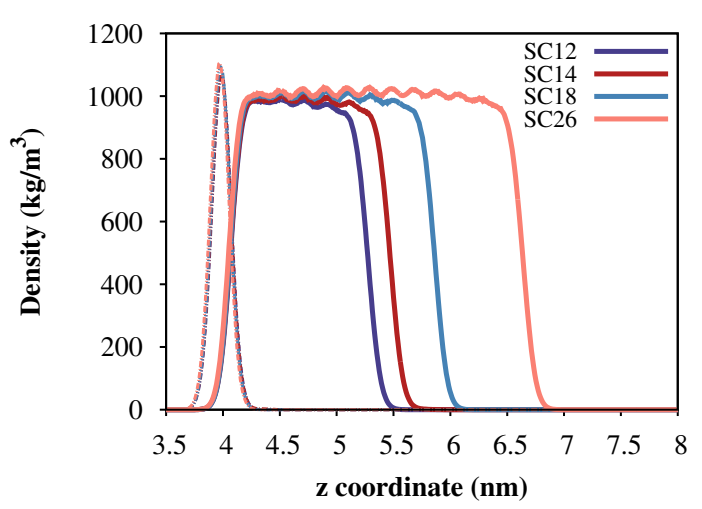

(e) Density profiles of alkyl tails (solid line) and sulfur headgroups (dashed line).

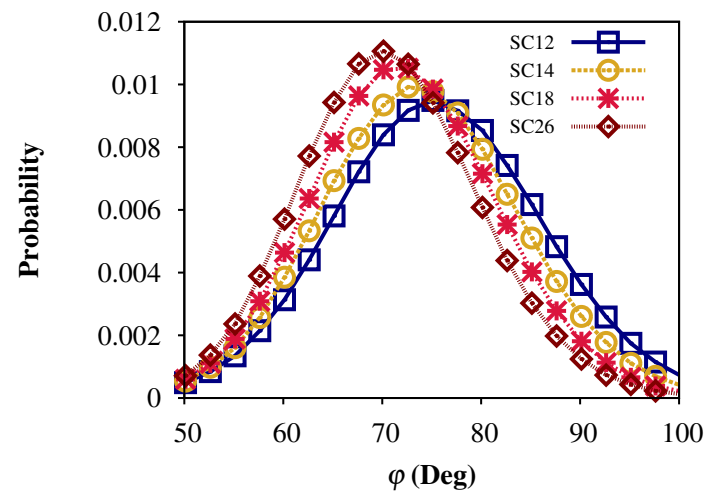

(b) Azimuthal tilt direction.

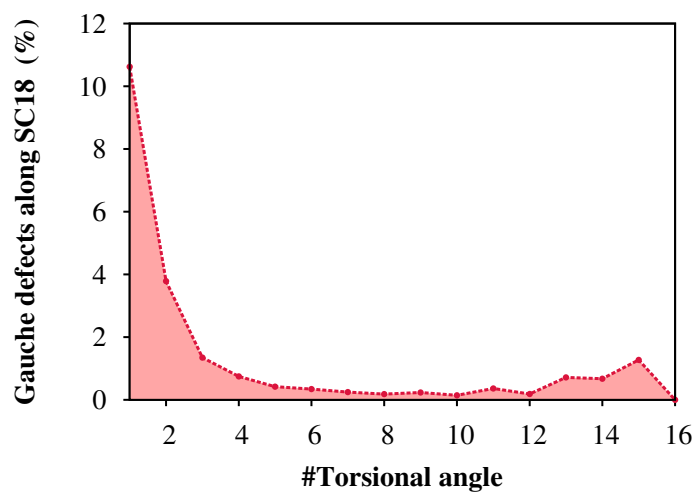

(d) Percentage of gauche defects per torsional angle along SC18.

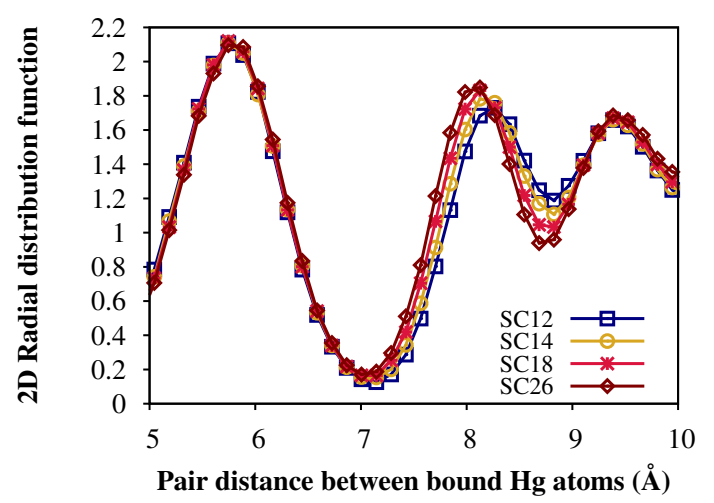

(f) 2D radial distribution function of bound mercury atoms.

Figure 5.8: Various packing characteristics of dodecanethiol (SC12), tetradecanethiol (SC14), octadecanethiol (SC18) and hexacosanethiol (SC26) SAMs on Hg. 


\subsection{Chain length effects}

In this section the dense self-assembled monolayer of dodecanethiol (SC12), tetradecanethiol (SC14), octadecanethiol (SC18) and hexacosanethiol (SC26) are studied by the simulations in the $N \mathcal{P} T$ ensemble. Fig. 5.8a shows distributions of the tilt angles for the thiols of various tail lengths, where one can see that the tilt angle slightly increases from $36.5^{\circ}$ to $38^{\circ}$ as the alkyl chain length growth from 12 to 26 carbons per tail. The tilt angle for SC18 thiols is $37.4^{\circ}$ accordingly. The collective azimuthal tilt direction of thiols decreases from $75^{\circ}$ to $70^{\circ}$ as the tail length growth (Fig. 5.8b). The distribution of the terminal $\mathrm{CH}_{2}-\mathrm{CH}_{2}-\mathrm{CH}_{2}-\mathrm{CH}_{3}$ torsional angles (for SC18) shows that in addition to the large peak at $180^{\circ}$ there is also a small, but very clear, peak at $\sim 63^{\circ}$. The peak at $180^{\circ}$ corresponds to the situation of neighboring bonds lying in the same plane and the peak at $\sim 63^{\circ}$ is an indicator that there is a number of gauche defects present in the alkylthiol conformations. The distribution of the gauche defects along the SC18 backbone, shown in Fig. 5.8d, supports this finding. For the calculation of this distribution all torsional angles between $30^{\circ}$ and $100^{\circ}$ were counted as gauche defects. The first torsional angle, $\mathrm{S}-\mathrm{CH}_{2}-\mathrm{CH}_{2}-\mathrm{CH}_{2}$, possesses on average the largest number of the gauche defects, most probably stemming from the mismatch between the planes, in which the torsions $\mathrm{CH}_{2}-\mathrm{S}-\mathrm{S}-\mathrm{CH}_{2}$ and $\mathrm{S}-\mathrm{CH}_{2}-\mathrm{CH}_{2}-\mathrm{CH}_{2}$ lie. This mismatch arises as consequence of the collective tilt angle. Then along the SC18 backbone the portion of the gauche conformations substantially decreases and rises again as one approaches the opposite end of the molecular chain. Such behavior is consistent with the generally higher amount of gauche defects at the ends of the alkyl chains [164]. It is also not difficult to estimate the influence of the gauche defects on the tilt angle, $\theta$, by computing $\theta$ exactly as was done in the experiments [64], according to which $\theta=180^{\circ} \arccos \left(d_{S A M} / l_{t h}\right) / \pi$ with $d_{S A M}$ being the width of the thiol SAM as obtained from the measurements and $l_{t h}$ is the length of the thiol molecule assuming it is in the alltrans conformation. According to the united-atom approach used in my thesis the length of the SC18 in all-trans conformation is $d s_{\|}+17 d c_{\|}=23.4 \AA$, where $d s_{\|}=1.53 \AA$ and $d c_{\|}=1.286 \AA$ are the projections of the $\mathrm{S}-\mathrm{C}$ and $\mathrm{C}-\mathrm{C}$ bonds on the molecular axis. Now the average SC18 SAM thickness obtained from the simulation is $18.32 \AA$. This yields $\theta=180^{\circ} \arccos (18.32 / 23.4) / \pi=38.5^{\circ}$. This is approximately $1^{\circ}$ larger than the respective MD value, where the influence of the gauche defects was naturally taken 


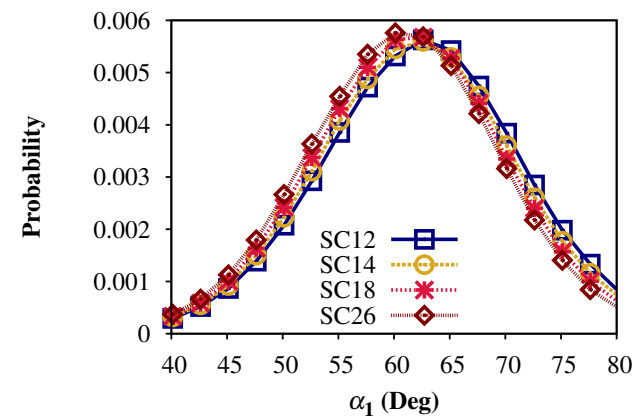

(a)



(c)

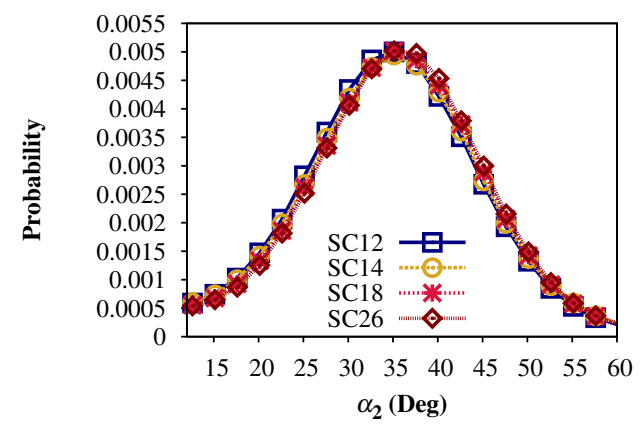

(b)

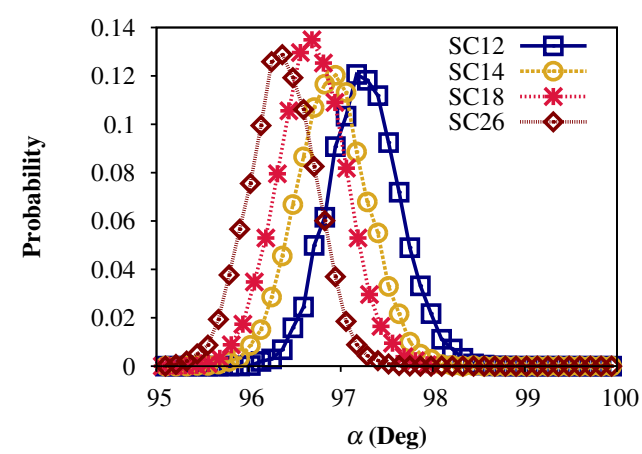

(d)

Figure 5.9: Angular distributions of the 1st (a), 2nd (b) and 3rd (c) nearest neighbors of the $\mathrm{Hg}^{*}$ atoms, and the distribution of the angle between the base vectors of the respective unit cell (d) for the monolayers of thiols of different tail lengths.

into account.

Fig. 5.8e demonstrates typical density profiles developed by the molecules of various lengths. Slight oscillations are due to the fact that the monolayers are crystalline. The typical density in the middle of the thiol SAM reaches about $1000 \mathrm{~kg} / \mathrm{m}^{3}$, which is higher compared to the typical liquid phases of alkanes (e.g. $777 \mathrm{~kg} / \mathrm{m}^{3}$ for C18). The 2D pair distribution function of the bound mercury atoms is given in Fig. 5.8f. From the figure one can a distinguish a small decrease in the second nearest neighbor distance with the increase in the tail length. This and the decrease in the angle between the unit cell base vectors of bound mercury (see below) amounts to a slight increase in the thiol surface coverage from 4.23 to $4.29 \mathrm{~nm}^{-2}$ for SC12 and SC26 SAMs, respectively. A similar slight increase in the molecular packing upon the increase of the molecular length was also observed for partially fluorinated alkylthiol monolayers on gold [165]. 
One sees only very small changes in the angular distributions of the 1st, 2nd and 3rd nearest neighbors for the bound mercury (Figs. 5.9a, 5.9b, 5.9c). This is why in order to capture the change of the unit cell upon increase in the chain length with a finer resolution the distributions of the angle, $\alpha$, between the base vectors is computed from the simulation box sizes, which fluctuate as the time proceeds in the simulations in the $N \mathcal{N} T$ ensemble. Fig. 5.9d shows these distributions. One can clearly see that the angle $\alpha$ decreases from about $97.3^{\circ}$ to $96.3^{\circ}$ as the chain length grows from SC12 to SC26, respectively.

\subsection{Pressure effects}

In this section the response of the SC18 SAM on liquid mercury upon uniform lateral compression is analyzed. Fig. 5.10a shows the 2D radial distribution function for bound mercury atoms. We can see that upon uniform lateral compression the peaks of the 1st and 2nd (less pronounced) nearest neighbors tends to shift to larger distances, but the peak for the 3 rd nearest neighbors seems to stay in place. This happens due to the change of the angle, $\alpha$, between the base vectors of the bound mercury unit cell. It is seen from Fig. 5.10b that this angle increases upon the compression. The respective distributions become lower and wider indicating the thiol SAM becomes more disordered as the lateral pressure growth. Since the 3rd nearest neighbor (3NN) peak in the radial distribution is not changing with pressure, one can conclude that the change of $\alpha$ proceeds through the shifts of bound mercury atoms along the $3 \mathrm{NN}$ direction. The highest lateral pressure, at which I managed to obtain the stable SC18 SAM, was -310 atm (the minus sign means that the pressure is applied in the direction of the center of the SAM from all the lateral sides of the simulation box). The pressure of -310 atm would correspond to the surface tension of about $628 \mathrm{mN} / \mathrm{m}$, which is more than $50 \%$ higher than the surface tension of mercury/air interface in the present computational model. Upon further increase in the lateral pressure the SC18 SAM becomes destabilized and eventually collapses, in the simulations this leads to the decrease of $\alpha$ until the simulation box becomes unstable and is destroyed. In contrast, Langmuir monolayers on water/oil interface typically collapse when the interfacial surface tension reaches the value of the water/oil interface without surfactants [166]. Such high stability of the SAM of SC18 surfactants on liquid mercury, compared to Langmuir films on water, is probably due 
to the chemisorption of thiol onto mercury and due to the high surface tension of the surface of liquid mercury itself.

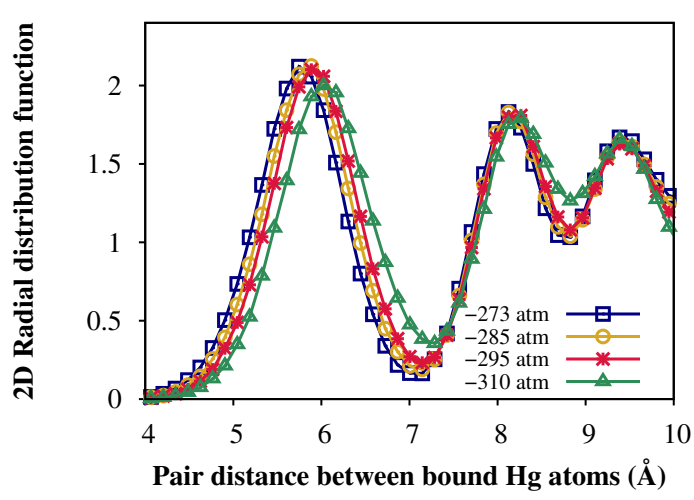

(a)



(b)

Figure 5.10: Pair distribution function (a) and the distribution of the angle between the base vectors of the unit cell of the bound mercury for various values of lateral pressure for the SC18 SAM.

\subsection{Effects of surface coverage in the dense SAM and influence of the interaction strength between bound mercury atoms}

Let us recall the force field model described in the beginning of Chap. 4. One of the unfixed interactions there was the lateral interaction between the bound mercury atoms. For simplicity and since bound mercury is no longer metallic, and, consequently, it cannot interact as strong as the bulk mercury with itself, the interaction for the bound mercury was taken to be the same as for the alkyl tails with the bulk mercury. As a reminder, this interaction was of the double-exponent form given by Eq. 4.10, with the parameters $A_{1}=0.5 \mathrm{kcal} / \mathrm{mol}$ and $b=2.0 \AA^{-2} . A_{1}$ was the interaction strength and $b$ controlled the attraction range. In this section the strength of the interaction between the bound mercury atoms is varied in order to better understand the influence of this parameter on the thiol SAMs on liquid mercury. Physically this will also allow to acquire insights into the possible structural transformations within the SAM for various surface 


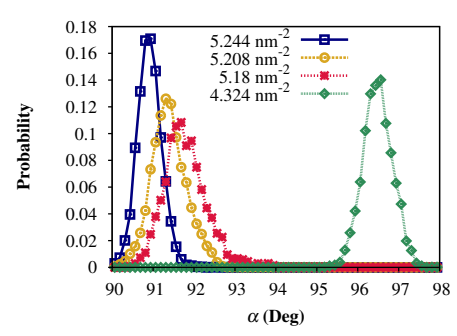

(a)

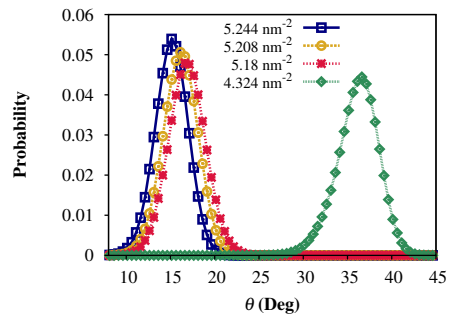

(b)

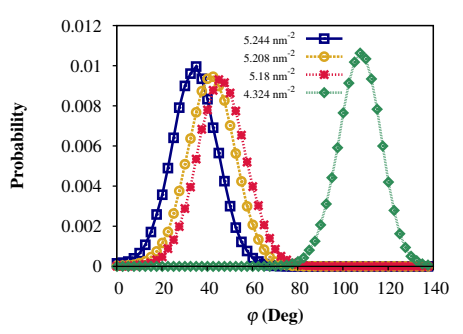

(c)

Figure 5.11: Distributions of the angle between the base vectors of the bound mercury unit cell (a), tilt angle (b) and azimuthal tilt direction (c) for $\sigma=5.244,5.208,5.18$, and $4.324 \mathrm{~nm}^{-2}$, which correspond to $A_{1}=2.233335535,1.45,1.228$ and $1.139 \mathrm{kcal} / \mathrm{mol}$.

coverages, $\sigma$, of alkylthiols in the regime of a very dense molecular packing. The simulations were performed for the SC18 molecules and the parameter $b$ was fixed at the value of $0.891 \AA^{-2}$ as in the case of the bulk mercury. The parameter $A_{1}$ took the values of $2.233335535,1.45,1.228$ and $1.139 \mathrm{kcal} / \mathrm{mol}$, which yielded 5.244, 5.208, 5.18, and $4.324 \mathrm{~nm}^{-2}$ for the equilibrium SC18 surface coverage as obtained from the simulations as $\left\langle N_{t h} / A_{c s}\right\rangle$, respectively. Here, as before, $N_{t h}$ is the number of thiols per side of the $\mathrm{Hg}$ film and $A_{c s}$ is the cross-section of the simulation slab at a given time instance. The upper boundary for the parameter $A_{1}$ (as well as the value of $b$ ) is taken from the optimized density-independent (ODI) force field for the simulation of the surface of liquid mercury derived in Chap. 3. The values of $A_{1}$ below the lower boundary of $1.139 \mathrm{kcal} / \mathrm{mol}$ do not virtually influence the structure of the monolayer according to my simulations. For the surface coverage, $\sigma$, in the range between 5.244 and $5.18 \mathrm{~nm}^{-2}$ the length of the base vectors remain basically unaltered (4.5 and 8.2 $\AA$ as obtained from the 1 st and 2nd peaks of the $\mathrm{Hg}^{*}$ pair distribution function) and the angle, $\alpha$, between the base vectors of the oblique unit cell changes very slowly from about $90.9^{\circ}$ to $91.8^{\circ}$, and as the surface coverage decreases further the angle $\alpha$ changes to $96.5^{\circ}$ (Fig. 5.11a). Similar behavior is also found in the distributions of the tilt angle, $\theta$, and the azimuthal tilt direction given by the angle $\phi$ (the angle between the $\mathrm{S}-\mathrm{Hg}-\mathrm{S}$ bond and the $x y$-projection of the molecular weight, recall Fig. 4.15). For $\sigma$ between 5.244 and $5.18 \mathrm{~nm}^{-2}$ the tilt angle varies very mildly from $15.1^{\circ}$ to $16.9^{\circ}$, following which it changes up to $36.6^{\circ}$ for $\sigma=4.324 \mathrm{~nm}^{-2}$ (Fig. 5.11b). Likewise, the average azimuthal tilt direction changes first slowly from $36.6^{\circ}$ to $46.4^{\circ}$ as $\sigma$ drops from 5.244 to $5.18 \mathrm{~nm}^{-2}$, and afterwards 
it takes on the value of $108^{\circ}$ for $\sigma=4.324 \mathrm{~nm}^{-2}$ (Fig. 5.11c). The tilt directions for $\sigma=5.244 \mathrm{~nm}^{-2}$ is close to the 2nd nearest neighbor direction $\alpha_{2}=30^{\circ}$ and the last azimuthal angle for $\sigma=4.324 \mathrm{~nm}^{-2}$ is close to one of the equivalent 1 st nearest neighbor ( $1 \mathrm{NN})$ directions given by the angle $\alpha_{1}$ as determined from the respective angular distributions of the nearest neighbors. The 1 st $1 \mathrm{NN}$ direction is at about $\alpha_{1}=60^{\circ}$ and the 2 nd is located at $\alpha_{1}=\pi-60^{\circ}$ (two $\mathrm{Hg}^{*}$ atoms in the 1 st coordination shell) 1 If one decreases the interaction strength between the bound mercury atoms, $A_{1}$, further (and respectively the thiol lateral packing) the system spontaneously undergoes a transition to the state with a higher tilt angle as is shown in Fig. 5.12 for $A_{1}=1.1502 \mathrm{kcal} / \mathrm{mol}$.

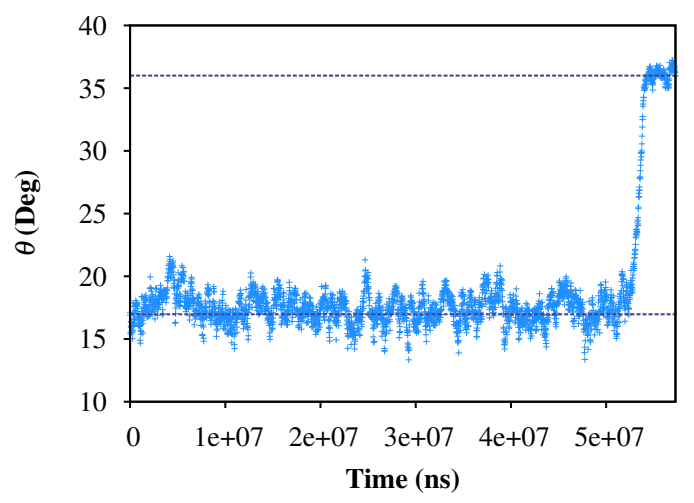

Figure 5.12: Time evolution of the tilt angle for $A_{1}=1.1502 \mathrm{kcal} / \mathrm{mol}$. One can see that the system spontaneously "jumps" into a state with a higher tilt angle $\theta=36^{\circ}$ after staying about $50 \mathrm{~ns}$ in the state with $\theta=16^{\circ}$, in which the surface coverage of the system corresponded to $5.172 \mathrm{~nm}^{-2}$. Such abrupt transformation from one state to another is an indicator of a first-order phase transition.

It is also interesting to mention that at the higher packing of the dense SAM of standing thiols the amount of gauche conformations in the vicinity of the sulfur headgroups substantially decreases (Fig: 5.13), such that, for example, the percentage of the gauche defects in the first torsional angle $\left(\mathrm{S}-\mathrm{CH}_{2}-\mathrm{CH}_{2}-\mathrm{CH}_{2}\right)$ drops from $10.3 \%$ down to $3.7 \%$ as $\sigma$ rises from 4.324 to $5.244 \mathrm{~nm}^{-2}$. Finally, I have to note that the results obtained in this section for $A_{1}=1.139 \mathrm{kcal} / \mathrm{mol}$ and $b=0.891 \AA^{-2}$ are qualitatively and quantitatively very similar to the ones for $A_{1}=0.5 \mathrm{kcal} / \mathrm{mol}$ and $b=2.0 \AA^{-2}$ used through out

\footnotetext{
${ }^{1}$ The $2 \mathrm{nd} \mathrm{Hg}^{*}$ atom should be actually located at $\alpha_{1}=\pi+60^{\circ}$, but all $\alpha_{1}>\pi$ are reflected to the upper hemisphere $\left(\alpha_{1} \leq \pi\right)$, since the arccos used in the calculations (recall Eq. 4.25 ) is defined in $[0, \pi]$. This means that the 2nd $1 \mathrm{NN}$ neighbor will be shown as the peak at $\alpha_{1}=\pi-60^{\circ}$ (rather than at $\alpha_{1}=\pi+60^{\circ}$ ) in the respective angular distribution.
} 


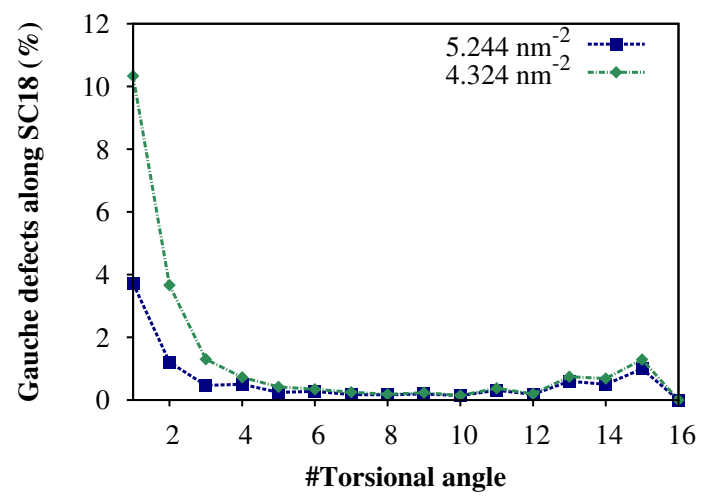

Figure 5.13: Percentage of gauche defects along the backbone of SC18 thiol at the surface coverage of 5.244 and $4.324 \mathrm{~nm}^{-2}$, which correspond to the strength of interaction, $A_{1}$ between bound mercury atoms of 2.233335535 and $1.139 \mathrm{kcal} / \mathrm{mol}$, respectively.

the thesis for the $\mathrm{Hg}^{*}-\mathrm{Hg}^{*}$ interaction. This basically means that the structure of thiol SAMs on $\mathrm{Hg}$ is not subjected to substantial changes for the bound mercury interaction parameters $A_{1} \in[0.5,1.139] \mathrm{kcal} / \mathrm{mol}$ and $b \in[0.891,2.0] \AA^{-2}$.

\subsection{Conclusions and Discussions}

The focus of this chapter was devoted to the dense crystalline SAM of octadecanethiols on the surface of liquid mercury. More precisely, the influence of such factors as temperature, molecular morphology, alkyl tail length and lateral pressure, as well as the strength of the $\mathrm{Hg}^{*}-\mathrm{Hg}^{*}$ interaction on the SAM structure was investigated. It is found that the monolayer preserves well its crystalline structure up to $T=330 \mathrm{~K}$. Afterwards, at $T=350 \mathrm{~K}$ the crystal structure in the monolayer melts away and it transforms into an amorphous film with the sulfur headgroups staying at the surface of mercury. For comparison, alkylthiol SAMs on Au preserve a crystalline order (though quite disturbed) at least up to $T=373 \mathrm{~K}$ [150]. Most probably the lack of the long-range order in the mercury substrate promotes the degradation of the crystalline order in the SAM on $\mathrm{Hg}$ at lower temperatures compared to Au. Moreover, the simulation with an alternative molecular architecture (when one thiol is attached only to a one $\mathrm{Hg}$ atom) reveals higher disorder in such systems and too large tilt angles compared to the case when a one $\mathrm{Hg}$ can chemically bound to two thiol molecules. The simulation in the $N \mathcal{P} T$ ensemble of 
the crystalline thiol monolayers periodic in all lateral directions allows to avoid the effect of finite boundaries, which was present for the crystalline islands of the standing-up thiols in the previous chapter. No qualitatively different structural transformations are found for the studied lengths (from 12 to 26 carbons) of the alkyl tails. The stable SC18 monolayer are obtained up to the value of the lateral pressure of $-310 \mathrm{~atm}$. Upon lateral compression the monolayer relaxes via shearing of the headgroups along the $3 \mathrm{NN}$ direction by a finite displacement, which coincides with the collective direction of the $\mathrm{S}-\mathrm{Hg}-\mathrm{S}$ bond. Shearing relaxation as the response to the lateral pressure is a well-know mechanism of relaxation in the case of lipid monolayers on a water/oil interface [167]. As shown above, changing the strength of the $\mathrm{Hg}^{*}-\mathrm{Hg}^{*}$ interaction appears to be a convenient way of a fine control of the thiol surface coverage within a crystalline densely packed monolayer of thiols on $\mathrm{Hg}$ in a computer simulation. Using this approach it is demonstrated that upon increase in the SC18 surface packing one should expect a weak first order transition in the azimuthal tilt from approximately the $1 \mathrm{NN}$ direction to the 2NN direction. This transition involves complex changes in the tilt angle as well as in the unit cell of the headgroups. A similar first order transition from tilted to non-tilted thiols on liquid mercury upon the increase in the surface coverage was also proposed previously [65]. Finally, the tilt angle $\theta=37.4^{\circ}$ obtained in this chapter for the periodic crystalline SAM of SC18 thiols is smaller than $39^{\circ}$ obtained for the respective crystalline island in Chap. 4 but it is still larger than $27^{\circ}$ obtained by X-Ray experiments [64]. As discussed in the introduction, sharp tilt angles $\left(\leq 30^{\circ}\right)$ of the amphiphile surfactants on water may be explained by the strong hydrophobic interaction of the tails with water. On the other hand, sharp $\left(\sim 30^{\circ}\right)$ tilt angles on gold can be rationalized by the strong directional chemical binding of the sulfur headgroups to "frozen" Au atoms, whereas on the surface of liquid $\mathrm{Hg}$ the bound mercury is highly mobile. In addition, thiols have an attractive interaction with the mercury substrate. Consequently, there is no straightforward reason to anticipate that the tilt angle of thiols on liquid $\mathrm{Hg}$ should be similar to the ones of thiols on gold or of amphiphiles on water. More structural data on the alkylthiol SAMs on $\mathrm{Hg}$ by other experimental techniques is required in order to clarify the value of the tilt angle and whether the headgroup packing is rectangular or rather (weakly) oblique. 


\section{6 | Final remarks and outlook}

Today self-assembled alkylthiol monolayers on liquid metals are routinely used in organic electronics to study charge transport through hybrid metal-SAM-metal or semiconductor-SAM-metal junctions [50]. The properties of such junctions are tailored by varying the form of the mercury drop-electrode with a grafted SAM on it [51]. This inevitably leads to structural transformations within the SAM, meaning that the junction properties are closely related to the underlying structure of the SAM. The molecularlevel understanding of the factors that define these structural transformations is crucial for a better design of the metal-SAM-metal junctions.

Despite of the popularity of the alkylthiol SAMs on liquid metals in organic electronics the knowledge of the molecular structure of these systems is limited and chiefly originates from X-Ray studies [61, 62, 64, 65] yielding not always consistent reports. The structure and resulting properties of a thiol SAM may strongly depend on the way the SAM is prepared and external conditions [6-9]. Therefore, more experimental data obtained by other experimental techniques under different conditions is vitally needed in order to gain more complete picture of the molecular-scale self-assembly on liquid metal substrates. The optical tensiometry experiments [27] (covered in detail in Chap. 1) provide useful insights into the kinetics of the alkylthiol SAM formation on liquid mercury, but supply no microscopic structural data.

As alternative to experiments, in this work I undertake a different approach, namely, a large-scale molecular simulation, in order to investigate the alkylthiol self-assembly on liquid mercury. Because of the high complexity of the systems under consideration and a multitude of intricate intermolecular interactions, no computational study of these systems has been previously performed. Specifically, simulation studies face the following significant challenges: 
- liquid mercury, though "merely" used as a substrate in the simulations, has to be treated fully atomistically and allowed to evolve according to dynamic equation of motion in a molecular simulation in order to ensure that the SAM relaxation on liquid mercury includes the molecular features of the thiol binding to mercury, as well as the liquid nature of the substrate;

- the lack of experimental data, such as adsorption energies of alkylthiols on liquid mercury, which would allow to parametrize the interaction between thiols and the Hg substrate;

- parametrization of the above interactions using ab-initio techniques is highly nontrivial, since it requires the sampling of the interaction of an alkylthiol molecule with a cluster of at least $100 \mathrm{Hg}$ atoms in order to take into account the metallic nature of mercury;

- no appropriate force field for liquid mercury exists, which is suitable even for the large-scale simulations of the bare surface of liquid mercury;

- extremely long relaxation times ${ }^{1}$ of thiol SAMs on liquid Hg (recall Sec. 1.2, which are basically unattainable for standard molecular simulations;

- severe finite-size effects, because of which one has to simulate exceedingly large systems (as for an atomistic or semi-atomistic computer simulation) in order to be able to accurately localize regions of phase coexistence.

State-of-the-art Molecular Dynamics (MD) simulation techniques are used throughout the dissertation for the simulations of the thiol self-assembly on liquid mercury, because this method is able to give an accurate information on the molecularscale. In Chap. 3 a method for computationally efficient simulation of a bare surface of liquid mercury at room temperatures is derived. For this purpose the latest and most successful atomistic force fields of liquid mercury are investigated and compared. Namely, the following atomistic models of liquid $\mathrm{Hg}$ are used: (i) the Raabe model [119, 120] and (ii) the density-independent (DI) double-exponent model [121, 122], as well as such

\footnotetext{
${ }^{1}$ simulation times attained in the present thesis reach $\sim 0.1-0.4 \mu \mathrm{s}$, whereas the experimental time scales of SAM formation reach out to several hours and more.
} 
models as (iii) the density-dependent (DD) double exponent model [126], and two recent embedded-atom models, (iv) the EAM2013 [131] and (v) EAM2006 [133] models. Characteristic features of the $\mathrm{Hg}$ surface at room temperatures are its extremely dense, but liquid, structure and a high surface tension. With this perspective the Raabe and the DD models are discarded from the treatment, because the former model yields a crystallized mercury film at the room temperature, and using the free-energy arguments I show that the latter model results in the wrong phase behavior of liquid $\mathrm{Hg}$ at room temperatures caused by the unphysical density-dependence of one of the coefficients. The analogical analysis of the other three models (DI, EAM2013 and EAM2006) has not revealed such spurious effects. The DI model yields the correct behavior of liquid mercury but a too low surface tension, whereas the EAM models agree much better with experimental data. Since the EAM models are generally more computationally demanding (because of the density dependence) compared to the DI model, the latter model was optimized in order to improve the agreement with the experimental value. Consequently the optimized density-independent (ODI) force field is used in Chaps. 4 and 5 for the simulations of alkylthiol SAMs on liquid $\mathrm{Hg}$. The optimization procedure of the DI model is outlined in detail in Sec. 3.2. Moreover, it is also shown in Sec. 3.2 that the ODI force field yields a quite good description for the bulk of mercury at the temperature region of practical interest.

In Chap. 4 the self-assembly of alkylthiols as the function of their surface coverage on liquid $\mathrm{Hg}$ is investigated. In Sec. 4.1.1 the molecular model of the thiol surfactants on liquid mercury is formulated step-by-step. Substantial time savings are achieved by using (i) highly parallelized large-scale MD simulations [77], (ii) multiple time-step algorithm outlined in Chap. 2, and due to (iii) the usage of the united-atom representation of the surfactants (recall Fig. 1.7). Two coexistence regions are observed in my simulations, namely, (i) the coexistence of a 2D phase of the laying-down thiols with a very dilute 2D gas (basically bare $\mathrm{Hg}$ surface) on liquid mercury, and (ii) the coexistence of the laying-down molecules with islands of the standing-up thiols featuring long-range order. These findings are consistent with available experimental data [64, 65]. My MD simulations reveal, that the 2D conformations of the laying-down molecules represent liquid aggregates of the laying-down molecules growing up to the full coverage of the laying-down thiols rather than a 2D gas of laying-down thiols suggested previously [64, 65]. It is shown that the thiol headgroups pack into a $2 \mathrm{D}$ weakly oblique lattice 
with the angle, $\alpha \sim 97^{\circ}$, between the base vectors and having two alkylthiol molecules per unit cell. The dimensions of the obtained unit cell are very close to the experimental ones for $T=299 \mathrm{~K}$ [64], with a slight difference that $\alpha=90^{\circ}$ was suggested therein [64].

In Chap. 5 the influence of such factors as temperature, molecular morphology, surfactant chain length, lateral compression and of elevated surfactant surface coverage in the dense crystalline phase on the thiol SAMs on liquid mercury is analyzed. It is found that the crystalline island of octadecanethiols (obtained in Chap. 4 at $T=293 \mathrm{~K}$ ) loses its long-range order and "melts" already at $T=350 \mathrm{~K}$. The simulation of the alkylthiol SAM with "single-tailed surfactants" (i.e. without the $\mathrm{S}-\mathrm{Hg}-\mathrm{S}$ bond as shown in Fig. 1.7b) also yields a crystalline phase of standing-up thiols, which is characterized by larger degree of disorder and higher tilt angles compared to the thiol surfactants with the $\mathrm{S}-\mathrm{Hg}-\mathrm{S}$ bond. Giving this and the fact that in the majority of organomercuric compounds mercury has a valency of two, I consider such surfactant architecture rather unrealistic. Simulations with constant stress allowed to obtain structural characteristics of the dense thiol SAMs on liquid mercury at high surface coverage, which were free of the effects due to the finite size of the crystalline phases in the simulation. The observed structures featured no significant differences with the variation of the tail length as well as the structure of the finite thiolate islands from Chap. 4. The lateral compression simulations indicated that upon the increase of the magnitude of the lateral pressure the degree of disorder grows and the monolayer is destabilized by the shift of the headgroups along the third-nearest-neighbor direction. Finally, the simulations at higher thiol surface coverage (compared to the one in the islands of the standing-up thiols) display a first-order tilting transition characterized by a sharp decrease of the collective tilt angle with the increase in the surfactant surface coverage. The results obtained in Chap. 5 were compared with experiments where possible.

The present work sets the ground for future theoretical studies of the self-assembly of organic films on liquid metals, which provide a very rich and intricate phase behavior. As was briefly mentioned in Chap. 1, in organic electronics hybrid metal-SAM-metal junctions typically consist of one crystalline and one liquid metal electrode opposed to each other [50]. Different packings of thiol SAMs on mercury and on gold electrodes can, in principle, result in defects at the interfaces, where two SAMs from the opposing electrodes are brought into contact. Since the SAM on the liquid metal electrode is 
laterally much more mobile compared to the one on the crystalline electrode, one could intuitively expect that the SAM on liquid mercury could rearrange itself in order to minimize the interfacial free energy, and in such a way adopt the structure of the SAM on the opposite electrode. This possibility may depend, for instance, on the length deference of thiols on the opposing SAMs. An extension of the simulations presented in the current dissertation to model, for example, $\mathrm{Au}-\mathrm{SAM}-\mathrm{Hg}$ junctions will help to elucidate what happens on a molecular level with both SAMs on Au and Hg. Alternatively, it is interesting to model the response of the $\mathrm{Hg}-\mathrm{SAM}-\mathrm{Au}$ junction under external stress conditions, because the junction properties are typically adjusted by pressing the mercury drop-electrode against the opposite one. Apart from mercury, another liquid metals (e.g. alloys of gallium and indium) are used to produce drop-electrodes [50], and the simulations of SAMs on different liquid metal substrates represents an interesting and challenging extension of the modelling approach presented in my dissertation. Moreover the extension of the current work to bioorganic surfactants, which were intensively studied on crystalline supports [168], is an appealing task as well. Finally, the force field and simulation strategies developed here can help to understand the formation mechanism and structure of the sheet-like monocrystalites of alkylthiols attached to a mercury backbone found in the sonication experiments [115]. 


\section{A | Lists of systems simulated in Chap. 4}

\begin{tabular}{|c|c|c|c|c|}
\hline$N_{m}$ & $\sigma\left(\mathrm{nm}^{-2}\right)$ & $t_{e q}(\mathrm{~ns})$ & $t_{s}(\mathrm{~ns})$ & Final configuration \\
\hline 27 & 0.53788 & 25.676 & 89.504 & PF of 1st LD thiol layer \\
\hline 40 & 0.79687 & 3.018 & 91.982 & near FF of 1st LD layer \\
\hline 56 & 1.1156 & 35.928 & 202.072 & $\begin{array}{l}\text { oversaturated 1st LD layer, } \\
\text { distinct standing thiols, } \\
\text { no clear SU thiol island formation }\end{array}$ \\
\hline 64 & 1.27498 & 279.500 & 0.000 & $\begin{array}{l}\text { top: oversaturated 1st LD layer, } \\
\text { bottom: crystalline (cr.) SU island }\end{array}$ \\
\hline 72 & 1.43436 & 133.200 & 145.800 & cr. SU islands from both sides \\
\hline 72 & 1.43436 & 339.950 & 0.000 & $\begin{array}{l}\text { top: oversaturated 1st LD layer, } \\
\text { bottom: cr. SU thiol island }\end{array}$ \\
\hline 80 & 1.59373 & 225.936 & 118.514 & $\begin{array}{l}\text { cr. SU islands from both sides, } \\
\text { traces of ordered arrangement } \\
\text { of headgroups in cr. SU islands }\end{array}$ \\
\hline 80 & 1.59373 & 120.000 & 134.900 & cr. SU islands from both sides \\
\hline 90 & 1.79295 & 100.000 & 103.300 & cr. SU islands from both sides \\
\hline 104 & 2.07185 & 120.000 & 174.920 & $\begin{array}{l}\text { top: metastable spiral SU island } \\
\text { bottom: cr. SU island }\end{array}$ \\
\hline 144 & 2.86872 & 40.000 & 82.000 & cr. stripes of SU thiols from both s \\
\hline
\end{tabular}

Table A.1: Octadecanethiol (SC18) systems for the overall system cross-section of $9.936 \times 10.104=100.39 \mathrm{~nm}^{2} . N_{H g}=31852$. Notations: $N_{m}$ is the number of surfactant molecules per side of the $\mathrm{Hg}$ film, overall number of surfactants (R-S-Hg-S-R), $N_{m}^{t o t}$, is $2 N_{m}$; the number of thiol molecules, $N_{t h}$, per side of the $\mathrm{Hg}$ film is $2 N_{m} ; \sigma=N_{t h} /\left(L_{x} L_{y}\right)$ is the thiol surface coverage; $t_{e q}$ is the equilibration time, $t_{s}$ is the sampling time, and the total simulation time is $t_{t o t}=t_{e q}+t_{s}$; PF and FF stand for the "partial" and "full" coverage, respectively; LD and SU mean the "laying-down" and "standing-up" thiol conformations, respectively. 


\begin{tabular}{l|l|r|r|l}
$N_{m}$ & $\sigma\left(\mathrm{nm}^{-2}\right)$ & $t_{e q}(\mathrm{~ns})$ & $t_{s}(\mathrm{~ns})$ & Final configuration \\
\hline \hline 27 & 0.53788 & 0.400 & 105.600 & PF of 1st laying-down (LD) thiol layer \\
\hline 40 & 0.79687 & 0.302 & 109.698 & PF of 1st LD thiol layer \\
\hline 56 & 1.1156 & 0.800 & 107.200 & FF of 1st LD thiol layer \\
\hline 72 & 1.43436 & 0.542 & 258.358 & $\begin{array}{l}\text { oversaturated 1st LD layer, } \\
\text { couple of standing upright (SU) thiols, } \\
\text { no clear island formation, }\end{array}$ \\
\hline 80 & 1.5937 & 1.800 & 307.250 & $\begin{array}{l}\text { small strongly disordered patches } \\
\text { of standing thiols, } \\
\text { no crystalline (cr.) SU thiol islands }\end{array}$ \\
\hline 104 & 2.07185 & 5.000 & 363.000 & $\begin{array}{l}\text { strongly disordered patches of SU thiols, } \\
\text { no cr. SU thiol islands }\end{array}$ \\
\hline 144 & 2.86872 & 118.000 & 172.150 & \begin{tabular}{l} 
cr. stripes of SU thiols from both sides \\
\hline
\end{tabular}
\end{tabular}

Table A.2: Dodecanethiol (SC12) systems for the overall system cross-section of $9.936 \times 10.104=100.39 \mathrm{~nm}^{2} . N_{H g}=31852$. Notations follow the ones in Tab. A.1.

\begin{tabular}{l|l|r|r|l}
$N_{m}$ & $\sigma\left(\mathrm{nm}^{-2}\right)$ & $t_{e q}(\mathrm{~ns})$ & $t_{s}(\mathrm{~ns})$ & Final configuration \\
\hline \hline 90 & 0.79687 & 3.00 & 29.30 & slightly oversaturated FF of 1st LD layer \\
\hline 126 & 1.1156 & 12.20 & 205.90 & oversaturated 1st LD layer \\
\hline 162 & 1.43436 & 104.00 & 97.59 & cr. SU islands from both sides \\
\hline 182 & 1.61144 & 128.00 & 95.70 & cr. SU islands from both sides \\
\hline 234 & 2.07185 & 124.00 & 79.75 & $\begin{array}{l}\text { top: cr. SU island, bottom: cr. SU stripe } \\
\text { spanning through system boundaries, } \\
\text { grain boundary in cr. SU thiol stripe between } \\
\text { domains of different collective tilt angles }\end{array}$
\end{tabular}

Table A.3: Octadecanethiol (SC18) systems for the overall system cross-section of $14.904 \times 15.156=225.9 \mathrm{~nm}^{2} . N_{H g}=73456$. Notations follow the ones in Tab. A.1. 


\begin{tabular}{r|l|r|r|r|l}
$N_{m}$ & $\sigma\left(\mathrm{nm}^{-2}\right)$ & $t_{e q}(\mathrm{~ns})$ & $t_{s}(\mathrm{~ns})$ & $\begin{array}{l}\text { Initial } \\
\text { config. }\end{array}$ & $\begin{array}{l}\text { Final } \\
\text { configurations }\end{array}$ \\
\hline \hline 504 & 1.1156 & 128.45 & 0.000 & $\mathrm{U}$ & $\begin{array}{l}\text { top: oversaturated 1st LD layer, } \\
\text { bottom: cr. SU island }\end{array}$ \\
\hline 650 & 1.43879 & 100.00 & 23.850 & $\mathrm{U}$ & $\begin{array}{l}\text { top: grain boundary between two } \\
\text { merged islands (cryst. and spiral), } \\
\text { bottom: two "frozen" separate } \\
\text { islands (one cryst. and one spiral) }\end{array}$ \\
\hline \hline 0 & 0.0 & 1.00 & 6.214 & & equilibrated bare Hg surface \\
\hline 120 & 0.26562 & 2.40 & 104.600 & $\mathrm{U}$ & PF of 1st LD thiol layer \\
\hline 240 & 0.53124 & 4.00 & 103.000 & $\mathrm{U}$ & PF of 1st LD thiol layer \\
\hline 378 & 0.83671 & 4.00 & 103.000 & $\mathrm{U}$ & slightly oversaturated 1st LD layer \\
\hline 504 & 1.1156 & 20.00 & 87.000 & $\mathrm{P}$ & cr. SU islands from both sides \\
\hline 650 & 1.43879 & 18.00 & 89.000 & $\mathrm{P}$ & cr. SU islands from both sides \\
\hline 728 & 1.61144 & 14.66 & 92.340 & $\mathrm{P}$ & cr. SU islands from both sides \\
\hline 953 & 2.10948 & 16.00 & 91.000 & $\mathrm{P}$ & cr. SU thiol stripes from both sides \\
\hline
\end{tabular}

Table A.4: Octadecanethiol (SC18) systems for the overall system cross-section of $29.808 \times 30.312=903.5 \mathrm{~nm}^{2} . N_{H g}=285670$. U stands for initial configuration of surfactant molecules placed with their alkyl tails upright on simple square lattice with lattice spacings $L_{x} / N_{x}$ and $L_{y} / N_{y}$ in x- and y-directions, respectively. $N_{x}$ and $N_{y}$ are the numbers of the surfactant molecules in $\mathrm{x}$ - and y-directions, respectively. $N_{x}$ and $N_{y}$ were chosen such that the initial coverage of surfactants was maximally uniform. $\mathrm{P}$ stands for initially preset crystalline islands of standing-up thiols with their tails upright. We observed ordering of the headgroups for all $\mathrm{P}$-initialized systems, whereas for the U-initialization the headgroup ordering was not found in none of the cases. An experimentally proposed orthogonal unit cell with unit vector lengths of 5.52 and 8.42 $\AA$ [64] was used for the crystalline structure in the preset islands. Such a preset island was surrounded by a pre-equilibrated single layer of laying-down thiols. In the case of $N_{m}=953$ the stripes of standing-up surfactants (with the structure identical to the one in the islands) were initially preset from both sides of liquid $\mathrm{Hg}$. The rest of notations follow the ones in Tab. A.1. 


\begin{tabular}{l|l|r|r|c|l}
$N_{m}$ & $\sigma\left(\mathrm{nm}^{-2}\right)$ & $t_{e q}(\mathrm{~ns})$ & $t_{s}(\mathrm{~ns})$ & $\begin{array}{l}\text { Initial } \\
\text { config. }\end{array}$ & $\begin{array}{l}\text { Final } \\
\text { configurations }\end{array}$ \\
\hline \hline 240 & 0.53124 & 20.00 & 70.92 & $\mathrm{U}$ & PF of 1st LD thiol layer \\
\hline 378 & 0.83671 & 10.00 & 80.44 & $\mathrm{U}$ & PF of 1st LD thiol layer \\
\hline 650 & 1.43879 & 66.00 & 381.76 & $\mathrm{~T}$ & cr. SU islands from both sides \\
\hline 728 & 1.61144 & 54.00 & 260.56 & $\mathrm{~T}$ & cr. SU islands from both sides \\
\hline 906 & 2.00545 & 22.00 & 233.98 & $\mathrm{P}$ & cr. SU islands from both sides \\
\hline
\end{tabular}

Table A.5: Dodecanethiol (SC12) systems for the overall system cross-section of $29.808 \times 30.312=903.5 \mathrm{~nm}^{2} . N_{H g}=285670$. T stands for the initial surfactant configurations obtained by simply trimming the longer molecules (SC18) in the respective equilibrated conformations. The rest of the notations follow the ones in Tabs. A.1 and A.4. 


\section{Bibliography}

[1] J. A. Pelesko, Self Assembly: The Science of Things That Put Themselves Together (Chapman and Hall/CRC, Boca Raton, 2007).

[2] Y. S. Lee, Self-Assembly And Nanotechnology: A Force Balance Approach (Wiley, Hoboken, New Jersey, 2008).

[3] W. Lin, A. J. Clark, and F. Paesani, Langmuir 31, 2147 (2015).

[4] V. M. Kaganer, H. Möhlwald, and P. Dutta, Rev. Mod. Phys. 71, 779 (1999).

[5] H. Kraack, B. M. Ocko, P. S. Pershan, L. Tamam, and M. Deutsch, J. Chem. Phys. 121, 8003 (2004).

[6] J. C. Love, L. A. Estroff, J. K. Kriebel, R. G. Nuzzo, and G. M. Whitesides, Chem. Rev. 105, 1103 (2005).

[7] F. Schreiber, J. Phys.: Condens. Matter 16, R881 (2004).

[8] F. Schreiber, Prog. Surf. Sci. 65, 151 (2000).

[9] D. K. Schwartz, Annu. Rev. Phys. Chem. 52, 107 (2001).

[10] K. S.-Kunstman, P. Cyganic, B. Schüpbach, and A. Terfort, Phys. Chem. Chem. Phys. 12, 4400 (2010).

[11] T. Zhang, Z. Cheng, Y. Wang, Z. Li, C. Wang, Y. Li, and Y. Fang, Nano Lett. 10, 4738 (2010).

[12] O. Voznyy and J. J. Dubowski, Langmuir 24, 13299 (2008). 
[13] S. W. Han, S. J. Lee, and K. Kim, Langmuir 17, 6981 (2001).

[14] A. R. Noble-Luginbuhl and R. G. Nuzzo, Langmuir 17, 3937 (2001).

[15] P. E. Laibinis, G. M. Whitesides, D. L. Allara, Y.-T. Tao, A. N. Parikh, and R. G. Nuzzo, J. Am. Chem. Soc. 113, 7152 (1991).

[16] T.-C. G. N. Preparation and E. for Quantum Size Effects, Chem. Mater. 26, 2138 (2014).

[17] T. M. Uehara, H. B. de Aguiar, K. Bergamaski, and P. B. Miranda, J. Phys. Chem. C 118, 20374 (2014).

[18] C. Vericat, M. E. Vela, and R. C. Salvarezza, Phys. Chem. Chem. Phys. 7, 3258 (2005).

[19] L. L. Rouhana, M. D. Moussallem, and J. B. Schlenoff, J. Am. Chem. Soc. 133, 16080 (2011).

[20] S. Watcharinyanon, E. Moons, and L. S. O. Johansson, J. Phys. Chem. C 113, 1972 (2009).

[21] S.-S. Li, L.-P. Xu, L.-J. Wan, S.-T. Wang, and L. Jiang, J. Phys. Chem. B 110, 1794 (2006).

[22] P. Fenter, P. Eisenberger, and K. S. Liang, Phys. Rev. Lett. 70, 2447 (1993).

[23] R. G. Nuzzo, E. M. Korenic, and L. H. Dubois, J. Chem. Phys. 93, 767 (1990).

[24] N. Camillone III, C. E. D. Chidsey, G.-Y. Liu, and G. Scoles, J. Chem. Phys. 98, 3503 (1993).

[25] G. E. Poirier and E. D. Pylant, Science 272, 1145 (1996).

[26] J. Scherer, M. R. Vogt, O. M. Magnussen, and R. J. Behn, Langmuir 13, 7045 (1997).

[27] B. Haimov, A. Iakovlev, R. Glick-Carmi, L. Bloch, B. Rich, M. Müller, and B. Pokroy, J. Am. Chem. Soc. 138, 2585 (2016). 
[28] J. M. Devi, Prog. Nat. Sci. Mat. Int. 24, 405 (2014).

[29] L. Ramin and A. Jabbarzadeh, Langmuir 28, 4102 (2012).

[30] L. Ramin and A. Jabbarzadeh, J. Chem. Phys. 137, 174706 (2012).

[31] L. Ramin and A. Jabbarzadeh, Modelling Simul. Mater. Sci. Eng. 20, 085010 (2012).

[32] G. S. Longo, S. Kr. Bhattacharya, and S. Scandolo, J. Phys. Chem. C 116, 14883 (2012).

[33] A. Jiménez, A. Sarsa, M. Blázquez, and T. Pineda, J. Phys. Chem. C 114, 21309 (2010).

[34] Y. Ahn, J. K. Saha, G. C. Schatz, and J. Jang, J. Phys. Chem. C 115, 10668 (2011).

[35] P. K. Ghorai and S. C. Glotzer, J. Phys. Chem. C 111, 15857 (2007).

[36] O. Alexiadis, V. A. Harmandaris, V. G. Mavrantzas, and L. D. Site, J. Phys. Chem. C 111, 6380 (2007).

[37] B. J. Henz and M. R. Zachairah, in Proceedings of the HPCMP Users Group Conferense, edited by D. E. Post (Washington, DC, 2007), pp. 185-193.

[38] S. Vemparala and B. B. Karki, J. Chem. Phys. 121, 4323 (2004).

[39] B. Rai, S. P. Chetan, C. P. Malhotra, and K. G. Ayappa, Langmuir 20, 3138 (2004).

[40] L. Zhang, W. A. Goddard III, and S. Jiang, J. Chem. Phys. 117, 7342 (2002).

[41] W. D. Luedtke and U. Landman, J. Phys. Chem. B 102, 6566 (1998).

[42] R. Bhatai and B. J. Garrison, Langmuir 13, 4038 (1997).

[43] R. Bhatai and B. J. Garrison, Langmuir 13, 765 (1997).

[44] K. J. Tupper, R. J. Colton, and D. W. Brenner, Langmuir 10, 2041 (1994). 
[45] W. Mar and M. L. Klein, Langmuir 10, 188 (1994).

[46] J. Hautman and M. L. Klein, J. Chem. Phys. 91, 4994 (1989).

[47] T. Yi and S. Lichter, Phys. Rev. E 89, 062404 (2014).

[48] T. Miura and K. Seki, Phys. Rev. E 91, 052604 (2015).

[49] T. Miura and M. Mikami, Phys. Rev. E 81, 021801 (2010).

[50] E. A. Weiss, J. K. Kriebel, M.-A. Rampi, and G. M. Whitesides, Philos. Trans. R. Soc. A 365, 1509 (2007).

[51] L. Zhu, R. T. W. Popoff, and H.-Z. Yu, J. Phys. Chem. C 119, 1826 (2015).

[52] C. B. Babayco, P. Chang, D. P. Land, R. A. Kiehl, and A. N. Parikh, Langmuir 29, 8203 (2013).

[53] O. Seitz, M. Dai, F. S. Aguirre-Tostado, R. M. Wallace, and Y. J. Chabal, J. Am. Chem. Soc. 131, 18159 (2009).

[54] R. A. Kiehl, J. D. Le, P. Candra, R. C. Hoye, and T. R. Hoye, Appl. Phys. Lett. 88, 172102 (2006).

[55] A. Nitzan and M. A. Ratner, Science 300, 1384 (2003).

[56] D. M. Ruthven, Principles of Adsorption and Adsorption Processes (John Wiley \& Sons, New York, 1984).

[57] M. Doi and S. F. Edwards, The Theory of Polymer Dynamics (Clarendon Press, Oxford, 1994).

[58] O. Dannenberger, M. Buck, and M. Grunze, J. Phys. Chem. B 103, 2202 (1999).

[59] J. R. Rahn and R. B. Halloc, Langmuir 11, 650 (1995).

[60] W. L. Jorgensen, J. D. Madura, and C. J. Swenson, J. Am. Chem. Soc. 106, 6638 (1984).

[61] O. M. Magnussen, B. M. Ocko, M. Deutsch, M. L. Regan, P. S. Pershan, D. Abernathy, G. Grübel, and J.-F. Legrand, Nature 384, 250 (1996). 
[62] M. Deutsch, O. M. Magnussen, B. M. Ocko, and M. J. Regan, in Thin Films, edited by A. Ulman (Academic Press, San Diego, 1998), vol. 24, pp. 179-203.

[63] R. H. Petrucci, F. G. Herring, and J. D. M. C. Bissonnette, General Chemistry Principles and Modern Applications (Pearson Canada, Toronto, 2011), 10th ed.

[64] H. Kraack, L. Tamam, E. Sloutskin, M. Deutsch, and B. M. Ocko, Langmuir 23, 7571 (2007).

[65] B. M. Ocko, H. Kraack, P. S. Pershan, E. Sloutskin, L. Tamam, and M. Deutsch, Phys. Rev. Lett. 94, 017802 (2005).

[66] H. Kraack, B. M. Ocko, P. S. Pershan, E. Sloutskin, L. Tamam, and M. Deutsch, Langmuir 20, 5386 (2004).

[67] H. Kraack, B. M. Ocko, P. S. Pershan, E. Sloutskin, L. Tamam, and M. Deutsch, Langmuir 20, 5375 (2004).

[68] N. Collazo, S. Shin, and S. A. Rice, J. Chem. Phys. 96, 4735 (1992).

[69] M. E. Schmidt, S. Shin, and S. A. Rice, J. Chem. Phys. 104, 2114 (1996).

[70] M. P. Allen and D. J. Tildesley, Computer Simulation of Liquids (Clarendon Press, Oxford, 1991).

[71] M. E. Tuckerman, J. Alejandre, R. López-Renón, A. L. Jochim, and G. J. Martyna, J. Phys. A: Math. Gen. 39, 5629 (2006).

[72] G. Ciccotti and G. Kalibaeva, in Novel Methods in Soft Matter Simulations, edited by M. Karttunen, I. Vattulainen, and A. Lukkarinen (Springer-Verlag, Berlin Heidelberg, 2004), Lecture Notes in Physics, pp. 150-189.

[73] G. J. Martyna, M. E. Tuckerman, D. J. Tobias, and M. L. Klein, Mol. Phys. 87, 1117 (1996).

[74] D. Frenkel and B. Smit, Understanding Molecular Simulation (Academic Press, San Diego, California, USA, 2002). 
[75] L. D. Landau and E. M. Lifshitz, Statistical Physics, 1 (BPC Wheatons, Exeter, 1994), 3rd ed.

[76] H. C. Andersen, J. Chem. Phys. 72, 2384 (1979).

[77] S. Plimpton, J. Comp. Phys. 117, 1 (1995), URL http://lammps.sandia.gov.

[78] H. J. C. Berendsen, J. P. M. Postma, W. F. van Gunsteren, A. DiNola, and J. R. Haak, J. Chem. Phys. 81, 3684 (1984).

[79] B. L. C. Matthews, Molecular Dynamics with Deterministic and Stochastic Numerical Methods (Springer, Switzerland, 2015).

[80] G. J. Martyna, M. L. Klein, and M. Tuckerman, J. Chem. Phys. 97, 2635 (1992).

[81] G. J. Martyna, D. J. Tobias, and M. L. Klein, J. Chem. Phys. 101, 4177 (1994).

[82] W. Shinoda, M. Shiga, and M. Mikami, Phys. Rev. B 69, 134103 (2004).

[83] M. Tuckerman, B. J. Berne, and G. J. Martyna, J. Chem. Phys. 97, 1990 (1992).

[84] D. P. Landau and K. Binder, A Guide to Monte Carlo Simulations in Statistical Physics (Cambridge University Press, New York, 2005), 2nd ed.

[85] A. Chatterjee and D. G. Vlachos, J. Computer-Aided Mater. Des. 14, 253 (2007).

[86] C. W. Gardiner, Handbook of Stochastic Methods for Physics, Chemistry and the Natural Sciences (Springer-Verlag, Berlin Heidelberg, 1997), 2nd ed.

[87] N. Metropolis, A. W. Rosenbluth, M. N. Rosenbluth, and A. H. Teller, J. Chem. Phys. 21, 1087 (1953).

[88] D. Frenkel, P. Bladon, P. Bolhuis, and M. Hagen, Mol. Sim. 16, 127 (1996).

[89] A. Iakovlev, D. Bedrov, and M. Müller, Eur. Phys. J. B 88, 323 (2015).

[90] D. Chandler, J. D. Weeks, and H. C. Andersen, Science 220, 787 (1983).

[91] J. D. Weeks, D. Chandler, and H. C. Andersen, J. Chem. Phys. 54, 5237 (1971).

[92] N. F. Carnahan and K. E. Starling, J. Chem. Phys. 51, 635 (1969). 
[93] P. Attard, Thermodynamics and Statistical Mechanics: Equilibrium by Entropy Maximisation (Academic Press, London, 2002).

[94] J. A. Barker and D. Henderson, J. Chem. Phys. 47, 4714 (1967).

[95] J. Chang and S. I. Sandler, Mol. Phys. 81, 735 (1994).

[96] J. W. Cahn and J. E. Hilliard, J. Chem. Phys. 28, 258 (1958).

[97] S. V. Gupta, Practical Density Measurement and Hydrometry (IOP, Bristol and Philadelphia, 2002).

[98] O. M. Magnussen, B. M. Ocko, M. L. Regan, K. Penanen, P. S. Pershan, and M. Deutsch, Phys. Rev. Lett. 74, 4444 (1995).

[99] A. W. Adamson and A. P. Gast, Physical Chemistry of Surfaces (John Wiley \& Sons, New York, 1997), 6th ed.

[100] J. B. Ketterson and S. N. Song, Superconductivity (Cambridge University Press, Cambridge, 1999).

[101] P. Schwerdtfeger, R. Wesendrup, G. E. Moyano, A. J. Sadlej, J. Greif, and F. Hensel, J. Chem. Phys. 115, 7401 (2001).

[102] B. Paulus and K. Rosciszewski, Chem. Phys. Lett. 394, 96 (2004).

[103] Y.-P. Zhao and C.-C. Chen, Rev. Adhesion Adhesives 1, 114 (2013).

[104] A. Kutana and K. P. Giapis, Phys. Rev. B 76, 195444 (2007).

[105] A. Kutana and K. P. Giapis, Nano Lett 6, 656 (2006).

[106] A. Kutana and K. P. Giapis, Science 310, 1480 (2005).

[107] K. Saarnio, A. Frey, J. V. Niemi, H. Timonen, T. Rönkkö, P. Karjalainen, M. Vestenius, K. Teinilä, L. Pirjola, V. Niemelä, et al., J. Aerosol Sci. 73, 14 (2014).

[108] D. C. Kulshreshtha, Basic Electrical Engineering (Tata McGraw-Hill, New Delhi, 2009). 
[109] R. Eisler, Mercury Hazards to Living Organisms (CRC Press, Boca Raton, 2006).

[110] T. W. Clarkson, J. Trace Elem. Exp. Med. 11, 303 (1998).

[111] F. Zahir, S. J. Rizwi, S. K. Haq, and R. H. Khan, Environ. Toxicol. Phar. 20, 351 (2005).

[112] S. Tao, C. Li, X. Fan, G. Zeng, P. Lu, X. Zhang, Q. Wen, W. Zhao, D. Luo, and C. Fan, Chem. Eng. J. 210, 547 (2012).

[113] Z. Tan, L. Sun, J. Xiang, H. Zeng, Z. Liu, S. Hu, and J. Qiu, Carbon 50, 362 (2012)

[114] H.-R. Chen, C.-C. Chen, A. Satyanarayana Reddy, C.-Y. Chen, W. R. Li, M.-J. Tseng, H.-T. Liu, W. Pan, J. P. Maity, and S. B. Atla, Int. J. Mol. Sci. 12, 8245 (2011).

[115] B. Pokroy, B. Aichmayer, A. S. Schenk, B. Haimov, S. H. Fratzl, and J. Aizenberg, J. Am. Chem. Soc. 132, 14355 (2010).

[116] K. Hoshino, S. Tanaka, and F. Shimojo, J. Non-Cryst. Sol. 353, 3389 (2007).

[117] S. Munejiri, F. Shimojo, and K. Hoshino, J. Phys.: Condens. Matter 10, 4963 (1998).

[118] G. Tóth, J. Chem. Phys. 118, 3949 (2003).

[119] G. Raabe, B. D. Todd, and R. L. Sadus, J. Chem. Phys. 123, 034511 (2005).

[120] G. Raabe and R. J. Sadus, J. Chem. Phys. 119, 6691 (2003).

[121] J.-M. Bomont and J.-L. Bretonnet, J. Chem. Phys, 124, 054504 (2006).

[122] J.-M. Bomont and J.-L. Bretonnet, J. Phys.: Conf. Ser. 98, 042018 (2008).

[123] D. S. Chekmarev, M. Zhao, and S. A. Rice, Phys. Rev. E 59, 479 (1998).

[124] M. P. D’Evelyn and S. A. Rice, J. Chem. Phys. 78, 5081 (1983).

[125] L. Calderín, L. E. González, and D. J. González, Phys. Rev. B 87, 014201 (2013). 
[126] J.-M. Bomont, J.-L. Bretonnet, D. J. Gonzalez, and L. E. Gonzalez, Phys. Rev. B 79, 144202 (2009).

[127] M. H. Ghatee and M. Bahadori, J. Phys. Chem. B 108, 4141 (2004).

[128] H. Okumura and F. Yonezawa, J. Non-Cryst. Sol. 312-314, 260 (2002).

[129] S. Nagel, R. Redmer, and G. Röpke, J. Non-Cryst. Sol. 205-207, 247 (1996).

[130] E. Chacón, M. Reinaldo-Falagán, E. Velasco, and P. Tarazona, Phys. Rev. Lett. 87, 166101 (2001).

[131] D. K. Belashchenko, High Temperature 51, 40 (2013).

[132] D. K. Belashchenko, High Temperature 51, 878 (2013).

[133] D. K. Belashchenko, High Temperature 44, 675 (2006).

[134] A. A. Louis, J. Phys.: Condens. Matter 14, 9187 (2002).

[135] M. J. P. Nijmeijer, A. F. Bakker, C. Bruin, and J. H. Sikkenk, J. Chem. Phys. 89, 3789 (1988).

[136] J. K. Johnson, J. A. Zollweg, and K. E. Gubbins, Mol. Phys. 3, 591 (1993).

[137] K. S. Shen, R. D. Mountaim, and J. R. Errington, J. Phys. Chem. B 111, 6198 (2007).

[138] W. Kohn and L. J. Sham, Phys. Rev. 140, A1133 (1965).

[139] D. C. Rapaport, The Art of Molecular Dynamics Simulation (Cambridge University Press, Cambridge, 2005).

[140] M. S. Daw and M. I. Baskes, Phys. Rev. Lett. 50, 1285 (1983).

[141] M. S. Daw and M. I. Baskes, Phys. Rev. B 29, 6443 (1984).

[142] S. M. Foiles, Phys. Rev. B 32, 3409 (1985).

[143] S. M. Foiles and J. B. Adams, Phys. Rev. B 40, 5909 (1989). 
[144] L. M. Holzman, J. B. Adams, S. M. Foiles, and W. N. G. Hitchon, J. Mater. Res. 6, 298 (1991).

[145] K. Tamura and S. Hosokawa, Phys. Rev. B 58, 9030 (1998).

[146] J. G. Kirkwood and F. P. Buff, J. Chem. Phys. 17, 338 (1949).

[147] A. Trokhymchuk and J. Alejandre, J. Chem. Phys. 111, 8510 (1999).

[148] J.-P. Hansen and I. R. McDonald, Theory of Simple Liquids (Academic Press, London, 2006), 3rd ed.

[149] J. S. Rowlinson and B. Widom, Molecular Theory of Capillaryty (Oxford University Press, New York, 1984).

[150] A. V. Shevade, J. Zhou, M. T. Zin, and S. Jiang, Langmuir 17, 7566 (2001).

[151] T. B. Douglas, A. F. Ball, and D. C. Ginnings, J. Res. Natl. Bur. Stand. 46, 334 (1951).

[152] J. Wang and T. Hou, J. Chem. Theory Comput. 7, 2151 (2011).

[153] S. Ayrinhac, L. E. Bove, M. Morand, G. Le Marchand, F. Bergame, J. Philippe, and F. Decremps, J. Chem Phys. 140, 244201 (2014).

[154] B.-J. Lee, J.-H. Shim, and M. I. Baskes, Phys. Rev. B 68, 144112 (2003).

[155] J. Jalkanen and M. H. Müser, Model. Simul. Mater. Sc. Eng. 23, 074001 (2015).

[156] A. Iakovlev, D. Bedrov, and M. Müller, J. Phys. Chem. Lett. 7, 1546 (2016).

[157] M. A. Amat and G. C. Rutledge, J. Chem. Phys. 132, 114704 (2010).

[158] J.-F. Fuchs, H. Nedev, D. Poger, M. Ferrand, V. Brenner, J.-P. Dognon, and S. Crouzy, J. Comp. Chem. 27, 837 (2006).

[159] F. A. Escobedo and Z. Chen, J. Chem. Phys. 121, 11463 (2004).

[160] L. Ramin and A. Jabbarzadeh, Langmuir 27, 9748 (2011).

[161] A. D. Dussaud and M. Vignes-Adler, MRS Proceedings 464, 287 (1996). 
[162] H. Dobbs, Langmuir 15, 2586 (1999).

[163] L. G. MacDowell, P. Virnau, M. Müller, and K. Binder, J. Chem. Phys. 120, 5293 (2004).

[164] G. L. Lianf, D. W. N. B. G. Sumpter, and B. Wunderlich, J. Phys. Chem. 98, 11739 (1994).

[165] H. Lu, D. Zeysing, M. Kind, A. Terfort, and M. Zharnikov, J. Phys. Chem. C 117, 18967 (2013).

[166] R. Aveyard, J. H. Clint, D. Nees, and N. Quirke, Langmuir 16, 8820 (2000).

[167] L. Pocivavsek, S. L. Frey, K. Krishan, K. Gavrilov, P. Ruchala, A. J. Waring, F. J. Walther, M. Dennin, T. A. Witten, and K. Y. C. Lee, Soft Matter 4, 2019 (2008).

[168] H. Heinz and H. Ramezani-Dakhel, Chem. Soc. Rev. 45, 412 (2016). 


\section{List of publications}

1. A. Iakovlev, D. Bedrov, M. Müller, "Alkyl-Based Surfactants at a Liquid Mercury Surface: Computer Simulation of Structure, SelfAssembly, and Phase Behavior", J. Phys. Chem. Lett. 7, 1546-1553 (2016)

2. B. Haimov, A. Iakovlev, R. Glick-Carmi, L. Bloch, B. B. Rich, M. Müller, B. Pokroy, "Kinetics of Nanoscale Self-Assembly Measured on Liquid Drops by Macroscopic Optical Tensiometry: From Mercury to Water and Fluorocarbons", J. Am. Chem. Soc. 138, 2585-2591 (2016)

3. A. Iakovlev, D. Bedrov, M. Müller, "Surface tension of liquid mercury: a comparison of density-dependent and density-independent force fields", Eur. Phys. J. B 88, 323 (2015) 